ESAIM: M2AN 55 (2021) 2849-2897

https://doi.org/10.1051/m2an/2021063
ESAIM: Mathematical Modelling and Numerical Analysis

www.esaim-m2an.org

\title{
GLOBAL EXISTENCE OF WEAK SOLUTIONS TO UNSATURATED POROELASTICITY
}

\author{
Jakub Wiktor Both ${ }^{1} \odot$, IUliu Sorin Pop $^{2} \odot$ And IVAn Yotov ${ }^{3}$
}

\begin{abstract}
We study unsaturated poroelasticity, i.e., coupled hydro-mechanical processes in variably saturated porous media, here modeled by a non-linear extension of Biot's well-known quasi-static consolidation model. The coupled elliptic-parabolic system of partial differential equations is a simplified version of the general model for multi-phase flow in deformable porous media, obtained under similar assumptions as usually considered for Richards' equation. In this work, existence of weak solutions is established in several steps involving a numerical approximation of the problem using a physicallymotivated regularization and a finite element/finite volume discretization. Eventually, solvability of the original problem is proved by a combination of the Rothe and Galerkin methods, and further compactness arguments. This approach in particular provides the convergence of the numerical discretization to a regularized model for unsaturated poroelasticity. The final existence result holds under non-degeneracy conditions and natural continuity properties for the constitutive relations. The assumptions are demonstrated to be reasonable in view of geotechnical applications.
\end{abstract}

Mathematics Subject Classification. 35K61, 65M12, 74F10, 76S05.

Received August 4, 2020. Accepted October 2, 2021.

\section{INTRODUCTION}

Strongly coupled hydro-mechanical processes in porous media are occurring in various applications of societal relevance within, e.g., geotechnical, structural, and biomechanical engineering. Examples for instance are soil subsidence due to groundwater withdrawal, induced seismicity in geothermal reservoirs, swelling and drying shrinkage of concrete, and deformation of soft biological tissue components, to mention a few.

In the field of porous media, such microscopically complex processes are typically modeled by a continuum mechanics approach [17]. The multi-phasic solid-fluid mixture is considered a homogenized continuum, and both geometry, skeleton, and fluid properties are averaged over representative elementary volumes, consisting of a mixture of solid and fluid particles. Ultimately, the interaction of the different microscopic constituents is described by macroscopic effective equations.

Keywords and phrases. Poroelasticity, Biot model, variably saturated porous media, Richards' equation, existence of solutions; convergence of mesh-based discretization.

1 University of Bergen, Department of Mathematics, Allégaten 41, 5007 Bergen, Norway.

2 Hasselt University, Faculty of Sciences, Campus Diepenbeek, Agoralaan Gebouw D, 3590 Diepenbeek, Belgium.

3 University of Pittsburgh, Department of Mathematics, 301 Thackeray Hall, Pittsburgh, PA 15260, USA.

*Corresponding author: jakub.both@uib.no

(C) The authors. Published by EDP Sciences, SMAI 2021

This is an Open Access article distributed under the terms of the Creative Commons Attribution License (https://creativecommons.org/licenses/by/4.0), which permits unrestricted use, distribution, and reproduction in any medium, provided the original work is properly cited. 
The simplest macroscopic model accounting for the two-way coupling of single-phase flow and elastic deformation in a porous medium is Biot's linear quasi-static consolidation model. Its phenomenological derivation dates back to the seminal works by Terzaghi, [41] and Biot [4]. In the course of the last century, many more advanced models have been developed, accounting, e.g., for the presence of different interacting fluids, thermal effects, or chemical reactions [16,23].

In this paper, we consider a non-linear coupled system of partial differential equations, modeling the quasistatic consolidation of variably saturated porous media - also called unsaturated poroelasticity. The model is in particular relevant in soil mechanics. It can be obtained by simplifying the more general model for two-phase flow in deformable porous media, founded on macroscopic momentum and mass balances combined with constitutive relations [23] - it is assumed that one fluid phase can be simply neglected. This is a common practice for fluids with high viscosity ratios if the negligible fluid phase is continuous and connected to the atmosphere, i.e., the same hypotheses as for Richards' equation [30,39]. Finally, the resulting model generalizes Biot's consolidation model, now essentially non-linearly coupling Richards' equation and linear elasticity equations. It is highly nonlinear, potentially strongly coupled, and potentially degenerate, which makes its analysis challenging. In the fully saturated regime, it simplifies to the classical Biot equations.

Regarding the mathematical theory of poroelasticity, in particular Biot's linear quasi-static consolidation model has been well studied. Well-posedness including the existence, uniqueness, and regularity of solutions, has been established [3, 35, 47]. Lately, linear and non-linear extensions have become of increased interest. Well-posedness has been analyzed for the dynamic Biot-Allard system [24], Biot-Stokes systems [2,36], Biot's consolidation model with deformation dependent permeability [5,40], poroelasticity in fractured media modeled by phase-fields [26], general non-linear single-phase poroelasticity [42], poro-visco-elasticity [5,9], thermoporoelasticity [11,43], and further extensions modeled within a gradient flow framework [9], among others. In most problems, the coupling is linear.

Despite the large interest, rather few theoretical results have been established for unsaturated or multiphase poroelasticity. We highlight the results in [37] which constitute the first ever mathematical analysis of the consolidation of a variably saturated porous medium. In the aforementioned work, the existence of weak solutions is established under two strict model assumptions: (i) the coupling term in the fluid flow equation is linear; and (ii) after introducing a new pressure variable by applying the Kirchhoff transformation, the coupling and the diffusion terms in the mass balance equation simultaneously become linear. The second assumption implies a specific, artificial form of the so-called pore pressure, a non-linearity arising in the linear momentum balance. Ultimately, the result does not apply to the more general unsaturated poroelasticity model considered here. Moreover, the analysis accounts for non-linearly variable densities and porosities, and most importantly allows for degenerate situations.

On the other hand, the development of robust and efficient solution techniques for unsaturated or multiphase poroelasticity models is a very active research field; we mention $[8,12,46]$. So far, the development of discretization techniques and their analysis has mostly been limited to linear poroelasticity models; we merely mention [13], in which a stabilization technique for a finite element/finite volume discretization for multi-phase poroelasticity is numerically studied, and [7], in which convergence of the gradient discretization method is proved for two-phase flow in fractured, deformable media.

In this paper, the existence of weak solutions for the general model for unsaturated poroelasticity is established. The analysis, in essence, involves a variable transformation, a numerical approximation, and a discussion of the convergence of that approximation, using a priori estimates and compactness arguments, as pointed out in more detail in the following.

To simplify the analysis, the problem is first transformed utilizing the Kirchhoff transformation, a technique commonly used for the analysis of non-linear diffusion problems [1,37]. By this, the diffusion component of the mass balance becomes linear; for the considered model, a fully non-linear coupling and a non-linear storage coefficient remain present.

The subsequent analysis employs concepts of Rothe's method and the Galerkin method. A finite dimensional, numerical approximation is introduced by discretization in space and time, combining an implicit time stepping, 
a finite element method (FEM) for the mechanics equation, and a finite volume method involving a two-point flux approximation (TPFA) for the flow equation. The motivation for the chosen discretization is two-fold: (i) it is a common discretization in the field of poroelasticity, both in the research literature $[13,45]$ as well as in the industry; and important for this work, (ii) the specific choice of the discretization will allow for straightforward cancelling of the non-linear coupling terms, which will be crucial for deriving a priori estimates.

The analysis here relies on compactness arguments. Due to the nonlinear coupling terms, one needs a sufficient regularity of the solution, and appropriate a priori estimates. To this, prior to the discretization, a physicallymotivated regularization is introduced, accounting for the primary and secondary consolidation of variably saturated porous media with compressible grains [16]. From a mathematical point of view, the regularization transforms both balance equations into non-degenerate parabolic ones, and thereby naturally leads to higher regularity in time. Similar ideas have been used previously, e.g., for saturated poroelasticity $[5,22,28,35,42]$, and for unsaturated flow in porous media $[1,29,31,33]$.

In the remaining steps of the proof, a priori estimates are derived and compactness arguments are utilized in order to deduce, first, the convergence of the finite element/finite volume discretization to a (continuous) regularized solution for vanishing discretization parameters, and furthermore, convergence of the regularized solution to a weak solution of the transformed model for vanishing regularization parameters. Thereby, the existence of a weak solution to the transformed model will be proved. In the discussion of the limit of the spatial discretization specific finite volume techniques are utilized, inspired by $[19,21,34]$.

The present analysis requires an overall parabolic character of the coupled problem and natural continuity properties for the non-linearities. When passing the regularization to zero, those are ensured under specific material assumptions. The most important one requires the presence of a compressible fluid or solid grains, and a sufficiently stiff bulk. In the appendix, the assumptions are demonstrated to be satisfied for non-degenerating constitutive relations typically utilized in practical applications. Furthermore, focusing on the non-linear coupled character, some simplifying assumptions are made as the presence of an isotropic material, no gravity, and merely homogeneous essential along with inhomogeneous natural boundary conditions.

The rest of the paper is organized as follows. In Section 2, the model is introduced as derived in the engineering literature, and then transformed using the Kirchhoff transformation. In Section 3, the notion of a weak solution to the transformed problem is introduced, and the main result is stated: existence of a weak solution to the transformed problem under certain model assumptions and non-degeneracy conditions. The idea of the proof, consisting of six steps, is presented. The details of those six steps are the subject of the remaining Sections 4-9. In Section 10, a brief numerical test is provided illustrating the convergence of the used numerical scheme. In Appendix A, the feasibility of the required assumptions for the main result are discussed for widely used constitutive models from the literature. In addition, in Appendix B, technical results from the literature used in the proof of the main result are recalled for a comprehensive presentation.

\section{Mathematical model For unsaturated poroelasticity}

We consider a continuum mechanics model for unsaturated poroelasticity, a particular simplification of general multi-phase poroelasticity. As Biot's consolidation model, it is based on the fundamental principles of momentum and mass balance combined with constitutive relations. The model accounts for slight compressibility of the fluid as well as changing porosity due to compressible grains and volumetric deformation, as well pore pressure acting on the solid skeleton in the context of unsaturated media. It is valid under the assumptions of infinitesimal strains and the presence of two fluid phases, of which one is an active and the other is a passive phase; the displacement of the passive phase does not impede the advance of the active phase and can be neglected. Effectively, the model non-linearly couples the Richards equation and linear elasticity equations. For a detailed derivation, we refer to the textbooks $[16,23]$.

In the following, we recall the mathematical model employing the mechanical displacement and fluid pressure as primary variables. Additionally, the problem is transformed using the Kirchhoff transformation, a standard 
tool for the analysis of non-linear diffusion problems, cf., e.g., [1]. The resulting model will be subject of the subsequent analysis.

\subsection{The original mathematical model}

We consider a poroelastic medium occupying the open, connected, and bounded domain $\Omega \subset \mathbb{R}^{d}, d \in\{1,2,3\}$. Let $T>0$ denote the final time and $(0, T)$ denote the time interval of interest. Under the assumption of infinitesimal deformations of the skeleton, the poroelastic medium can be approximated as fixed in time. Let $Q_{T}:=\Omega \times(0, T)$ denote the space-time domain.

The momentum and mass balance equations as derived in [23] read on $Q_{T}$ :

$$
\begin{array}{r}
-\boldsymbol{\nabla} \cdot\left[2 \mu \boldsymbol{\varepsilon}(\boldsymbol{u})+\lambda \boldsymbol{\nabla} \cdot \boldsymbol{u} \mathbf{I}-\alpha p_{\text {pore }}\left(p_{\mathrm{w}}\right) \mathbf{I}\right]=\boldsymbol{f}, \\
\phi \partial_{t} s_{\mathrm{w}}\left(p_{\mathrm{w}}\right)+\phi c_{\mathrm{w}} s_{\mathrm{w}}\left(p_{\mathrm{w}}\right) \partial_{t} p_{\mathrm{w}}+\frac{1}{N} s_{\mathrm{w}}\left(p_{\mathrm{w}}\right) \partial_{t} p_{\text {pore }}\left(p_{\mathrm{w}}\right)+\alpha s_{\mathrm{w}}\left(p_{\mathrm{w}}\right) \partial_{t} \boldsymbol{\nabla} \cdot \boldsymbol{u}+\boldsymbol{\nabla} \cdot \boldsymbol{q}=h,
\end{array}
$$

where $\boldsymbol{u}$ and $p_{\mathrm{w}}$ are the primal variables, and denote the structural displacement and the fluid pressure (of the active phase), respectively. Furthermore, the volumetric flux $\boldsymbol{q}$ is described by the generalized Darcy law

$$
\boldsymbol{q}=-\kappa_{\mathrm{abs}} \kappa_{\mathrm{rel}}\left(s_{\mathrm{w}}\left(p_{\mathrm{w}}\right)\right)\left(\nabla p_{\mathrm{w}}-\rho_{\mathrm{w}} \boldsymbol{g}\right) .
$$

Moreover, in (2.1) and (2.2), $\mu$ and $\lambda$ denote Lamé parameters, $\boldsymbol{\varepsilon}(\boldsymbol{u})$ is the linearized strain tensor, $\alpha \in[0,1]$ is the Biot coefficient, $p_{\text {pore }}$ is an arbitrary pore pressure (in [23], the derivation is merely formulated using the so-called averaged pore pressure), $\boldsymbol{f}$ is an external volume force density; $\phi$ is the porosity, $s_{\mathrm{w}}$ is the fluid saturation, $c_{\mathrm{w}} \in[0, \infty)$ is the constant storage coefficient associated to fluid compressibility, $N \in(0, \infty]$ is the constant Biot modulus associated to the compressibility of solid grains, $\boldsymbol{q}$ is the volumetric flux, and $h$ is an external source term; $\kappa_{\mathrm{abs}}$ is a spatially varying absolute permeability, $\kappa_{\mathrm{rel}}$ is the relative permeability, $\rho_{\mathrm{w}}$ is the fluid density, and $\boldsymbol{g}$ is the gravitational acceleration. Typical ranges of values in geotechnical applications are $\mu, \lambda \sim 10^{7} \ldots 10^{11} \mathrm{~Pa}, \phi \sim 0.01 \ldots 0.7, c_{\mathrm{w}} \sim 10^{-11} \ldots 10^{-7} \mathrm{~Pa}^{-1}, N \sim 10^{10} \ldots 10^{12} \mathrm{~Pa}, \alpha \sim 0.05 \ldots 1$, $\kappa_{\text {abs }} \sim 10^{-21} \ldots 10^{-9} \mathrm{~m}^{2}$; see [16] for concrete examples.

To briefly explain the structure of (2.1), we note that the total stress in (2.1a) is defined via the Biot effective stress [16]; the first four terms in (2.1b) correspond to the weighted change in fluid mass $\rho_{\mathrm{w}}^{-1} \partial_{t}\left(\phi s_{\mathrm{w}} \rho_{\mathrm{w}}\right)$. They are obtained after applying the product rule, employing $\rho_{\mathrm{w}}^{-1} \partial_{t} \rho_{\mathrm{w}}=c_{\mathrm{w}} \partial_{t} p_{\mathrm{w}}$ for slightly compressible fluids, as well as the relation $\partial_{t} \phi=\alpha \partial_{t} \boldsymbol{\nabla} \cdot \boldsymbol{u}+\frac{1}{N} \partial_{t} p_{\text {pore }}$ for varying porosity [16,23].

Constitutive laws are assumed to be given for the fluid saturation $s_{\mathrm{w}}$, the relative permeability $\kappa_{\text {rel }}$, and the pore pressure $p_{\text {pore }}$. The medium is assumed homogeneous, aside from a heterogeneous matrix permeability. Consequently, the constitutive relationships remain the same everywhere. Commonly accepted in practice are the van Genuchten-Mualem relations $[27,44]$ with a non-decreasing saturation and a possibly degenerate relative permeability

$$
\begin{aligned}
s_{\mathrm{w}}\left(p_{\mathrm{w}}\right) & := \begin{cases}s_{\mathrm{w}, \mathrm{res}}+\left(1-s_{\mathrm{w}, \mathrm{res}}\right)\left[1+\left(-\alpha_{\mathrm{vG}} p_{\mathrm{w}}\right)^{n_{\mathrm{vG}}}\right]^{-m_{\mathrm{vG}},} & p_{\mathrm{w}} \leq 0, \\
1, & p_{\mathrm{w}} \geq 0,\end{cases} \\
s_{\mathrm{w}, \mathrm{eff}}\left(s_{\mathrm{w}}\right) & :=\frac{s_{\mathrm{w}}-s_{\mathrm{w}, \mathrm{res}}}{1-s_{\mathrm{w}, \mathrm{res}}}, \\
\kappa_{\mathrm{vG}}\left(s_{\mathrm{w}}\right) & :=\sqrt{s_{\mathrm{w}, \mathrm{eff}}\left(s_{\mathrm{w}}\right)}\left[1-\left(1-s_{\mathrm{w}, \mathrm{eff}}\left(s_{\mathrm{w}}\right)^{\frac{1}{m_{\mathrm{vG}}}}\right)^{m_{\mathrm{vG}}}\right]^{2}, \\
\kappa_{\mathrm{rel}}\left(s_{\mathrm{w}}\right) & := \begin{cases}\kappa_{\mathrm{vG}}\left(s_{\mathrm{w}, \mathrm{res}}+s_{\varepsilon}\right), & s_{\mathrm{w}}<s_{\mathrm{w}, \mathrm{res}}+s_{\varepsilon}, \\
\kappa_{\mathrm{vG}}\left(s_{\mathrm{w}}\right), & s_{\mathrm{w}} \geq s_{\mathrm{w}, \text { res }}+s_{\varepsilon},\end{cases}
\end{aligned}
$$

where $m_{\mathrm{vG}} \in(0,1), n_{\mathrm{vG}}=\left(1-m_{\mathrm{vG}}\right)^{-1}$, and $\alpha_{\mathrm{vG}}>0$ are model parameters, $s_{\mathrm{w}, \text { res }} \in[0,1)$ is the residual saturation, $s_{\mathrm{w}, \text { eff }}$ is the effective saturation, and $s_{\varepsilon} \in\left[0,1-s_{\mathrm{w}, \text { res }}\right)$ is a regularization parameter, ensuring 
non-degeneracy when chosen positive. In addition, we consider the widely-used equivalent pore pressure [16]

$$
p_{\text {pore }}\left(p_{\mathrm{w}}\right):=\int_{0}^{p_{\mathrm{w}}} s_{\mathrm{w}}(p) \mathrm{d} p .
$$

In the fully saturated regime, when $p_{\mathrm{w}} \geq 0$, the equivalent pore pressure reduces to the fluid pressure, which is consistent with poroelasticity models for fully saturated media. In the unsaturated regime, the pore pressure is equal to the volume averaged fluid pressure with a correction accounting for interfacial energy. As the fluid pressure equals the negative capillary pressure, it becomes negative in the unsaturated regime. We stress that although the subsequent analysis has these particular relations as examples, it does not depend on these particular choices for $s_{\mathrm{w}}, p_{\text {pore }}$ and $\kappa_{\text {rel }}$.

Under the hypothesis of small perturbations of the porosity, which are often applied along with the assumptions of linear elasticity [16], we can assume that the porosity $\phi$, acting as weight, is constant in time, equal to some reference porosity field $\phi_{0}$. With this, we also note that in the fully saturated regime, i.e., when $s_{\mathrm{w}} \equiv 1$ and, equivalently, $p_{\mathrm{w}} \geq 0$, the model equations reduce to the classical quasi-static Biot equations.

From now on, we consider a compact form of (2.1) and (2.2). Specifically, we seek $\left(\boldsymbol{u}, p_{\mathrm{w}}\right)$ such that on $Q_{T}$

$$
\begin{aligned}
-\boldsymbol{\nabla} \cdot\left[2 \mu \boldsymbol{\varepsilon}(\boldsymbol{u})+\lambda \boldsymbol{\nabla} \cdot \boldsymbol{u} \mathbf{I}-\alpha p_{\text {pore }}\left(p_{\mathrm{w}}\right) \mathbf{I}\right] & =\boldsymbol{f}, \\
\partial_{t} b\left(p_{\mathrm{w}}\right)+\alpha s_{\mathrm{w}}\left(p_{\mathrm{w}}\right) \partial_{t} \boldsymbol{\nabla} \cdot \boldsymbol{u}-\boldsymbol{\nabla} \cdot\left(\kappa_{\mathrm{abs}} \kappa_{\mathrm{rel}}\left(s_{\mathrm{w}}\left(p_{\mathrm{w}}\right)\right)\left(\boldsymbol{\nabla} p_{\mathrm{w}}-\rho_{\mathrm{w}} \boldsymbol{g}\right)\right) & =h,
\end{aligned}
$$

where in accordance to $(2.1 \mathrm{~b})$ the function $b$ is defined as

$$
b\left(p_{\mathrm{w}}\right)=\phi_{0} s_{\mathrm{w}}\left(p_{\mathrm{w}}\right)+c_{\mathrm{w}} \phi_{0} \int_{0}^{p_{\mathrm{w}}} s_{\mathrm{w}}(p) \mathrm{d} p+\frac{1}{N} \int_{0}^{p_{\mathrm{w}}} s_{\mathrm{w}}(p) p_{\text {pore }}^{\prime}(p) \mathrm{d} p .
$$

In order to close the system (2.5), we consider non-overlapping partitions $\left\{\Gamma_{\mathrm{D}}^{\mathrm{m}}, \Gamma_{\mathrm{N}}^{\mathrm{m}}\right\}$ and $\left\{\Gamma_{\mathrm{D}}^{\mathrm{f}}, \Gamma_{\mathrm{N}}^{\mathrm{f}}\right\}$ of the boundary $\partial \Omega$, with $\Gamma_{\mathrm{D}}^{\mathrm{m}} \cup \Gamma_{\mathrm{N}}^{\mathrm{m}}=\Gamma_{\mathrm{D}}^{\mathrm{f}} \cup \Gamma_{\mathrm{N}}^{\mathrm{f}}=\partial \Omega$, and $\Gamma_{\mathrm{D}}^{\mathrm{m}}$ as well as $\Gamma_{\mathrm{D}}^{\mathrm{f}}$ having positive measure. Then mixed boundary conditions are imposed,

$$
\begin{aligned}
\boldsymbol{u} & =\boldsymbol{u}_{\mathrm{D}} & & \text { on } \Gamma_{\mathrm{D}}^{\mathrm{m}} \times(0, T), \\
\left(2 \mu \boldsymbol{\varepsilon}(\boldsymbol{u})+\lambda \boldsymbol{\nabla} \cdot \boldsymbol{u} \mathbf{I}-\alpha p_{\text {pore }}\left(p_{\mathrm{w}}\right) \mathbf{I}\right) \boldsymbol{n} & =\boldsymbol{\sigma}_{\mathrm{N}} & & \text { on } \Gamma_{\mathrm{N}}^{\mathrm{m}} \times(0, T), \\
p_{\mathrm{w}} & =p_{\mathrm{w}, \mathrm{D}} & & \text { on } \Gamma_{\mathrm{D}}^{\mathrm{f}} \times(0, T), \\
-\kappa_{\text {abs }} \kappa_{\mathrm{rel}}\left(s_{\mathrm{w}}\left(p_{\mathrm{w}}\right)\right)\left(\boldsymbol{\nabla} p_{\mathrm{w}}-\rho_{\mathrm{w}} \boldsymbol{g}\right) \cdot \boldsymbol{n} & =w_{\mathrm{N}} & & \text { on } \Gamma_{\mathrm{N}}^{\mathrm{f}} \times(0, T) .
\end{aligned}
$$

Furthermore, the pressure satisfies the initial condition

$$
p_{\mathrm{w}}=p_{\mathrm{w}, 0}, \quad \text { in } \Omega \times\{0\} .
$$

Finally, for the initial displacement, $\boldsymbol{u}(0)$, we only consider compatible states, satisfying

$$
-\boldsymbol{\nabla} \cdot\left[2 \mu \boldsymbol{\varepsilon}(\boldsymbol{u}(0))+\lambda \boldsymbol{\nabla} \cdot \boldsymbol{u}(0) \mathbf{I}-\alpha p_{\text {pore }}\left(p_{\mathrm{w}, 0}\right) \mathbf{I}\right]=\boldsymbol{f}(0) .
$$

This also implicitly defines an initial condition for the volumetric deformation $\boldsymbol{\nabla} \cdot \boldsymbol{u}(0)$. Putting the focus on the non-linear and coupled character of the balance equations, in the subsequent, mathematical analysis, we consider a simplified setting, resulting in particular in a simpler notation. We neglect gravity and consider merely homogeneous essential boundary conditions, i.e., from now on we set $\boldsymbol{g} \equiv \mathbf{0}, \boldsymbol{u}_{\mathrm{D}} \equiv \mathbf{0}$ and $p_{\mathrm{w}, \mathrm{D}} \equiv 0$.

\subsection{The mathematical model under the Kirchhoff transformation}

The Kirchhoff transformation defines a new pressure-like variable

$$
\chi\left(p_{\mathrm{w}}\right)=\int_{0}^{p_{\mathrm{w}}} \kappa_{\text {rel }}\left(s_{\mathrm{w}}(\tilde{p})\right) \mathrm{d} \tilde{p} .
$$


Assuming the constitutive laws satisfy $\kappa_{\text {rel }}\left(s_{\mathrm{w}}(p)\right)>0$, for all $p \in \mathbb{R}$, the transformation (2.10) can be inverted. Given constitutive laws for $b, s_{\mathrm{w}}, \kappa_{\text {rel }}, p_{\text {pore }}$, as e.g., the equivalent pore pressure (2.4) and the van GenuchtenMualem relations (2.3), from now on, we consider the Kirchhoff transformation $\chi$ as unknown instead of $p_{\mathrm{w}}$, and redefine all functions accordingly

$$
\begin{aligned}
& \hat{p}_{\mathrm{w}}:=\chi^{-1}, \quad \hat{p}_{\text {pore }}:=p_{\text {pore }} \circ \chi^{-1}, \quad \hat{b}:=b \circ \chi^{-1}, \\
& \hat{s}_{\mathrm{w}}:=s_{\mathrm{w}} \circ \chi^{-1}, \quad \hat{\kappa}_{\text {rel }}:=\kappa_{\text {rel }} \circ \hat{s}_{\mathrm{w}} .
\end{aligned}
$$

Then under the assumption of a homogeneous relative permeability and saturation, the non-linear Biot equations (2.5)-(2.9) reduce to finding $(\boldsymbol{u}, \chi)$, satisfying

$$
\begin{aligned}
-\boldsymbol{\nabla} \cdot\left(2 \mu \boldsymbol{\varepsilon}(\boldsymbol{u})+\lambda \boldsymbol{\nabla} \cdot \boldsymbol{u I}-\alpha \hat{p}_{\text {pore }}(\chi) \mathbf{I}\right)=\boldsymbol{f} & \text { in } Q_{T}, \\
\partial_{t} \hat{b}(\chi)+\alpha \hat{s}_{\mathrm{w}}(\chi) \partial_{t} \boldsymbol{\nabla} \cdot \boldsymbol{u}-\boldsymbol{\nabla} \cdot\left(\kappa_{\mathrm{abs}} \boldsymbol{\nabla} \chi\right)=h & \text { in } Q_{T},
\end{aligned}
$$

subject to the adapted boundary conditions

$$
\begin{aligned}
\boldsymbol{u} & =\mathbf{0} & & \text { on } \Gamma_{\mathrm{D}}^{\mathrm{m}} \times(0, T), \\
\left(2 \mu \varepsilon(\boldsymbol{u})+\lambda \boldsymbol{\nabla} \cdot \boldsymbol{u} \mathbf{I}-\alpha \hat{p}_{\text {pore }}(\chi) \mathbf{I}\right) \boldsymbol{n} & =\boldsymbol{\sigma}_{\mathrm{N}} & & \text { on } \Gamma_{\mathrm{N}}^{\mathrm{m}} \times(0, T), \\
\chi & =0 & & \text { on } \Gamma_{\mathrm{D}}^{\mathrm{f}} \times(0, T), \\
-\kappa_{\text {abs }} \boldsymbol{\nabla} \chi \cdot \boldsymbol{n} & =w_{\mathrm{N}} & & \text { on } \Gamma_{\mathrm{N}}^{\mathrm{f}} \times(0, T),
\end{aligned}
$$

and the initial conditions

$$
\chi=\chi_{0}:=\chi\left(p_{\mathrm{w}, 0}\right) \quad \text { in } \Omega \times\{0\},
$$

with $\boldsymbol{u}(0)$ satisfying

$$
-\boldsymbol{\nabla} \cdot\left[2 \mu \boldsymbol{\varepsilon}(\boldsymbol{u}(0))+\lambda \boldsymbol{\nabla} \cdot \boldsymbol{u}(0) \mathbf{I}-\alpha p_{\mathrm{pore}}\left(p_{\mathrm{w}, 0}\right) \mathbf{I}\right]=\boldsymbol{f}(0)
$$

We highlight that after applying the Kirchhoff transformation, the transformed system (2.12) remains nonlinear and coupled. The main nonlinearities are $\hat{b}, \hat{p}_{\text {pore }}, \hat{s}_{\mathrm{w}}$. Yet, the diffusion term is now linear.

\section{Main RESUlt - EXISTEnCE OF A WEAK SOlution FOR The UnSATURATED POROELASTICITY MODEL}

The main result of this work is the existence of a weak solution for the unsaturated poroelasticity model under the Kirchhoff transformation, $c f$., Section 2.2. It should be noted, that the proof utilizes the convergence of a numerical approximation towards a weak solution and thereby also suggests a numerical scheme. The scheme involves the finite element and the finite volume method.

In the following, we state the main result. This includes the notion of a weak solution, required assumptions and the idea of the proof. The details of the proof are the subject of the remainder of this paper.

\subsection{Definition of a weak solution}

We use the standard notation for $L^{p}$, Sobolev $\left(H^{k}\right.$ and $\left.W^{k, p}\right)$ and Bochner spaces, together with their inherent norms and scalar products. Let $\langle\cdot, \cdot\rangle$ denote the duality pairing between a dual and its primal space; for $L^{2}(\Omega)$ this becomes the standard scalar product for scalars, vectors and tensors. Let

$$
\begin{aligned}
& \boldsymbol{V}:=\left\{\boldsymbol{v} \in H^{1}(\Omega)^{d} \mid \boldsymbol{v}_{\left.\right|_{\Gamma_{\mathrm{D}}^{\mathrm{m}}}}=\mathbf{0}\right\} \\
& Q:=\left\{q \in H^{1}(\Omega) \mid q_{\left.\right|_{\Gamma_{\mathrm{D}}^{\mathrm{f}}}}=0\right\}
\end{aligned}
$$


denote the function spaces corresponding to mechanical displacement and fluid pressure, respectively, incorporating essential boundary conditions. We abbreviate the bilinear form associated to linear elasticity

$$
a(\boldsymbol{u}, \boldsymbol{v}):=2 \mu \int_{\Omega} \boldsymbol{\varepsilon}(\boldsymbol{u}): \boldsymbol{\varepsilon}(\boldsymbol{u}) \mathrm{d} x+\lambda \int_{\Omega} \boldsymbol{\nabla} \cdot \boldsymbol{u} \boldsymbol{\nabla} \cdot \boldsymbol{v} \mathrm{d} x, \quad \boldsymbol{u}, \boldsymbol{v} \in \boldsymbol{V}
$$

and define $\|\cdot\|_{\boldsymbol{V}}:=a(\cdot, \cdot)^{1 / 2}$, which induces a norm on $\boldsymbol{V}$ due to Korn's inequality. Moreover, we combine the external body and surface sources as elements in $\boldsymbol{V}^{\star}$ and $Q^{\star}$, the duals of $\boldsymbol{V}$ and $Q$, respectively. Let $\boldsymbol{f}_{\text {ext }}=\left(\boldsymbol{f}, \boldsymbol{\sigma}_{\mathrm{N}}\right)$ and $h_{\mathrm{ext}}=\left(h, w_{\mathrm{N}}\right)$ be defined by

$$
\begin{aligned}
\left\langle\boldsymbol{f}_{\text {ext }}, \boldsymbol{v}\right\rangle: & =\int_{\Omega} \boldsymbol{f} \cdot \boldsymbol{v} \mathrm{d} x+\int_{\Gamma_{\mathrm{N}}^{\mathrm{m}}} \boldsymbol{\sigma}_{\mathrm{N}} \cdot \boldsymbol{v} \mathrm{d} s, & \boldsymbol{v} \in \boldsymbol{V}, \\
\left\langle h_{\text {ext }}, q\right\rangle:=\int_{\Omega} h q \mathrm{~d} x+\int_{\Gamma_{\mathrm{N}}^{\mathrm{f}}} w_{\mathrm{N}} q \mathrm{~d} s, & & q \in Q .
\end{aligned}
$$

Definition 3.1 (Weak solution). A weak solution to $(2.12)-(2.15)$ is a pair $(\boldsymbol{u}, \chi) \in L^{2}(0, T ; \boldsymbol{V}) \times L^{2}(0, T ; Q)$ satisfying the following (W1)-(W4):

(W1) $\hat{p}_{\text {pore }}(\chi) \in L^{2}\left(Q_{T}\right), \hat{s}_{\mathrm{w}}(\chi) \in L^{\infty}\left(Q_{T}\right)$.

(W2) $\hat{b}(\chi) \in L^{\infty}\left(0, T ; L^{1}(\Omega)\right)$ and $\partial_{t} \hat{b}(\chi) \in L^{2}\left(0, T ; Q^{\star}\right)$ such that

$$
\int_{0}^{T}\left\langle\partial_{t} \hat{b}(\chi), q\right\rangle \mathrm{d} t+\int_{0}^{T}\left\langle\hat{b}(\chi)-\hat{b}\left(\chi_{0}\right), \partial_{t} q\right\rangle \mathrm{d} t=0,
$$

for $q \in L^{2}(0, T ; Q) \cap W^{1,1}\left(0, T ; L^{\infty}(\Omega)\right)$ with $q(T)=0$.

(W3) $\partial_{t} \boldsymbol{\nabla} \cdot \boldsymbol{u} \in L^{2}\left(Q_{T}\right)$ such that

$$
\int_{0}^{T}\left\langle\partial_{t} \boldsymbol{\nabla} \cdot \boldsymbol{u}, q\right\rangle \mathrm{d} t+\int_{0}^{T}\left\langle\boldsymbol{\nabla} \cdot \boldsymbol{u}-\boldsymbol{\nabla} \cdot \boldsymbol{u}_{0}, \partial_{t} q\right\rangle \mathrm{d} t=0,
$$

for all $q \in H^{1}\left(0, T ; L^{2}(\Omega)\right)$ with $q(T)=0$, where for all $\boldsymbol{v} \in \boldsymbol{V}, \boldsymbol{u}_{0} \in \boldsymbol{V}$ satisfies

$$
a\left(\boldsymbol{u}_{0}, \boldsymbol{v}\right)-\alpha\left\langle\hat{p}_{\text {pore }}\left(\chi_{0}\right), \boldsymbol{\nabla} \cdot \boldsymbol{v}\right\rangle=\left\langle\boldsymbol{f}_{\text {ext }}(0), \boldsymbol{v}\right\rangle .
$$

(W4) $(\boldsymbol{u}, \chi)$ satisfies for all $(\boldsymbol{v}, q) \in L^{2}(0, T ; \boldsymbol{V}) \times L^{2}(0, T ; Q)$

$$
\begin{array}{r}
\int_{0}^{T}\left[a(\boldsymbol{u}, \boldsymbol{v})-\alpha\left\langle\hat{p}_{\text {pore }}(\chi), \boldsymbol{\nabla} \cdot \boldsymbol{v}\right\rangle\right] \mathrm{d} t=\int_{0}^{T}\left\langle\boldsymbol{f}_{\text {ext }}, \boldsymbol{v}\right\rangle \mathrm{d} t, \\
\int_{0}^{T}\left[\left\langle\partial_{t} \hat{b}(\chi)+\alpha \hat{s}_{\mathrm{w}}(\chi) \partial_{t} \boldsymbol{\nabla} \cdot \boldsymbol{u}, q\right\rangle+\left\langle\kappa_{\mathrm{abs}} \boldsymbol{\nabla} \chi, \nabla q\right\rangle\right] \mathrm{d} t=\int_{0}^{T}\left\langle h_{\text {ext }}, q\right\rangle \mathrm{d} t .
\end{array}
$$

Remark 3.2 (Discussion of (W1)). For the constitutive relations (2.3) and the equivalent pore pressure (2.4), (W1) is satisfied if, e.g., $\chi \in L^{2}\left(Q_{T}\right)$, as discussed in more detail in Appendix A.

Remark 3.3. We note that the weak formulation of the initial conditions (W3) of the volumetric deformation allows for a stronger formulation. See Lemma 9.6 for more information. In fact, more regularity is asked for in (W3) than required for $\hat{s}_{\mathrm{w}}(\chi) \partial_{t} \boldsymbol{\nabla} \cdot \boldsymbol{u} \in L^{2}\left(0, T ; Q^{\star}\right)$ to be well-defined; however, the higher regularity will be required for interpreting the initial data properly. 


\subsection{Assumptions on model and data}

For proving the existence of a weak solution, we require several assumptions on the model, including the constitutive laws, model parameters, and data. The assumptions on the constitutive laws read:

$(\mathrm{A} 0) s_{\mathrm{W}}: \mathbb{R} \rightarrow(0,1]$ and $\kappa_{\text {rel }}:[0,1] \rightarrow[0,1]$ are such that $\kappa_{\text {rel }}\left(s_{\mathrm{w}}(p)\right)>0$, for all $p \in \mathbb{R}$ allowing for defining $\hat{p}_{\mathrm{w}}, \hat{b}, \hat{s}_{\mathrm{w}}, \hat{p}_{\text {pore }}$, and $\hat{\kappa}_{\text {rel }}$ as in $(2.11)$.

(A1) $\hat{b}: \mathbb{R} \rightarrow \mathbb{R}$ is continuous and non-decreasing.

(A2) $\hat{s}_{\mathrm{w}}: \mathbb{R} \rightarrow(0,1]$ is continuous and differentiable a.e., and $\hat{s}_{\mathrm{w}}(\chi)=1$ for $\chi \geq 0$.

(A3) $\hat{p}_{\text {pore }}: \mathbb{R} \rightarrow \mathbb{R}$ is continuously differentiable, non-decreasing, and $\hat{p}_{\text {pore }}(\chi)=\chi$ for $\chi \geq 0$.

(A4) $\frac{\hat{p}_{\text {pore }}}{\hat{s}_{\mathrm{w}}}: \mathbb{R} \rightarrow \mathbb{R}$ is invertible and uniformly increasing, i.e., there exists a constant $c_{\hat{p}_{\text {pore }} / \hat{s}_{\mathrm{w}}}>0$ satisfying $\left(\frac{\hat{p}_{\text {pore }}}{\hat{s}_{\mathrm{w}}}\right)^{\prime}(\chi) \geq c_{\hat{p}_{\text {pore }} / \hat{s}_{\mathrm{w}}}$ for all $\chi \in \mathbb{R}$.

Assumptions (A0)-(A4) are valid for standard constitutive laws, as those presented in Section 2, cf., Appendix A for more details. The assumptions on the model parameters read:

(A5) $\mu>0, \lambda \geq 0, \alpha \geq 0$ are (for simplicity) constant, defining the bulk modulus $K_{\mathrm{dr}}:=\frac{2 \mu}{d}+\lambda>0$.

(A6) $\kappa_{\text {abs }}$ is continuous in $\Omega$ and uniformly bounded from below and above, such that there exist constants $0<\kappa_{\mathrm{m}, \mathrm{abs}} \leq \kappa_{\mathrm{M}, \mathrm{abs}}<\infty$ with $\kappa_{\mathrm{abs}} \in\left[\kappa_{\mathrm{m}, \mathrm{abs}}, \kappa_{\mathrm{M}, \mathrm{abs}}\right]$ in $\Omega$.

The assumptions on the external load and source terms read:

(A7) $\boldsymbol{f} \in H^{1}\left(0, T ; L^{2}(\Omega)^{d}\right), \sigma_{\mathrm{N}} \in H^{1}\left(0, T ; H^{-1 / 2}\left(\Gamma_{\mathrm{N}}^{\mathrm{m}}\right)\right), h \in H^{1}\left(0, T ; L^{2}(\Omega)\right), w_{\mathrm{N}} \in H^{-1 / 2}\left(\Gamma_{\mathrm{N}}^{\mathrm{f}}\right)$ such that $\boldsymbol{f}_{\text {ext }} \in H^{1}\left(0, T ; \boldsymbol{V}^{\star}\right)$ and $h_{\text {ext }} \in H^{1}\left(0, T ; Q^{\star}\right)$, where for $t \in(0, T)$

$$
\begin{aligned}
\left\|\boldsymbol{f}_{\text {ext }}(t)\right\|_{\boldsymbol{V}^{\star}} & :=\sup _{\mathbf{0} \neq \boldsymbol{v} \in \boldsymbol{V}} \frac{\left\langle\boldsymbol{f}_{\mathrm{ext}}(t), \boldsymbol{v}\right\rangle}{\|\boldsymbol{v}\|_{\boldsymbol{V}}}, \\
\left\|h_{\mathrm{ext}}(t)\right\|_{Q^{\star}} & :=\sup _{\mathbf{0} \neq q \in Q} \frac{\left\langle h_{\mathrm{ext}}(t), q\right\rangle}{\|q\|_{Q}},
\end{aligned}
$$

and analogously norms corresponding to Bochner spaces as $\left\|\boldsymbol{f}_{\text {ext }}\right\|_{H^{1}\left(0, T ; \boldsymbol{V}^{\star}\right)}$, etc.

The assumptions on the initial data read:

(A8) $\left(\boldsymbol{u}_{0}, \chi_{0}\right) \in \boldsymbol{V} \times Q$ is sufficiently regular such that there exists a constant $C_{0}$ satisfying

$$
\left.\left\|\boldsymbol{u}_{0}\right\|_{\boldsymbol{V}}^{2}+\left\|\boldsymbol{\nabla} \chi_{0}\right\|_{L^{2}(\Omega)}^{2}+\left\|\hat{b}\left(\chi_{0}\right)\right\|_{L^{1}(\Omega)}+\| \hat{B}\left(\chi_{0}\right)\right)\left\|_{L^{1}(\Omega)}+\right\| \bar{B}\left(\frac{\hat{p}_{\text {pore }}\left(\chi_{0}\right)}{\hat{s}_{\mathrm{w}}\left(\chi_{0}\right)}\right)\left\|_{L^{1}(\Omega)}+\right\| \hat{p}_{\text {pore }}\left(\chi_{0}\right) \|_{L^{2}(\Omega)}^{2} \leq C_{0},
$$

where $\hat{B}$ and $\bar{B}$ are energies defined as

$$
\begin{aligned}
& \hat{B}(z):=\int_{0}^{z}(\hat{b}(z)-\hat{b}(s)) \mathrm{d} s \geq 0, \\
& \bar{B}(z):=\int_{0}^{z}(\bar{b}(z)-\bar{b}(s)) \mathrm{d} s \geq 0 .
\end{aligned}
$$

Note, that (A1) implies the existence of a convex $C^{1}$-potential $\hat{\psi}: \mathbb{R} \rightarrow \mathbb{R}$, such that $\hat{b}=\hat{\psi}^{\prime}$. In this context, $\hat{B}$ can be related to the Legendre transform of $\xi \mapsto \hat{\psi}(\xi)-\hat{\psi}(0)$ composed with $\hat{b}$, [1] see also Lemma B.12 - similarly for $\bar{B}$.

Additionally, the following non-degeneracy conditions are assumed:

(ND1) There exists a constant $C_{\mathrm{ND}, 1}>0$ such that

$$
\left|\frac{\hat{p}_{\text {pore }}(\chi)}{\hat{s}_{\mathrm{w}}(\chi) \chi}\right| \leq C_{\mathrm{ND}, 1}, \quad \text { for all } \chi \in \mathbb{R} .
$$


(ND2) There exists a constant $C_{\mathrm{ND}, 2}>0$ such that

$$
C_{\mathrm{ND}, 2}^{-1} \leq \hat{p}_{\text {pore }}^{\prime}(\chi) \leq C_{\mathrm{ND}, 2}, \quad \text { for all } \chi \in \mathbb{R} .
$$

(ND3) The bulk modulus $K_{\mathrm{dr}}=\frac{2 \mu}{d}+\lambda$ is sufficiently large and satisfies

$$
K_{\mathrm{dr}} \geq \frac{\alpha^{2}}{2} \frac{\left(\hat{s}_{\mathrm{w}}(\chi)-\hat{p}_{\text {pore }}^{\prime}(\chi)\right)^{2}}{\hat{b}^{\prime}(\chi)}, \quad \text { for all } \chi \in \mathbb{R} .
$$

Physical interpretation of the non-degeneracy conditions. The condition (ND1) essentially means that the equivalent pore pressure essentially behaves like the transformed pressure. The condition (ND2) states that the pore pressure $\hat{p}_{\text {pore }}$ essentially behaves as the transformed pressure $\chi$; in Appendix A, (ND2) is showed to be satisfied for the equivalent pore pressure involving regularized hydraulic properties. Finally, the condition (ND3) essentially requires the mechanical system to be sufficiently stiff in relation to the system's effective compressibility governed by the compressibilities of the fluid and solid grains, as well as the hydraulic properties. In Appendix A, the conditional relation (ND3) is discussed for the constitutive laws presented in Section 2. To conclude, (ND3) can be expected to be satisfied in several practical situations.

\subsection{Existence of solutions for the unsaturated poroelasticity model}

This section is presenting the main result together with the main steps of the proof.

Theorem 3.4 (Existence of a weak solution to the unsaturated poroelasticity model). Under the model assumptions (A0)-(A8) and the non-degeneracy conditions (ND1)-(ND3), there exists a weak solution of (2.12)-(2.15) in the sense of Definition 3.1.

We observe that uniqueness is not addressed here. The fully coupled, and nonlinear character of the problem makes it in particular difficult to use any monotonicity arguments. This aspect is left open, for further research.

The main idea of the proof of Theorem 3.4 is to use the Galerkin method in combination with compactness arguments. The main difficulty here is the control over the non-linear coupling terms. For this a regularization approach is used. After all, the proof consists of six steps. In the following, we present the idea of each step. Details are subject of the remainder of the article and will be presented in the six, subsequent sections.

Step 1: physically meaningful regularization. Applying the Galerkin method along with compactness arguments for the original problem (3.2) is challenging due to the coupling terms. A simple way to control the term $\partial_{t} \boldsymbol{\nabla} \cdot \boldsymbol{u}$ is to add a suitable regularization term in the mechanics equation (3.2a). As the coupling terms also involve non-linearities in the Kirchhoff pressure, ultimately strong compactness is required. Therefore, we add a coercive term in the flow equation, which allows for controlling the term $\partial_{t} \chi$. In this way, one can control the coupling terms, and eventually leading to convergence.

From a physical point of view, the regularized model accounts for secondary consolidation and compressible solid grains. In mathematical terms, it reads as follows. For given regularization parameters $\zeta, \eta>0$, find $\left(\boldsymbol{u}_{\zeta \eta}, \chi_{\zeta \eta}\right)$ to be the solution to the variational equations

$$
\begin{array}{r}
\int_{0}^{T}\left[\zeta a\left(\partial_{t} \boldsymbol{u}_{\zeta \eta}, \boldsymbol{v}\right)+a\left(\boldsymbol{u}_{\zeta \eta}, \boldsymbol{v}\right)-\alpha\left\langle\hat{p}_{\text {pore }}\left(\chi_{\zeta \eta}\right), \boldsymbol{\nabla} \cdot \boldsymbol{v}\right\rangle\right] \mathrm{d} t=\int_{0}^{T}\left\langle\boldsymbol{f}_{\text {ext }}, \boldsymbol{v}\right\rangle \mathrm{d} t \\
\int_{0}^{T}\left[\left\langle\partial_{t} \hat{b}_{\eta}\left(\chi_{\zeta \eta}\right)+\alpha \hat{s}_{\mathrm{w}}\left(\chi_{\zeta \eta}\right) \partial_{t} \boldsymbol{\nabla} \cdot \boldsymbol{u}_{\zeta \eta}, q\right\rangle+\left\langle\kappa_{\mathrm{abs}} \boldsymbol{\nabla}_{\chi_{\zeta \eta}}, \boldsymbol{\nabla} q\right\rangle\right] \mathrm{d} t=\int_{0}^{T}\left\langle h_{\mathrm{ext}}, q\right\rangle \mathrm{d} t,
\end{array}
$$

for all $(\boldsymbol{v}, q) \in L^{2}(0, T ; \boldsymbol{V}) \times L^{2}(0, T ; Q)$, where $\hat{b}_{\eta}$ is a strictly increasing regularization of $\hat{b}$ (see $(\mathrm{A} 1)_{\eta}$ below for further properties). The next two steps prove that the regularized problem has a weak solution in an analogous sense to Definition 3.1. 
Step 2: discretization in space and time. To obtain a fully discrete counterpart of (3.4), we employ the implicit Euler scheme for the discretization in time, a conforming Galerkin finite element discretization of the elasticity equation, and a finite volume discretization of the fluid flow equation, based on two-point flux approximations $[19,20]$. We stress that such a combination of discretization methods is common in the context of poromechanics $[13,45]$, in particular in the engineering community as well as the industry.

Let $N \in \mathbb{N}$ and $\left\{t_{n} ; n=0, \ldots, N\right\}$ be an equidistant partition of the interval $[0, T]$ with constant time steps $\tau=T / N$. Furthermore, given an admissible mesh $\mathcal{T}=\{K\}_{K}, c f$., Definition 5.1, let $\boldsymbol{V}_{h} \subset \boldsymbol{V}$ denote a conforming, discrete function space for displacements. Let $Q_{h} \not \subset Q$ denote the discrete function space of piecewise constant functions. Then the discretization for time step $n \geq 1$ reads:

Problem $P_{h n}$ : given the solution at the previous time step $\left(\boldsymbol{u}_{h}^{n-1}, \chi_{h}^{n-1}\right) \in \boldsymbol{V}_{h} \times Q_{h}$, find $\left(\boldsymbol{u}_{h}^{n}, \chi_{h}^{n}\right) \in \boldsymbol{V}_{h} \times Q_{h}$ satisfying for all $\left(\boldsymbol{v}_{h}, q_{h}\right) \in \boldsymbol{V}_{h} \times Q_{h}$

$$
\begin{array}{r}
\zeta \tau^{-1} a\left(\boldsymbol{u}_{h}^{n}-\boldsymbol{u}_{h}^{n-1}, \boldsymbol{v}_{h}\right)+a\left(\boldsymbol{u}_{h}^{n}, \boldsymbol{v}_{h}\right)-\alpha\left\langle\hat{p}_{\text {pore }}\left(\chi_{h}^{n}\right), \boldsymbol{\nabla} \cdot \boldsymbol{v}_{h}\right\rangle=\left\langle\boldsymbol{f}_{\mathrm{ext}}^{n}, \boldsymbol{v}_{h}\right\rangle, \\
\left\langle\hat{b}_{\eta}\left(\chi_{h}^{n}\right)-\hat{b}_{\eta}\left(\chi_{h}^{n-1}\right), q_{h}\right\rangle+\alpha\left\langle\hat{s}_{\mathrm{w}}\left(\chi_{h}^{n}\right) \boldsymbol{\nabla} \cdot\left(\boldsymbol{u}_{h}^{n}-\boldsymbol{u}_{h}^{n-1}\right), q_{h}\right\rangle+\tau\left\langle\nabla_{h} \chi_{h}^{n}, \nabla_{h} q_{h}\right\rangle_{\kappa_{\mathrm{abs}}}=\tau\left\langle h_{\mathrm{ext}}^{n}, q_{h}\right\rangle .
\end{array}
$$

This specific choice for the discretization will turn out to be crucial due to two reasons: (i) the piecewise constant approximation of the pressure allows for choosing non-linear test functions and thereby cancelling the coupling terms in the analysis; (ii) the two-point flux approximation encoded by the discrete gradients $\boldsymbol{\nabla}_{h}$ retain the local character of the differential operator $\boldsymbol{\nabla}$. These together allow for simultaneously cancelling the coupling terms and utilizing the coercivity of the diffusion term, which will be crucial for proving existence of discrete solution via a corollary of Brouwer's fixed point theorem, and for deriving stability estimates.

Step 3: existence of a weak solution to the regularized model. Based on the discrete values $\left\{\left(\boldsymbol{u}_{h}^{n}, \chi_{h}^{n}\right)\right\}_{n}$, we define suitable interpolations in time, $\left(\boldsymbol{u}_{h \tau}, \chi_{h \tau}\right)$, yielding approximations of $\left(\boldsymbol{u}_{\zeta \eta}, \chi_{\zeta \eta}\right)$. We remark that various interpolations are in fact introduced in the course of steps 3 and 4 . To avoid an excess in notations and for the ease of the presentation, we use the same notation, $\left(\boldsymbol{u}_{h \tau}, \chi_{h \tau}\right)$, for all interpolations throughout this section.

The goal is to show convergence (in a certain sense) of $\left\{\left(\boldsymbol{u}_{h \tau}, \chi_{h \tau}\right)\right\}_{h, \tau}$ along a monotonically decreasing sequence of pairs $(h, \tau) \rightarrow(0,0)$ (from now on denoted $h, \tau \rightarrow 0)$ towards a solution of (3.4). This is achieved using compactness arguments; however, due to the coupled and non-linear nature of (3.4), several terms require careful discussion:

- Products of independent variables as $\hat{s}_{\mathrm{w}}\left(\chi_{\zeta \eta}\right) \partial_{t} \boldsymbol{\nabla} \cdot \boldsymbol{u}_{\zeta \eta}$ and non-linearities as $\hat{p}_{\text {pore }}\left(\chi_{\zeta \eta}\right)$ require partially strong convergence.

- Since $\hat{b}_{\eta}$ is not necessarily Lipschitz continuous, it is not sufficient to show uniform stability for $\left\{\partial_{t} \chi_{h \tau}\right\}_{h, \tau}$ to conclude weak convergence of $\left\{\partial_{t} \hat{b}_{\eta}\left(\chi_{h \tau}\right)\right\}_{h, \tau}$ towards $\partial_{t} \hat{b}_{\eta}\left(\chi_{\zeta \eta}\right)$. Instead, we apply techniques from [1] utilizing an energy $\hat{B}_{\eta}$ based on $\hat{b}_{\eta}$ analogously to (4.2).

- Weak convergence (up to a subsequence) of the discrete gradients $\nabla_{h} \chi_{h \tau}$ towards $\nabla \chi_{\zeta \eta}$ is not an obvious consequence of uniform stability. For this, we apply techniques from the finite volume literature $[19,34]$.

Motivated by that, we first derive stability estimates that are uniform wrt. the discretization parameters

$$
\begin{aligned}
\left\|\boldsymbol{u}_{h \tau}\right\|_{H^{1}(0, T ; \boldsymbol{V})} & +\operatorname{esssup}_{t \in(0, T)}\left\|\chi_{h \tau}(t)\right\|_{1, \mathcal{T}}+\left\|\partial_{t} \chi_{h \tau}\right\|_{L^{2}\left(Q_{T}\right)}+\left\|\hat{p}_{\text {pore }}\left(\chi_{h \tau}\right)\right\|_{L^{2}\left(Q_{T}\right)} \\
& +\left\|\hat{B}_{\eta}\left(\chi_{h \tau}\right)\right\|_{L^{\infty}\left(0, T ; L^{1}(\Omega)\right)}+\left\|\partial_{t} \hat{b}_{\eta}\left(\chi_{h \tau}\right)\right\|_{L^{2}\left(0, T ; H^{-1}(\Omega)\right)} \leq C_{\zeta \eta}
\end{aligned}
$$

for some constant $C_{\zeta \eta}>0$ independent of $h, \tau$ - as already indicated in step 2, the specific spatial discretization is beneficial for obtaining this result. Therefore, one obtains weak convergence for subsequences (denoted the same as before) for $h, \tau \rightarrow 0$

$$
\boldsymbol{u}_{h \tau} \rightarrow \boldsymbol{u}_{\zeta \eta} \quad \text { weakly in } H^{1}(0, T ; \boldsymbol{V}),
$$




$$
\begin{aligned}
\hat{p}_{\text {pore }}\left(\chi_{h \tau}\right) & \rightarrow \hat{p}_{\text {pore }}\left(\chi_{\zeta \eta}\right) & & \text { weakly in } L^{2}\left(Q_{T}\right), \\
\partial_{t} \hat{b}_{\eta}\left(\chi_{h \tau}\right) & \rightarrow \partial_{t} \hat{b}_{\eta}\left(\chi_{\zeta \eta}\right) & & \text { weakly in } L^{2}\left(0, T ; Q^{\star}\right), \\
\hat{s}_{\mathrm{w}}\left(\chi_{h \tau}\right) \partial_{t} \boldsymbol{\nabla} \cdot \boldsymbol{u}_{h \tau} & \rightarrow \hat{s}_{\mathrm{w}}\left(\chi_{\zeta \eta}\right) \partial_{t} \boldsymbol{\nabla} \cdot \boldsymbol{u}_{\zeta \eta} & & \text { weakly in } L^{2}\left(Q_{T}\right) .
\end{aligned}
$$

Moreover, by employing finite volume techniques the following convergence of the discrete diffusion term can be showed

$$
\int_{0}^{T}\left\langle\nabla_{h} \chi_{h \tau}, \nabla_{h} q_{h}\right\rangle_{\kappa_{\mathrm{abs}}} \mathrm{d} t \rightarrow \int_{0}^{T}\left\langle\nabla \chi_{\zeta \eta}, \nabla q\right\rangle_{\kappa_{\mathrm{abs}}} \mathrm{d} t
$$

for arbitrary discrete test functions $q_{h}$, which strongly converge towards continuous functions $q$. Here, $\langle\cdot, \cdot\rangle_{\kappa_{\mathrm{abs}}}$ denotes suitably defined $\kappa_{\text {abs }}$-weighted scalar products. Finally, the limit, $\left(\boldsymbol{u}_{\zeta \eta}, \chi_{\zeta \eta}\right)$, can be identified as weak solution of the regularized problem (3.4).

Step 4: increased regularity for the weak solution of the regularized model. When discussing the limit $\zeta \rightarrow 0$ in step 5 , it will be beneficial to have access to the derivative in time of the mechanics equation (3.4a). Under the additional non-degeneracy condition (ND2), stating that $\hat{p}_{\text {pore }}$ is Lipschitz continuous, an increased regularity can be showed for the weak solution of the regularized model, $\left(\boldsymbol{u}_{\zeta \eta}, \chi_{\zeta \eta}\right)$. For instance, for all $\boldsymbol{v} \in$ $L^{2}(0, T ; \boldsymbol{V})$ it holds that

$$
\int_{0}^{T}\left[\zeta a\left(\partial_{t t} \boldsymbol{u}_{\zeta \eta}, \boldsymbol{v}\right)+a\left(\partial_{t} \boldsymbol{u}_{\zeta \eta}, \boldsymbol{v}\right)-\alpha\left\langle\partial_{t} \hat{p}_{\text {pore }}\left(\chi_{\zeta \eta}\right), \boldsymbol{\nabla} \cdot \boldsymbol{v}\right\rangle\right] \mathrm{d} t=\int_{0}^{T}\left\langle\partial_{t} \boldsymbol{f}_{\text {ext }}, \boldsymbol{v}\right\rangle \mathrm{d} t .
$$

The proof follows the same line of argumentation as step 3. First a fully discrete counterpart of (3.6) is constructed by considering differences of (3.5a) between subsequent time steps

$$
\begin{aligned}
& \zeta \tau^{-1} a\left(\boldsymbol{u}_{h}^{n}-2 \boldsymbol{u}_{h}^{n-1}+\boldsymbol{u}_{h}^{n-2}, \boldsymbol{v}_{h}\right)+a\left(\boldsymbol{u}_{h}^{n}-\boldsymbol{u}_{h}^{n-1}, \boldsymbol{v}_{h}\right) \\
& \quad-\alpha\left\langle\hat{p}_{\text {pore }}\left(\chi_{h}^{n}\right)-\hat{p}_{\text {pore }}\left(\chi_{h}^{n-1}\right), \boldsymbol{\nabla} \cdot \boldsymbol{v}_{h}\right\rangle=\left\langle\boldsymbol{f}_{\text {ext }}^{n}-\boldsymbol{f}_{\text {ext }}^{n-1}, \boldsymbol{v}_{h}\right\rangle \text { for } \boldsymbol{v}_{h} \in \boldsymbol{V}_{h} .
\end{aligned}
$$

In addition, suitable interpolations $\hat{\boldsymbol{u}}_{t, h \tau}$ and $\hat{p}_{\text {pore }, h \tau}$ of the discrete values $\left\{\tau^{-1}\left(\boldsymbol{u}_{h}^{n}-\boldsymbol{u}_{h}^{n-1}\right)\right\}_{n}$ and $\left\{\hat{p}_{\text {pore }}\left(\chi_{h}^{n}\right)\right\}_{n}$, respectively, define approximations of $\partial_{t} \boldsymbol{u}_{\zeta \eta}$ and $\hat{p}_{\text {pore }}\left(\chi_{\zeta \eta}\right)$. The uniform stability estimate

$$
\left\|\partial_{t} \hat{\boldsymbol{u}}_{t, h \tau}\right\|_{L^{2}(0, T ; \boldsymbol{V})}^{2}+\left\|\partial_{t} \boldsymbol{u}_{h \tau}\right\|_{L^{2}(0, T ; \boldsymbol{V})}^{2}+\left\|\partial_{t} \hat{p}_{\mathrm{pore}, h \tau}\right\|_{L^{2}\left(Q_{T}\right)}^{2} \leq C_{\zeta \eta}
$$

guarantees the weak convergences

$$
\begin{aligned}
\partial_{t} \hat{\boldsymbol{u}}_{t, h \tau} & \rightarrow \partial_{t t} \boldsymbol{u}_{\zeta \eta}, & & \text { weakly in } L^{2}(0, T ; \boldsymbol{V}), \\
\partial_{t} \boldsymbol{u}_{h \tau} & \rightarrow \partial_{t} \boldsymbol{u}_{\zeta \eta}, & & \text { weakly in } L^{2}(0, T ; \boldsymbol{V}), \\
\partial_{t} \hat{p}_{\text {pore }}(\chi)_{h \tau} & \rightarrow \partial_{t} \hat{p}_{\text {pore }}\left(\chi_{\zeta \eta}\right), & & \text { weakly in } L^{2}\left(Q_{T}\right)
\end{aligned}
$$

up to subsequences, for $h, \tau \rightarrow 0$. Finally, one can identify (3.6) in the limit.

Step 5: vanishing regularization in the mechanics equation. For each $\zeta, \eta>0$, there exists a solution $\left(\boldsymbol{u}_{\zeta \eta}, \chi_{\zeta \eta}\right)$ to (3.4). For the discussion of the limit $\zeta \rightarrow 0$, we employ compactness arguments similar to step 3. We derive the uniform stability estimates

$$
\begin{aligned}
\left\|\boldsymbol{u}_{\zeta \eta}\right\|_{H^{1}(0, T ; \boldsymbol{V})} & +\left\|\chi_{\zeta \eta}\right\|_{L^{\infty}(0, T ; Q)}+\left\|\hat{p}_{\text {pore }}\left(\chi_{\zeta \eta}\right)\right\|_{L^{2}\left(Q_{T}\right)} \\
& +\left\|\hat{B}_{\eta}\left(\chi_{\zeta \eta}\right)\right\|_{L^{\infty}\left(0, T ; L^{1}(\Omega)\right)}+\left\|\partial_{t} \hat{b}_{\eta}\left(\chi_{\zeta \eta}\right)\right\|_{L^{2}\left(0, T ; H^{-1}(\Omega)\right)} \leq C,
\end{aligned}
$$

and

$$
\left\|\partial_{t} \chi_{\zeta \eta}\right\|_{L^{2}\left(Q_{T}\right)} \leq C_{\eta}
$$


In order to derive (3.7), we take inspiration from the analysis of the linear Biot equations in [25] and utilize the differentiated-in-time momentum equation (3.6). We use $\boldsymbol{v}=\partial_{t} \boldsymbol{u}_{\zeta \eta}$ as test function in (3.6) (essentially generating $\left\|\partial_{t} \boldsymbol{u}_{\zeta \eta}\right\|_{H^{1}(0, T ; V)}$ ), and $q=\partial_{t} \chi_{\zeta \eta}$ in the fluid flow equation (3.4b), tested with (allowing for simple discussion of the transformed diffusion term). This generates mostly positive terms, which in principle would lead to (3.7). However, unlike in the linear Biot case, the coupling terms do not cancel, and leave behind a nonpositive term. The main idea to recover (3.7) is then to compensate the non-positive term, under a condition on the data and constitutive laws. For this, we apply the mean inequality $\|\boldsymbol{v}\|_{\boldsymbol{V}}^{2} \geq K_{\mathrm{dr}}\|\boldsymbol{\nabla} \cdot \boldsymbol{v}\|_{L^{2}}^{2}$, the binomial identity (App. B.2), together with the non-degeneracy condition (ND3) to obtain

$$
\underbrace{\left\|\partial_{t} \boldsymbol{u}_{\zeta \eta}\right\|_{L^{2}(0, T ; \boldsymbol{V})}^{2}}_{\geq \frac{1}{2}\left\|\partial_{t} \boldsymbol{u}_{\zeta \eta}\right\|_{L^{2}(0, T ; \boldsymbol{V})}^{2}+\frac{1}{2} K_{\mathrm{dr}}\left\|\partial_{t} \boldsymbol{\nabla} \cdot \boldsymbol{u}_{\zeta \eta}\right\|_{L^{2}\left(0, T ; L^{2}(\Omega)\right)}^{2}}+\int_{0}^{T}\left\langle\partial_{t} \hat{b}_{\eta}\left(\chi_{\zeta \eta}\right), \partial_{t} \chi_{\zeta \eta}\right\rangle+\alpha \int_{0}^{T}\left\langle\hat{s}_{\mathrm{w}} \partial_{t} \chi_{\zeta \eta}-\partial_{t} \hat{p}_{\text {pore }}, \partial_{t} \boldsymbol{\nabla} \cdot \boldsymbol{u}_{\zeta \eta}\right\rangle \geq \frac{1}{2}\left\|\partial_{t} \boldsymbol{u}_{\zeta \eta}\right\|_{L^{2}(0, T ; \boldsymbol{V})}^{2} .
$$

This intermediate calculation allows to drop the coupling terms in the analysis and retrieve the uniform bound (3.7) at the cost of (ND3). This calculation carefully demonstrates the interpretation of uniform compressibility provided by (ND3), $c f$., the physical interpretation discussed in Section 3.2.

With this, letting $\zeta \rightarrow 0$, one obtains for subsequences (denoted the same as before)

$$
\begin{aligned}
\boldsymbol{u}_{\zeta \eta} & \rightarrow \boldsymbol{u}_{\eta} & & \text { weakly in } L^{2}(0, T ; \boldsymbol{V}), \\
\partial_{t} \boldsymbol{u}_{\zeta \eta} & \rightarrow \partial_{t} \boldsymbol{u}_{\eta} & & \text { weakly in } L^{2}(0, T ; \boldsymbol{V}), \\
\zeta \partial_{t} \boldsymbol{u}_{\zeta \eta} & \rightarrow \mathbf{0} & & \text { weakly in } L^{2}(0, T ; \boldsymbol{V}), \\
\chi_{\zeta \eta} & \rightarrow \chi_{\eta} & & \text { weakly in } L^{\infty}(0, T ; Q), \\
\hat{p}_{\text {pore }}\left(\chi_{\zeta \eta}\right) & \rightarrow \hat{p}_{\text {pore }}\left(\chi_{\eta}\right) & & \text { weakly in } L^{2}\left(Q_{T}\right), \\
\hat{s}_{\mathrm{W}}\left(\chi_{\zeta \eta}\right) \partial_{t} \boldsymbol{\nabla} \cdot \boldsymbol{u}_{\zeta \eta} & \rightarrow \hat{s}_{\mathrm{w}}\left(\chi_{\eta}\right) \partial_{t} \boldsymbol{\nabla} \cdot \boldsymbol{u}_{\eta} & & \text { weakly in } L^{2}\left(Q_{T}\right), \\
\partial_{t} \hat{b}_{\eta}\left(\chi_{\zeta \eta}\right) & \rightarrow \partial_{t} \hat{b}_{\eta}\left(\chi_{\eta}\right) & & \text { weakly in } L^{2}\left(0, T ; Q^{\star}\right) .
\end{aligned}
$$

Finally, it is straightforward to see that the limit $\left(\boldsymbol{u}_{\eta}, \chi_{\eta}\right)$ is a weak solution of (3.4) for $\zeta=0$.

We underline, that for showing (3.9), the time-continuous character of the variational problem is required. It is not obvious how to use a similar strategy on time-discrete level. Therefore, step 5 has been performed separately from steps 3 and 4 .

Step 6: vanishing regularization in the flow equation. In the presence of fluid or solid grain compressibility in the original formulation, i.e., $c_{\mathrm{w}}>0$ or $\frac{1}{N}>0$, respectively, this final step is obsolete. Otherwise, we consider the limit process $\eta \rightarrow 0$ for the sequence of solutions $\left\{\left(\boldsymbol{u}_{\eta}, \chi_{\eta}\right)\right\}_{\eta}$, derived in step 5 . The overall idea is the same as in step 5 , namely to obtain estimates that are uniform wrt. $\eta$ and to use compactness arguments. Referring to (3.7), the following estimate is uniform in $\eta$

$$
\begin{aligned}
\left\|\boldsymbol{u}_{\eta}\right\|_{H^{1}(0, T ; \boldsymbol{V})} & +\left\|\chi_{\eta}\right\|_{L^{\infty}\left(0, T ; H_{0}^{1}(\Omega)\right)}+\left\|\hat{p}_{\text {pore }}\left(\chi_{\eta}\right)\right\|_{L^{2}\left(Q_{T}\right)} \\
& +\left\|\hat{B}_{\eta}\left(\chi_{\eta}\right)\right\|_{L^{\infty}\left(0, T ; L^{1}(\Omega)\right)}+\left\|\partial_{t} \hat{b}_{\eta}\left(\chi_{\eta}\right)\right\|_{L^{2}\left(0, T ; H^{-1}(\Omega)\right)} \leq C .
\end{aligned}
$$

For estimating $\partial_{t} \chi_{\eta}$, we first show that the time derivative of the mechanics equation (3.5a) is well-defined for $\zeta=0$, i.e., it holds for all $\boldsymbol{v} \in L^{2}(0, T ; \boldsymbol{V})$ that

$$
\int_{0}^{T} a\left(\partial_{t} \boldsymbol{u}_{\eta}, \boldsymbol{v}\right) \mathrm{d} t-\int_{0}^{T} \alpha\left\langle\partial_{t} \hat{p}_{\text {pore }}\left(\chi_{\eta}\right), \boldsymbol{\nabla} \cdot \boldsymbol{v}\right\rangle \mathrm{d} t=\int_{0}^{T}\left\langle\partial_{t} \boldsymbol{f}_{\text {ext }}, \boldsymbol{v}\right\rangle \mathrm{d} t .
$$

Since $\left\|\partial_{t} \chi_{\eta}\right\| \lesssim\left\|\partial_{t} \hat{p}_{\text {pore }}\left(\chi_{\eta}\right)\right\|$, the uniform stability for $\partial_{t} \chi_{\eta}$ follows by an inf-sup argument (3.11), and the stability bound (3.10). Due to the lack of a suitable bound on $\partial_{t t} \boldsymbol{u}_{\zeta \eta}$ in step 5 , this approach only works for 
$\zeta=0$. Standard compactness arguments allow for extracting subsequences (again denoted as before) such that for $\eta \rightarrow 0$ it holds that

$$
\begin{aligned}
\boldsymbol{u}_{\eta} & \rightarrow \boldsymbol{u} & & \text { weakly in } L^{2}(0, T ; \boldsymbol{V}), \\
\chi_{\eta} & \rightarrow \chi & & \text { weakly in } L^{\infty}(0, T ; Q), \\
\hat{p}_{\text {pore }}\left(\chi_{\eta}\right) & \rightarrow \hat{p}_{\text {pore }}(\chi) & & \text { weakly in } L^{2}\left(Q_{T}\right), \\
\hat{s}_{\mathrm{w}}\left(\chi_{\eta}\right) \partial_{t} \boldsymbol{\nabla} \cdot \boldsymbol{u}_{\eta} & \rightarrow \hat{s}_{\mathrm{w}}(\chi) \partial_{t} \boldsymbol{\nabla} \cdot \boldsymbol{u} & & \text { weakly in } L^{2}\left(Q_{T}\right), \\
\partial_{t} \hat{b}_{\eta}\left(\chi_{\eta}\right) & \rightarrow \partial_{t} \hat{b}(\chi) & & \text { weakly in } L^{2}\left(0, T ; Q^{\star}\right) .
\end{aligned}
$$

Ultimately, $(\boldsymbol{u}, \chi)$ can be identified as a weak solution to the unsaturated poroelasticity model in the sense of Definition 3.1. This finishes the proof of Theorem 3.4.

\section{Step 1: Physical Regularization - Secondary Consolidation And Enhanced GRAIN COMPRESSIBILITY}

We introduce a physical regularization of the weak formulation (3.2) by enhancing the mechanics and the flow equations. Specifically, we let $\zeta>0$ and $\eta>0$ be two regularization parameters. We include secondary consolidation, which effectively adds a linear viscoelastic contribution in the mechanics equation of the form $\zeta a\left(\partial_{t} \boldsymbol{u}, \boldsymbol{v}\right)$. Additionally, we assume non-vanishing solid grain compressibility by defining the regularization $\hat{b}_{\eta}$ of $\hat{b}$ as

$$
\hat{b}_{\eta}(\chi):=\hat{b}(\chi)+\eta \int_{0}^{\hat{p}_{\mathrm{w}}(\chi)} s_{\mathrm{w}}(p) p_{\text {pore }}^{\prime}(p) \mathrm{d} p
$$

i.e., $\hat{b}_{\eta}$ has the same structure as $\hat{b}$, but with $\frac{1}{N}+\eta$ replacing $\frac{1}{N}$ refering to the physical example (2.6).

Refering to Section 3.2, the function $\hat{b}_{\eta}$ still satisfies (A1). Additionally, now the following growth condition holds

$(\mathrm{A} 1)_{\eta}$ There exists a $\hat{b}_{\mathrm{m}}>0$ s.t. $\hat{b}_{\mathrm{m}}\left\|\chi_{1}-\chi_{2}\right\|_{L^{2}(\Omega)}^{2} \leq\left\langle\hat{b}_{\eta}\left(\chi_{1}\right)-\hat{b}_{\eta}\left(\chi_{2}\right), \chi_{1}-\chi_{2}\right\rangle$ for all $\chi_{1}, \chi_{2} \in L^{2}\left(Q_{T}\right)$,

$c f$., also Appendix A. In the subsequent discussion, a growth condition for $\hat{b}_{\eta}$ (or $\hat{b}$ ) of type (A1) $\eta$ will be required in order to utilize strong compactness arguments for the pressure variable. Note, that if $\min \left\{c_{\mathrm{w}}, \frac{1}{N}\right\}>0$ in $(2.6)$, the growth condition $(\mathrm{A} 1)_{\eta}$ is fulfilled even for $\eta=0$, and the regularization of the flow equation actually is not necessary, cf., Step 6 in Section 9.

Also (A8) can be adapted for the regularization $\hat{b}_{\eta}$. With $\bar{b}_{\eta}:=\hat{b}_{\eta} \circ\left(\frac{\hat{p}_{\text {pore }}}{\hat{s}_{\mathrm{w}}}\right)^{-1}$, we define energies $\hat{B}_{\eta}$ and $\bar{B}_{\eta}$, related to the Legendre transformations of convex potentials of $\hat{b}_{\eta}$ and $\bar{b}_{\eta}$, respectively, $c f$. , [1] or Lemma B.12. Let

$$
\begin{aligned}
& \hat{B}_{\eta}(z):=\int_{0}^{z}\left(\hat{b}_{\eta}(z)-\hat{b}_{\eta}(s)\right) \mathrm{d} s \geq 0, \\
& \bar{B}_{\eta}(z):=\int_{0}^{z}\left(\bar{b}_{\eta}(z)-\bar{b}_{\eta}(s)\right) \mathrm{d} s \geq 0 .
\end{aligned}
$$

$(\mathrm{A} 8)_{\eta}$ There exists a $\eta_{0}>0$ and $C_{0}>0$, not depending on $\eta_{0}$, such that

$$
\left.\left\|\boldsymbol{u}_{0}\right\|_{\boldsymbol{V}}^{2}+\left\|\boldsymbol{\nabla} \chi_{0}\right\|_{L^{2}(\Omega)}^{2}+\| \hat{B}_{\eta}\left(\chi_{0}\right)\right)\left\|_{L^{1}(\Omega)}+\right\| \bar{B}_{\eta}\left(\frac{\hat{p}_{\text {pore }}\left(\chi_{0}\right)}{\hat{s}_{\mathrm{w}}\left(\chi_{0}\right)}\right) \|_{L^{1}(\Omega)} \leq C_{0}
$$

for all $\eta \in\left(0, \eta_{0}\right)$. Without loss of generality, we assume $C_{0}$ in $(\mathrm{A} 8)$ and $(\mathrm{A} 8)_{\eta}$ to be the same. 
For a non-degenerate initial condition $\chi_{0}$, the additional terms in $\hat{B}_{\eta}$ and $\bar{B}_{\eta}$ can be essentially bounded by $\eta\left\|\chi_{0}\right\|_{L^{2}(\Omega)}^{2}$, which itself is bounded by (A8).

Finally, we introduce the doubly regularized unsaturated poroelasticity model by defining the notion of a weak solution.

Definition 4.1 (Weak solution of the doubly regularized model). For $\zeta>0$ and $\eta>0$, we call $\left(\boldsymbol{u}_{\zeta \eta}, \chi_{\zeta \eta}\right) \in$ $L^{2}(0, T ; \boldsymbol{V}) \times L^{2}(0, T ; Q)$ a weak solution of the doubly regularized unsaturated poroelasticity model if it satisfies:

$(\mathrm{W} 1)_{\zeta \eta} \hat{p}_{\text {pore }}\left(\chi_{\zeta \eta}\right) \in L^{2}\left(Q_{T}\right), \hat{s}_{\mathrm{w}}\left(\chi_{\zeta \eta}\right) \in L^{\infty}\left(Q_{T}\right)$.

$(\mathrm{W} 2)_{\zeta \eta} \hat{b}_{\eta}\left(\chi_{\zeta \eta}\right) \in L^{\infty}\left(0, T ; L^{1}(\Omega)\right)$ and $\partial_{t} \hat{b}_{\eta}\left(\chi_{\zeta \eta}\right) \in L^{2}\left(0, T ; Q^{\star}\right)$ such that

$$
\int_{0}^{T}\left\langle\partial_{t} \hat{b}_{\eta}\left(\chi_{\zeta \eta}\right), q\right\rangle \mathrm{d} t+\int_{0}^{T}\left\langle\hat{b}_{\eta}\left(\chi_{\zeta \eta}\right)-\hat{b}_{\eta}\left(\chi_{0}\right), \partial_{t} q\right\rangle \mathrm{d} t=0
$$

for all $q \in L^{2}(0, T ; Q) \cap W^{1,1}\left(0, T ; L^{\infty}(\Omega)\right)$ with $q(T)=0$.

(W3) $)_{\zeta \eta} \partial_{t} \boldsymbol{u}_{\zeta \eta} \in L^{2}(0, T ; \boldsymbol{V})$ such that

$$
\int_{0}^{T} a\left(\partial_{t} \boldsymbol{u}_{\zeta \eta}, \boldsymbol{v}\right) \mathrm{d} t+\int_{0}^{T} a\left(\boldsymbol{u}_{\zeta \eta}-\boldsymbol{u}_{0}, \partial_{t} \boldsymbol{v}\right) \mathrm{d} t=0
$$

for all $\boldsymbol{v} \in H^{1}(0, T ; \boldsymbol{V})$ with $\boldsymbol{v}(T)=\mathbf{0}$, where $\boldsymbol{u}_{0}$ satisfies (3.1).

$(\mathrm{W} 4)_{\zeta \eta}\left(\boldsymbol{u}_{\zeta \eta}, \chi_{\zeta \eta}\right)$ satisfies for all $(\boldsymbol{v}, q) \in L^{2}(0, T ; \boldsymbol{V}) \times L^{2}(0, T ; Q)$

$$
\begin{array}{r}
\int_{0}^{T}\left[\zeta a\left(\partial_{t} \boldsymbol{u}_{\zeta \eta}, \boldsymbol{v}\right)+a\left(\boldsymbol{u}_{\zeta \eta}, \boldsymbol{v}\right)-\alpha\left\langle\hat{p}_{\text {pore }}\left(\chi_{\zeta \eta}\right), \boldsymbol{\nabla} \cdot \boldsymbol{v}\right\rangle\right] \mathrm{d} t=\int_{0}^{T}\left\langle\boldsymbol{f}_{\text {ext }}, \boldsymbol{v}\right\rangle \mathrm{d} t \\
\int_{0}^{T}\left[\left\langle\partial_{t} \hat{b}_{\eta}\left(\chi_{\zeta \eta}\right)+\alpha \hat{s}_{\mathrm{w}}\left(\chi_{\zeta \eta}\right) \partial_{t} \boldsymbol{\nabla} \cdot \boldsymbol{u}_{\zeta \eta}, q\right\rangle+\left\langle\kappa_{\mathrm{abs}} \boldsymbol{\nabla}_{\chi_{\zeta \eta}}, \boldsymbol{\nabla} q\right\rangle\right] \mathrm{d} t=\int_{0}^{T}\left\langle h_{\mathrm{ext}}, q\right\rangle \mathrm{d} t .
\end{array}
$$

Furthermore, we call $\left(\boldsymbol{u}_{\zeta \eta}, \chi_{\zeta \eta}\right)$ a weak solution for the doubly regularized unsaturated poroelasticity model with increased regularity if it additionally satisfies $(\mathrm{W} 1)_{\zeta \eta}-(\mathrm{W} 4)_{\zeta \eta}$ and:

$(\mathrm{W} 5)_{\zeta \eta} \boldsymbol{u}_{\zeta \eta} \in H^{2}(0, T ; \boldsymbol{V})$ and $\partial_{t} \hat{p}_{\text {pore }}\left(\chi_{\zeta \eta}\right) \in L^{2}\left(Q_{T}\right)$.

(W6) $)_{\zeta \eta}$ Given that $\boldsymbol{f}_{\text {ext }} \in H^{1}\left(0, T ; \boldsymbol{V}^{\star}\right)$, for all $\boldsymbol{v} \in L^{2}(0, T ; \boldsymbol{V})$ it holds that

$$
\int_{0}^{T}\left[\zeta a\left(\partial_{t t} \boldsymbol{u}_{\zeta \eta}, \boldsymbol{v}\right)+a\left(\partial_{t} \boldsymbol{u}_{\zeta \eta}, \boldsymbol{v}\right)-\alpha\left\langle\partial_{t} \hat{p}_{\text {pore }}\left(\chi_{\zeta \eta}\right), \boldsymbol{\nabla} \cdot \boldsymbol{v}\right\rangle\right] \mathrm{d} t=\int_{0}^{T}\left\langle\partial_{t} \boldsymbol{f}_{\text {ext }}, \boldsymbol{v}\right\rangle \mathrm{d} t .
$$

We will later separately consider $\zeta \rightarrow 0$ and $\eta \rightarrow 0$. Therefore, we give the definition of a weak solution for the simply regularized unsaturated poroelasticity model, obtained for $\eta>0$ and $\zeta=0$.

Definition 4.2 (Weak solution of the simply regularized model). For $\eta>0$, we call $\left(\boldsymbol{u}_{\eta}, \chi_{\eta}\right)$ a weak solution of the simply regularized unsaturated poroelasticity model if it satisfies $(\mathrm{W} 1)_{\zeta \eta}-(\mathrm{W} 4)_{\zeta \eta}$ for $\zeta=0$.

To distinguish between the equations satisfied by the weak solution of a doubly regularized model and the one of the simply regularized one, where $\zeta=0$, we use the notation $(\mathrm{W} 1)_{\eta}-(\mathrm{W} 4)_{\eta}$.

Lemma 4.3 (Existence of a weak solution to the doubly regularized model). Let $\zeta>0$ and $\eta>0$ be given. Under the assumptions (A0)-(A8) and (ND1) there exists a weak solution to the doubly regularized unsaturated poroelasticity model, in the sense of Definition 4.1.

Proof. The assertion follows from steps 2 and 3. 
Remark 4.4. The proof of Lemma 4.3 presented in Section 6 does in fact allow for replacing the regularizing term $\zeta a\left(\partial_{t} \boldsymbol{u}, \boldsymbol{v}\right)$ in $(4.4 \mathrm{a})$ with a reduced regularization merely applied to the temporal derivative of the volumetric deformation, i.e., $\zeta\left\langle\partial_{t} \boldsymbol{\nabla} \cdot \boldsymbol{u}, \boldsymbol{\nabla} \cdot \boldsymbol{v}\right\rangle$. The statement of Lemma 4.3 remains true with an adapted version of $(\mathrm{W} 3)_{\zeta \eta}$ addressing only the initial condition for the volumetric deformation. Such regularized model is of wider interest in the literature [16,22, 28,35,42], in particular in the context of biomedical applications [5].

Lemma 4.5 (Existence of a weak solution with increased regularity for the doubly regularized model). Let $\zeta>0$ and $\eta>0$ be given. Under the assumptions (A0)-(A8) and the non-degeneracy conditions (ND1)-(ND2), the doubly regularized unsaturated poroelasticity model has a weak solution with increased regularity, in the sense of Definition 4.1.

Proof. The assertion follows from steps 2 to 4 .

Lemma 4.6 (Existence of a weak solution for the simply regularized model). Let $\eta>0$ be given. Under the assumptions (A0)-(A8) and the non-degeneracy conditions (ND1)-(ND3), the simply regularized unsaturated poroelasticity model has a weak solution with increased regularity, in the sense of Definition 4.2.

Proof. The assertion follows from step 5.

\section{Step 2: Implicit EUler NON-LineAR FEM-TPFA Discretization}

The next two sections, identified with steps 2 and 3, are providing the proof of Lemma 4.3. To this aim, we employ a discretization in space and time. We apply the implicit Euler time stepping method, combined with a conforming Galerkin finite element method for the mechanics equation (4.4a) and a cell-centered finite volume method utilizing a two point flux approximation (TPFA) for the flow equation (4.4b). In this section, we establish the existence of a fully discrete solution. We start with introducing the notations used in the discretization.

\subsection{Finite volume and finite element notation}

We use standard notations in the finite volume literature, $c f .$, e.g., [19,34]. In particular, we introduce notation for elements, faces, their measures, transmissibilities etc. We assume that the domain $\Omega$ is polygonal such that it can be discretized by an admissible mesh, as introduced in [20].

Definition 5.1 (Admissible mesh $\mathcal{T}$ ). Let $\mathcal{T}$ be a regular mesh of $\Omega$ with mesh size $h$, consisting of simplices in $2 \mathrm{D}$ or $3 \mathrm{D}$, or convex quadrilaterals in $2 \mathrm{D}$ and convex hexahedrals in $3 \mathrm{D}$. Furthermore, we introduce the following terminology:

- $K \in \mathcal{T}$ denotes a single element.

- $\mathcal{N}(K):=\{L \in \mathcal{T} \mid L \neq K, \bar{L} \cap \bar{K} \neq \emptyset\}$ denotes the set of neighboring elements of $K \in \mathcal{T}$.

$-\mathcal{E}$ denotes the set of all faces, i.e., boundaries of all elements; let $\mathcal{E}_{K}$ denote the faces of a single element $K \in \mathcal{T}$; let $\mathcal{E}_{\text {ext }}$ denote the faces lying on the boundary $\partial \Omega$.

- $K \mid L \in \mathcal{E}$ denotes the face between two neighboring elements $K, L \in \mathcal{T}$.

- $\left\{x_{K}\right\}_{K \in \mathcal{T}}$ is such that for all $K \in \mathcal{T}, L \in \mathcal{N}(K)$ the connecting line between $x_{K}$ and $x_{L}$ is perpendicular to $K \mid L$.

- $d_{K, \sigma}$ denotes the distance between center of $K$ and $\sigma \in \mathcal{E}_{K}$;

$$
d_{\sigma}= \begin{cases}d_{K, \sigma}+d_{L, \sigma}, & K \in \mathcal{T}, L \in \mathcal{N}(K), \sigma=K \mid L, \\ d_{K, \sigma}, & \sigma \in \mathcal{E}_{\mathrm{ext}} \cap \mathcal{E}_{K} .\end{cases}
$$

- $\tau_{\sigma}=|\sigma| / d_{\sigma}$ denotes the transmissibility through $\sigma \in \mathcal{E}$. 


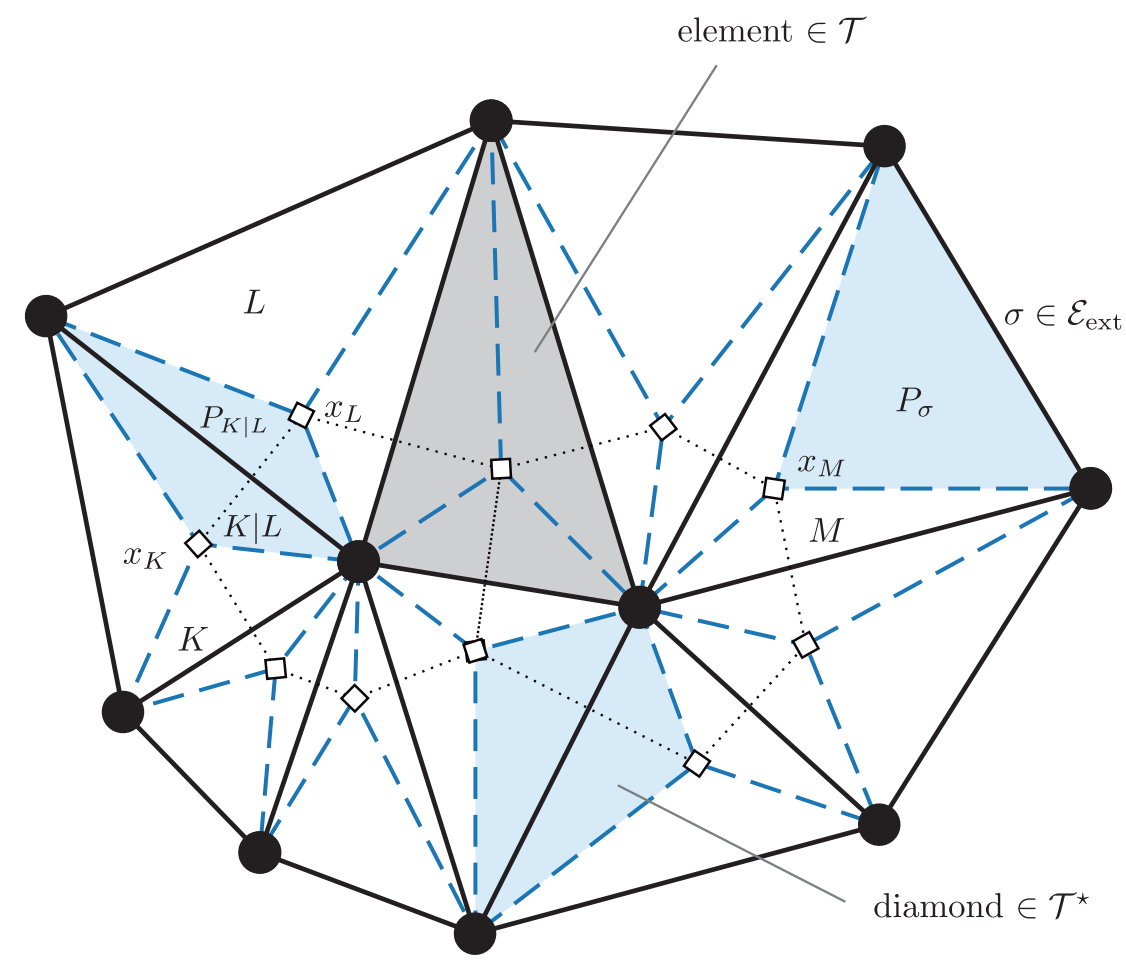

Figure 1. Admissible mesh $\mathcal{T}$ (consisting of elements) in two dimensions, together with the corresponding dual grid $\mathcal{T}^{\star}$ (consisting of diamonds).

The additional regularity property is assumed. There exists a constant $C>0$ such that

$$
\sum_{\substack{L \in \mathcal{N}(K) \\ \sigma=K \mid L}}|\sigma| d_{\sigma} \leq C|K| \quad \text { for all } K \in \mathcal{T} .
$$

In addition, we introduce a dual grid $\mathcal{T}^{\star}$ with diamonds as elements. It will be used for the approximation of heterogeneous permeability fields. Additionally, it will be utilized within the proof, to define suitable projection operators.

Definition 5.2 (Dual grid to $\mathcal{T}$ ). Let $\mathcal{T}$ be an admissible mesh, $c f$., Definition 5.1. For each face $K \mid L \in \mathcal{E}$, $K \in \mathcal{T}, L \in \mathcal{N}(K)$, define a prism $P_{K \mid L} \subset \Omega$ with $x_{K}, x_{L}$ and the vertices of $K \mid L$ as vertices. For all $\sigma \in \mathcal{E}_{\text {ext }} \cap \mathcal{E}_{K}, K \in \mathcal{T}$ define $P_{\sigma} \subset \Omega$ to be the prism with $x_{K}$ and the vertices of $\sigma$ as vertices. By construction, $\mathcal{T}^{\star}:=\left\{P_{\sigma}\right\}_{\sigma \in \mathcal{E}}$ defines a partition of $\Omega$.

Figure 1 displays a two-dimensional admissible mesh and its auxiliary dual grid.

The final discrete scheme is written in variational form. Given an admissible mesh $\mathcal{T}$, we introduce the discrete function spaces and implicitly their bases

$$
\begin{aligned}
\boldsymbol{V}_{h} & =\operatorname{span}\left\{\boldsymbol{v}_{h, i}\right\}_{i \in\left\{1, \ldots, d_{\mathrm{V}}\right\}}, \\
Q_{h} & =\operatorname{span}\left\{q_{h, j}\right\}_{j \in\left\{1, \ldots, d_{\mathrm{Q}}\right\}},
\end{aligned}
$$

providing spaces for the discrete displacement and pressure, respectively. For the analysis below, we assume that the discrete function spaces satisfy the following conditions: 
(D1) $Q_{h}$ is the space of all piecewise constant functions $\left(\mathbb{P}_{0}\right)$ on $\mathcal{T}$ and the basis $\left\{q_{h, j}\right\}_{j}$ is equal to the indicator functions of all single elements. Note $Q_{h} \not \subset Q$.

(D2) $\boldsymbol{V}_{h} \subset \boldsymbol{V}$ such that $\boldsymbol{V}_{h} \times Q_{h}$ is inf-sup stable regarding the bilinear form

$$
\boldsymbol{V}_{h} \times Q_{h} \rightarrow \mathbb{R}, \quad\left(\boldsymbol{v}_{h}, q_{h}\right) \mapsto\left\langle q_{h}, \nabla \cdot \boldsymbol{v}_{h}\right\rangle .
$$

In more detail, there exists a constant $\gamma_{\text {is }}=C_{\Omega, \text { is }}^{-1}>0$ (independent of $h$ ), such that

$$
\inf _{0 \neq q_{h} \in Q_{h}} \sup _{\boldsymbol{v}_{h} \in \boldsymbol{V}_{h}} \frac{\left\langle q_{h}, \boldsymbol{\nabla} \cdot \boldsymbol{v}_{h}\right\rangle}{\left\|q_{h}\right\|\left\|\boldsymbol{v}_{h}\right\|_{\boldsymbol{V}}} \geq \gamma_{\mathrm{is}} .
$$

In the analysis, (D1) will allow for intuitively handling non-linearities in the pressure variable. Assumption (D2) will allow for using standard inf-sup arguments. In two dimensions, e.g., piecewise quadratic elements can be used for $\boldsymbol{V}_{h}$ [6]; alternatively, both in two and three dimensions, piecewise linear elements enhanced by face bubbles result in a fairly cheap choice, in particular when utilizing localization techniques [32].

In order to define the discrete scheme, we use a discrete $H_{0}^{1}(\Omega)$ inner product and the corresponding norm, as introduced in [19], incorporating averaging of the mobility field.

Definition 5.3 (Discrete $H_{0}^{1}(\Omega)$ inner product and norm on $Q_{h}$ ). We define the inner product

$$
\left\langle\nabla_{h} \chi_{h}, \nabla_{h} q_{h}\right\rangle_{\omega}:=\sum_{K \in \mathcal{T}} \sum_{L \in \mathcal{N}(K)} \tau_{K \mid L}\{\omega\}_{K \mid L}\left(\chi_{\left.h\right|_{K}}-\chi_{\left.h\right|_{L}}\right)\left(q_{\left.h\right|_{K}}-q_{\left.h\right|_{L}}\right)+\sum_{\sigma \in \mathcal{E}_{\text {ext }} \cap \mathcal{E}_{K}} \tau_{K, \sigma}\{\omega\}_{\sigma} \chi_{\left.h\right|_{K}} q_{h_{\mid}}{ }_{K}
$$

for any $\chi_{h}, q_{h} \in Q_{h}$, where the the weight $\omega$ evaluated at faces is approximated as weighted average incorporating the neighboring elements $\{\omega\}_{\sigma}:=\frac{1}{\left|P_{\sigma}\right|} \int_{P_{\sigma}} \omega(x) \mathrm{d} x, \sigma \in \mathcal{E}$, utilizing the dual mesh $\mathcal{T}^{\star}$. In addition, we define the induced norm $\|\cdot\|_{1, \mathcal{T}, \omega}:=\left\langle\nabla_{h}, \nabla_{h} \cdot\right\rangle_{\omega}^{1 / 2}$, as well as the special case for $\omega_{1}:=\omega \equiv 1,\|\cdot\|_{1, \mathcal{T}}:=\left\langle\nabla_{h} \cdot \nabla_{h} \cdot\right\rangle_{\omega_{1}}^{1 / 2}$.

Remark 5.4 (Two-point flux approximation). Let $K \in \mathcal{T}$ be a single element. When choosing $q_{h}=\mathbf{1}_{K}$ as the indicator function of the element $K$, defined as $\mathbf{1}_{K}(x)=1$ if $x \in K$ and $\mathbf{1}_{K}(x)=0$ otherwise, simple calculations yield

$$
\left\langle\boldsymbol{\nabla}_{h} \chi_{h}, \boldsymbol{\nabla}_{h} \mathbf{1}_{K}\right\rangle_{\omega}=\sum_{L \in \mathcal{N}(K)} t_{K \mid L}\left(\chi_{\left.h\right|_{K}}-\chi_{\left.h\right|_{L}}\right)+\sum_{\sigma \in \mathcal{E}_{\text {ext }} \cap \mathcal{E}_{K}} t_{K, \sigma} \chi_{\left.h\right|_{K}}
$$

Hence, a standard two point flux approximation with averaged transmissibilities $t_{K \mid L}=\frac{2|\sigma|\{\omega\}_{\sigma}}{d_{\sigma}}$ for neighboring cells $K, L$ with common face $\sigma=K \mid L$; and $t_{K, \sigma}=\frac{|\sigma|\{\omega\}_{K, \sigma}}{d_{K, \sigma}}$ for boundary faces $\sigma$.

A discrete Poincaré inequality is proved in [19] for $\|\cdot\|_{1, \mathcal{T}}$, introducing a discrete Poincaré constant $C_{\Omega, \mathrm{DP}}>0$ such that

$$
\left\|q_{h}\right\| \leq C_{\Omega, \mathrm{DP}}\left\|q_{h}\right\|_{1, \mathcal{T}} \quad \text { for all } q_{h} \in Q_{h},
$$

cf., Lemma B.1. A similar result also holds for $\|\cdot\|_{1, \mathcal{T}, \omega}$.

\subsection{Approximation of source terms and initial conditions}

Let $0=t_{0}<t_{1}<\ldots<t_{N}=T$ define a partition of the time interval $(0, T)$ with constant time step size $\tau=t_{n}-t_{n-1}, n \in \mathbb{N}$. We interpolate the source terms at discrete time steps. Let

$$
\boldsymbol{f}_{\text {ext }}^{n}:=\frac{1}{\tau} \int_{t_{n-1}}^{t_{n}} \boldsymbol{f}_{\mathrm{ext}}(t) \mathrm{d} t
$$




$$
h_{\text {ext }}^{n}:=\frac{1}{\tau} \int_{t_{n-1}}^{t_{n}} h_{\text {ext }}(t) \mathrm{d} t .
$$

Discrete initial conditions are chosen to imitate the compatibility assumption (3.1). Let $\chi_{h}^{0} \in Q_{h}$ be defined by the piecewise constant projection of $\chi^{0}$, i.e., on $K \in \mathcal{T}$, we define

$$
\chi_{\left.h\right|_{K}}^{0}:=\frac{1}{|K|} \int_{K} \chi^{0} \mathrm{~d} x .
$$

As $\chi_{0} \in L^{2}(\Omega), c f .,(\mathrm{A} 8)_{\eta}$, it follows by classical approximation theory for $h \rightarrow 0$

$$
\chi_{h}^{0} \rightarrow \chi_{0} \text { strongly in } L^{2}(\Omega),
$$

and it holds that $\left\|\chi_{h}^{0}\right\|_{1, \mathcal{T}, \kappa_{\mathrm{abs}}} \leq C\left\|\chi_{0}\right\|_{1}$ for some constant $C>0, c f .$, e.g., [20]. Furthermore, since $\hat{p}_{\text {pore }} \in C(\mathbb{R})$, $c f .,(\mathrm{A} 3)$, and $\hat{p}_{\text {pore }}\left(\chi_{0}\right) \in L^{2}(\Omega), c f .,(\mathrm{A} 8)_{\eta}$, it follows for $h \rightarrow 0$

$$
\hat{p}_{\text {pore }}\left(\chi_{h}^{0}\right) \rightarrow \hat{p}_{\text {pore }}\left(\chi_{0}\right) \text { strongly in } L^{2}(\Omega),
$$

similarly for $\left\{\hat{B}_{\eta}\left(\chi_{h}^{0}\right)\right\}_{h}$ and $\left\{\bar{B}_{\eta}\left(\frac{\hat{p}_{\text {pore }}\left(\chi_{h}^{0}\right)}{\hat{s}_{\mathrm{w}}\left(\chi_{h}^{0}\right)}\right)\right\}_{h}$. Furthermore, we define the initial approximate displacement $\boldsymbol{u}_{h}^{0} \in \boldsymbol{V}_{h}$ to satisfy the compatibility condition

$$
a\left(\boldsymbol{u}_{h}^{0}, \boldsymbol{v}_{h}\right)-\alpha\left\langle\hat{p}_{\text {pore }}\left(\chi_{h}^{0}\right), \boldsymbol{\nabla} \cdot \boldsymbol{v}_{h}\right\rangle=\left\langle\boldsymbol{f}_{\text {ext }}(0), \boldsymbol{v}_{h}\right\rangle, \text { for all } \boldsymbol{v}_{h} \in \boldsymbol{V}_{h} .
$$

Using standard finite element techniques and the convergence of $\chi_{h}^{0}$ it follows as $h \rightarrow 0$

$$
\boldsymbol{u}_{h}^{0} \rightarrow \boldsymbol{u}_{0} \text { strongly in } \boldsymbol{V}
$$

with $\boldsymbol{u}_{0}$ defined in (W3). All in all, due to the convergence, (A8) $\eta$ also applies on discrete level.

$(\mathrm{A} 8)_{\eta, h}$ For bounded $\eta>0$, there exists a constant $C_{0}>0$ (wlog. the same as in (A8)) such that

$$
\left\|\boldsymbol{u}_{h}^{0}\right\|_{\boldsymbol{V}}^{2}+\left\|\chi_{h}^{0}\right\|_{1, \mathcal{T}, \kappa_{\mathrm{abs}}}^{2}+\left\|\hat{B}_{\eta}\left(\chi_{h}^{0}\right)\right\|_{L^{1}(\Omega)}+\left\|\bar{B}_{\eta}\left(\frac{\hat{p}_{\text {pore }}\left(\chi_{h}^{0}\right)}{\hat{s}_{\mathrm{w}}\left(\chi_{h}^{0}\right)}\right)\right\|_{L^{1}(\Omega)} \leq C_{0} .
$$

\subsection{Approximation of the evolutionary problem}

The discretization of (4.4) is defined by the Galerkin method combined with the standard implicit Euler time discretization: for $n \geq 1$, given $\left(\boldsymbol{u}_{h}^{n-1}, \chi_{h}^{n-1}\right) \in \boldsymbol{V}_{h} \times Q_{h}$, find $\left(\boldsymbol{u}_{h}^{n}, \chi_{h}^{n}\right) \in \boldsymbol{V}_{h} \times Q_{h}$ satisfying for all $\left(\boldsymbol{v}_{h}, q_{h}\right) \in \boldsymbol{V}_{h} \times Q_{h}$

$$
\begin{aligned}
& \zeta \tau^{-1} a\left(\boldsymbol{u}_{h}^{n}-\boldsymbol{u}_{h}^{n-1}, \boldsymbol{v}_{h}\right)+a\left(\boldsymbol{u}_{h}^{n}, \boldsymbol{v}_{h}\right)-\alpha\left\langle\hat{p}_{\text {pore }}\left(\chi_{h}^{n}\right), \boldsymbol{\nabla} \cdot \boldsymbol{v}_{h}\right\rangle=\left\langle\boldsymbol{f}_{\mathrm{ext}}^{n}, \boldsymbol{v}_{h}\right\rangle, \\
& \left\langle\hat{b}_{\eta}\left(\chi_{h}^{n}\right)-\hat{b}_{\eta}\left(\chi_{h}^{n-1}\right), q_{h}\right\rangle+\alpha\left\langle\hat{s}_{\mathrm{w}}\left(\chi_{h}^{n}\right) \boldsymbol{\nabla} \cdot\left(\boldsymbol{u}_{h}^{n}-\boldsymbol{u}_{h}^{n-1}\right), q_{h}\right\rangle+\tau\left\langle\nabla_{h} \chi_{h}^{n}, \nabla_{h} q_{h}\right\rangle_{\kappa_{\mathrm{abs}}}=\tau\left\langle h_{\mathrm{ext}}^{n}, q_{h}\right\rangle .
\end{aligned}
$$

Remark 5.5 (Finite volume formulation of the discrete flow problem). Based on Remark 5.4, the discrete variational formulation of the fluid flow equation $(5.5 \mathrm{~b})$ can be equivalently formulated in a standard finite volume fashion. More precisely, if $\chi_{K}:=\chi_{\left.h\right|_{K}}$ denotes the value of $\chi_{h}$ in the cell $K \in \mathcal{T}$, then (5.5b) becomes

$$
\begin{aligned}
& |K|\left(\hat{b}_{\eta}\left(\chi_{K}^{n}\right)-\hat{b}_{\eta}\left(\chi_{K}^{n-1}\right)\right)+\alpha \hat{s}_{\mathrm{w}}\left(\chi_{K}^{n}\right) \int_{K} \boldsymbol{\nabla} \cdot\left(\boldsymbol{u}_{h}^{n}-\boldsymbol{u}_{h}^{n-1}\right) \mathrm{d} x \\
& \quad+\tau \sum_{L \in \mathcal{N}(K)} t_{K \mid L}\left(\chi_{K}-\chi_{L}\right)+\tau \sum_{\sigma \in \mathcal{E}_{\text {ext }} \cap \mathcal{E}_{K}} t_{K, \sigma} \chi_{K}=\tau \int_{K} h_{\text {ext }}^{n} \mathrm{~d} x
\end{aligned}
$$

for all $K \in \mathcal{T}$. and with the transmissibilities $t_{K \mid L}$ and $t_{K, \sigma}$ as introduced in Remark 5.4. For the remaining discussion the variational formulation (5.5b) is more convenient and is therefore used. 
Lemma 5.6 (Existence of a discrete solution). Let $n \geq 1$. (A0)-(A8), (ND1), and (D1)-(D2) hold true. Then there exists a discrete solution $\left(\boldsymbol{u}_{h}^{n}, \chi_{h}^{n}\right) \in \boldsymbol{V}_{h} \times Q_{h}$ satisfying (5.5), and

$$
\left\|\bar{B}_{\eta}\left(\frac{\hat{p}_{\text {pore }}\left(\chi_{h}^{n}\right)}{\hat{s}_{\mathrm{w}}\left(\chi_{h}^{n}\right)}\right)\right\|_{L^{1}(\Omega)}+\left\|\boldsymbol{u}_{h}^{n}\right\|_{\boldsymbol{V}}^{2}<\infty \text { for all } n \geq 1 .
$$

Proof. The proof is by induction. We present only the general step, since the proof for $n=1$ is similar. We employ a corollary of Brouwer's fixed point theorem, cf., Lemma B.4, to show the existence of a solution of a non-linear algebraic system, which is equivalent to (5.5).

Introduction of a pressure-reduced algebraic problem. We introduce an isomorphism between the discrete function space corresponding to the fluid pressure $\chi$ and a suitable coefficient vector space

$$
\chi_{h}: \mathbb{R}^{d_{\mathrm{Q}}} \rightarrow Q_{h}, \quad \boldsymbol{\beta} \mapsto \sum_{j=1}^{d_{Q}}\left(\frac{\hat{p}_{\text {pore }}}{\hat{s}_{\mathrm{w}}}\right)^{-1}\left(\beta_{j}\right) q_{h, j} .
$$

Due to (A4), $\chi_{h}$ is well-defined. Similarly, let

$$
\boldsymbol{u}_{h}: \mathbb{R}^{d_{\mathrm{V}}} \rightarrow \boldsymbol{V}_{h}, \quad \boldsymbol{\alpha} \mapsto \sum_{i=1}^{d_{V}} \alpha_{i} \boldsymbol{v}_{h, i}
$$

For given $\boldsymbol{\beta} \in \mathbb{R}^{d_{\mathrm{Q}}}$, define $\boldsymbol{\alpha}=\boldsymbol{\alpha}(\boldsymbol{\beta}) \in \mathbb{R}^{d_{\mathrm{V}}}$ to be the unique solution to

$$
\zeta \tau^{-1} a\left(\boldsymbol{u}_{h}(\boldsymbol{\alpha})-\boldsymbol{u}_{h}^{n-1}, \boldsymbol{v}_{h}\right)+a\left(\boldsymbol{u}_{h}(\boldsymbol{\alpha}), \boldsymbol{v}_{h}\right)=\left\langle\boldsymbol{f}^{n}, \boldsymbol{v}_{h}\right\rangle+\alpha\left\langle\hat{p}_{\text {pore }}\left(\chi_{h}(\boldsymbol{\beta})\right), \boldsymbol{\nabla} \cdot \boldsymbol{v}_{h}\right\rangle \text {, for all } \boldsymbol{v}_{h} \in \boldsymbol{V}_{h} .
$$

Finally, we define $\boldsymbol{F}: \mathbb{R}^{d_{Q}} \rightarrow \mathbb{R}^{d_{Q}}$ component-wise by

$$
\begin{aligned}
F_{j}(\boldsymbol{\beta})= & \left\langle\hat{b}_{\eta}\left(\chi_{h}(\boldsymbol{\beta})\right)-\hat{b}_{\eta}\left(\chi_{h}^{n-1}\right), q_{h, j}\right\rangle+\alpha\left\langle\hat{s}_{\mathrm{w}}\left(\chi_{h}(\boldsymbol{\beta})\right) \boldsymbol{\nabla} \cdot\left(\boldsymbol{u}_{h}(\boldsymbol{\alpha}(\boldsymbol{\beta}))-\boldsymbol{u}_{h}^{n-1}\right), q_{h, j}\right\rangle \\
& +\tau\left\langle\boldsymbol{\nabla}_{h} \chi_{h}(\boldsymbol{\beta}), \boldsymbol{\nabla}_{h} q_{h, j}\right\rangle_{\kappa_{\mathrm{abs}}}-\tau\left\langle h_{\mathrm{ext}}^{n}, q_{h, j}\right\rangle, \quad j \in\left\{1, \ldots, d_{\mathrm{Q}}\right\} .
\end{aligned}
$$

We note, the existence of a discrete solution of equation (5.5) is equivalent to the existence of $\boldsymbol{\beta} \in \mathbb{R}^{d_{\mathrm{Q}}}$, satisfying $\boldsymbol{F}(\boldsymbol{\beta})=\mathbf{0}$. To prove the existence of a zero of $\boldsymbol{F}$, we employ Lemma B.4; we consider the expression

$$
\begin{aligned}
\langle\boldsymbol{F}(\boldsymbol{\beta}), \boldsymbol{\beta}\rangle= & \left\langle\hat{b}_{\eta}\left(\chi_{h}(\boldsymbol{\beta})\right)-\hat{b}_{\eta}\left(\chi_{h}^{n-1}\right), \frac{\hat{p}_{\text {pore }}\left(\chi_{h}(\boldsymbol{\beta})\right)}{\hat{s}_{\mathrm{w}}\left(\chi_{h}(\boldsymbol{\beta})\right)}\right\rangle+\alpha\left\langle\boldsymbol{\nabla} \cdot\left(\boldsymbol{u}_{h}(\boldsymbol{\alpha})-\boldsymbol{u}_{h}^{n-1}\right), \hat{p}_{\text {pore }}\left(\chi_{h}(\boldsymbol{\beta})\right)\right\rangle \\
& +\tau\left\langle\boldsymbol{\nabla}_{h} \chi(\boldsymbol{\beta}), \boldsymbol{\nabla}_{h} \frac{\hat{p}_{\text {pore }}\left(\chi_{h}(\boldsymbol{\beta})\right)}{\hat{s}_{\mathrm{w}}\left(\chi_{h}(\boldsymbol{\beta})\right)}\right\rangle_{\kappa_{\text {abs }}}-\tau\left\langle h_{\text {ext }}^{n}, \frac{\hat{p}_{\text {pore }}\left(\chi_{h}(\boldsymbol{\beta})\right)}{\hat{s}_{\mathrm{w}}\left(\chi_{h}(\boldsymbol{\beta})\right)}\right\rangle \\
= & : T_{1}+T_{2}+T_{3}+T_{4}
\end{aligned}
$$

where we dropped the explicit dependence of $\boldsymbol{\alpha}$ on $\boldsymbol{\beta}$ and used the identity

$$
\sum_{j=1}^{d_{\mathrm{Q}}} \beta_{j} q_{h, j}=\frac{\hat{p}_{\text {pore }}\left(\chi_{h}(\boldsymbol{\beta})\right)}{\hat{s}_{\mathrm{w}}\left(\chi_{h}(\boldsymbol{\beta})\right)}
$$

We discuss the terms $T_{1}, T_{2}, T_{3}, T_{4}$ separately.

Discussion of $T_{1}$. Let $\bar{b}_{\eta}:=\hat{b}_{\eta} \circ\left(\frac{\hat{p}_{\text {pore }}}{\hat{s}_{\mathrm{w}}}\right)^{-1}$, the energy $\bar{B}_{\eta}$ as in $(4.3)$, and $\boldsymbol{\beta}^{n-1} \in \mathbb{R}^{d_{Q}}$ such that $\chi_{h}^{n-1}=$ $\chi_{h}\left(\boldsymbol{\beta}^{n-1}\right)$. Using Lemma B.12, we obtain

$$
T_{1} \geq\left\|\bar{B}_{\eta}\left(\frac{\hat{p}_{\text {pore }}\left(\chi_{h}(\boldsymbol{\beta})\right)}{\hat{s}_{\mathrm{w}}\left(\chi_{h}(\boldsymbol{\beta})\right)}\right)\right\|_{L^{1}(\Omega)}-\left\|\bar{B}_{\eta}\left(\frac{\hat{p}_{\text {pore }}\left(\chi_{h}\left(\boldsymbol{\beta}^{n-1}\right)\right)}{\hat{s}_{\mathrm{w}}\left(\chi_{h}\left(\boldsymbol{\beta}^{n-1}\right)\right)}\right)\right\|_{L^{1}(\Omega)} .
$$


Discussion of $T_{2}$. The coupling term $T_{2}$ can be reformulated and estimated by employing (5.7) tested with $\boldsymbol{v}_{h}=\boldsymbol{u}_{h}(\boldsymbol{\alpha})-\boldsymbol{u}_{h}^{n-1}$. Under the use of the binomial identity (App. B.2), the Cauchy-Schwarz inequality and Young's inequality, the coupling term $T_{2}$ can be bounded as

$$
T_{2} \geq \zeta \tau^{-1}\left\|\boldsymbol{u}_{h}(\boldsymbol{\alpha})-\boldsymbol{u}_{h}^{n-1}\right\|_{\boldsymbol{V}}^{2}+\frac{1}{2}\left\|\boldsymbol{u}_{h}(\boldsymbol{\alpha})\right\|_{\boldsymbol{V}}^{2}+\frac{1}{4}\left\|\boldsymbol{u}_{h}(\boldsymbol{\alpha})-\boldsymbol{u}_{h}^{n-1}\right\|_{\boldsymbol{V}}^{2}-\frac{1}{2}\left\|\boldsymbol{u}_{h}^{n-1}\right\|_{\boldsymbol{V}}^{2}-\left\|\boldsymbol{f}^{n}\right\|_{\boldsymbol{V}^{\star}}^{2}
$$

Discussion of $T_{3}$. By the mean value theorem and (A4), the diffusion term $T_{3}$ can be estimated from below

$$
T_{3} \geq c_{\hat{p}_{\text {pore }} / \hat{s}_{\mathrm{w}}} \tau\left\|\chi_{h}(\boldsymbol{\beta})\right\|_{1, \mathcal{T}, \kappa_{\mathrm{abs}}}^{2} .
$$

Here, the specific definition of the two-point flux approximation is crucial.

Discussion of $T_{4}$. Employing the definition of $h_{\mathrm{ext}}=\left(h, w_{\mathrm{N}}\right)$, the non-degeneracy condition (ND1), a discrete trace inequality (introducing $C_{\mathrm{tr}}$ ), $c f$. ., Lemma B.2, together with a discrete Poincaré inequality (introducing $\left.C_{\Omega, \mathrm{DP}}\right), c f .$, Lemma B.1, we obtain

$$
\begin{aligned}
\left\langle h_{\text {ext }}^{n}, \frac{\hat{p}_{\text {pore }}\left(\chi_{h}(\boldsymbol{\beta})\right)}{\hat{s}_{\mathrm{w}}\left(\chi_{h}(\boldsymbol{\beta})\right)}\right\rangle & \leq\left\|\frac{\hat{p}_{\text {pore }}\left(\chi_{h}(\boldsymbol{\beta})\right)}{\hat{s}_{\mathrm{w}}\left(\chi_{h}(\boldsymbol{\beta})\right) \chi_{h}(\boldsymbol{\beta})}\right\|_{\infty}\left(\left\|h^{n}\right\|_{L^{2}(\Omega)}\left\|\chi_{h}(\boldsymbol{\beta})\right\|_{L^{2}(\Omega)}+\left\|w_{\mathrm{N}}^{n}\right\|_{L^{2}\left(\Gamma_{\mathrm{N}}^{\mathrm{f}}\right)}\left\|\chi_{h}(\boldsymbol{\beta})\right\|_{L^{2}\left(\Gamma_{\mathrm{N}}^{\mathrm{f}}\right)}\right) \\
& \leq C\left(C_{\mathrm{ND}, 1}, C_{\mathrm{tr}}, C_{\Omega, \mathrm{DP}}\right)\left(\left\|h^{n}\right\|_{L^{2}(\Omega)}+\left\|w_{\mathrm{N}}^{n}\right\|_{L^{2}\left(\Gamma_{\mathrm{N}}^{\mathrm{f}}\right)}\right)\left\|\chi_{h}(\boldsymbol{\beta})\right\|_{1, \mathcal{T}}
\end{aligned}
$$

for a constant $C\left(C_{\mathrm{ND}, 1}, C_{\mathrm{tr}}, C_{\Omega, \mathrm{DP}}\right)>0$. Hence, by (A6) and Young's inequality, for the term $T_{4}$ it holds that

$$
T_{4} \leq \frac{C\left(C_{\mathrm{ND}, 1}, C_{\mathrm{tr}}, C_{\Omega, \mathrm{DP}}\right)^{2}}{2 c_{\hat{p}_{\mathrm{pore}} / \hat{s}_{\mathrm{w}}} \kappa_{\mathrm{m}, \mathrm{abs}}} \tau\left(\left\|h^{n}\right\|_{L^{2}(\Omega)}+\left\|w_{\mathrm{N}}^{n}\right\|_{L^{2}\left(\Gamma_{\mathrm{N}}^{\mathrm{f}}\right)}\right)^{2}+\frac{c_{\hat{p}_{\mathrm{pore}} / \hat{s}_{\mathrm{w}}}}{2} \tau\left\|\chi_{h}(\boldsymbol{\beta})\right\|_{1, \mathcal{T}, \kappa_{\mathrm{abs}}}^{2} .
$$

Combination of all results. By inserting the estimates for $T_{1}, T_{2}, T_{3}$, and $T_{4},(5.9)$ becomes

$$
\begin{aligned}
\langle\boldsymbol{F}(\boldsymbol{\beta}), \boldsymbol{\beta}\rangle \geq & \left(\left\|\bar{B}_{\eta}\left(\frac{\hat{p}_{\text {pore }}\left(\chi_{h}(\boldsymbol{\beta})\right)}{\hat{s}_{\mathrm{w}}\left(\chi_{h}(\boldsymbol{\beta})\right)}\right)\right\|_{L^{1}(\Omega)}+\frac{c_{\hat{p}_{\text {pore }} / \hat{s}_{\mathrm{W}}}}{2} \tau\left\|_{h}(\boldsymbol{\beta})\right\|_{1, \mathcal{T}, \kappa_{\mathrm{abs}}}^{2}\right. \\
& \left.+\frac{1}{4}\left\|\boldsymbol{u}_{h}(\boldsymbol{\alpha})\right\|_{\boldsymbol{V}}^{2}+\left(\zeta \tau^{-1}+\frac{1}{4}\right)\left\|\boldsymbol{u}_{h}(\boldsymbol{\alpha})-\boldsymbol{u}_{h}^{n-1}\right\|_{\boldsymbol{V}}^{2}\right) \\
& -\left(\left\|\bar{B}_{\eta}\left(\frac{\hat{p}_{\text {pore }}\left(\chi_{h}^{n-1}\right)}{\hat{s}_{\mathrm{w}}\left(\chi_{h}^{n-1}\right)}\right)\right\|_{L^{1}(\Omega)}+\frac{1}{2}\left\|\boldsymbol{u}_{h}^{n-1}\right\|_{\boldsymbol{V}}^{2}\right. \\
& \left.+\frac{5}{4}\left\|\boldsymbol{f}^{n}\right\|_{\boldsymbol{V}^{\star}}^{2}+\frac{C\left(C_{\mathrm{ND}, 1}, C_{\mathrm{tr}}, C_{\Omega, \mathrm{DP}}\right)^{2}}{2 c_{\hat{p}_{\text {pore }} / \hat{s}_{\mathrm{w}}} \kappa_{\mathrm{m}, \mathrm{abs}}} \tau\left(\left\|h^{n}\right\|_{L^{2}(\Omega)}+\left\|w_{\mathrm{N}}^{n}\right\|_{L^{2}\left(\Gamma_{\mathrm{N}}^{\mathrm{f}}\right)}\right)^{2}\right) .
\end{aligned}
$$

Finally, since $\|\cdot\|_{1, \mathcal{T}, \kappa_{\mathrm{abs}}}$ defines a norm on $Q_{h}$ and (5.6) holds by induction for $n-1$ if $n \geq 2$ or from (A8) $\eta$ for $n=1$, by Lemma B.4 (a corollary of Brouwer's fixed point theorem), there exists a $\boldsymbol{\beta} \in \mathbb{R}^{d_{Q}}$ such that $\boldsymbol{F}(\boldsymbol{\beta})=\mathbf{0}$. This implies the existence of a discrete solution. The bound (5.6) for $n$ follows immediately from (5.10) due to (A7).

\section{SteP 3: Limit $h, \tau \rightarrow 0$}

In the following, we show that the fully-discrete FEM-TPFA discretization, introduced in the previous section, converges (up to subsequence) to a weak solution of the doubly regularized unsaturated poroelasticity model, i.e., we prove Lemma 4.3. For this, we employ standard compactness arguments. Throughout the entire section, we assume (A0)-(A8) and (ND1) hold true. 


\subsection{Stability estimates for the fully-discrete approximation}

Lemma 6.1 (Stability estimate for the primary variables). Let $\tau<\frac{1}{8}$. There exists a constant $C^{(1)}>0$ (independent of $h, \tau, \zeta, \eta$ ), such that

$$
\begin{aligned}
& \zeta \sum_{n=1}^{N} \tau^{-1}\left\|\boldsymbol{u}_{h}^{n}-\boldsymbol{u}_{h}^{n-1}\right\|_{\boldsymbol{V}}^{2}+\sup _{n}\left\|\boldsymbol{u}_{h}^{n}\right\|_{\boldsymbol{V}}^{2}+\sum_{n=1}^{N}\left\|\boldsymbol{u}_{h}^{n}-\boldsymbol{u}_{h}^{n-1}\right\|_{\boldsymbol{V}}^{2}+\sum_{n=1}^{N} \tau\left\|_{h}^{n}\right\|_{1, \mathcal{T}}^{2} \\
& \quad \leq C^{(1)}\left(C_{0}, C_{\mathrm{ND}, 1},\left\|h_{\mathrm{ext}}\right\|_{L^{2}\left(0, T ; Q^{\star}\right)},\left\|\boldsymbol{f}_{\mathrm{ext}}\right\|_{H^{1}\left(0, T ; \boldsymbol{V}^{\star}\right)}\right)
\end{aligned}
$$

where $C_{0}$ and $C_{\mathrm{ND}, 1}$ are defined in $\left(\mathrm{A} 8^{\star}\right)_{h}$ and $(\mathrm{ND} 1)$, respectively.

Proof. The proof follows essentially the same steps as the proof of Lemma 5.6. Therefore, we are brief on similar steps. We consider the reduced displacement-pressure formulation (5.5). We choose $\boldsymbol{v}_{h}=\boldsymbol{u}_{h}^{n}-\boldsymbol{u}_{h}^{n-1}$ and $q_{h}=\frac{\hat{p}_{\text {pore }}\left(\chi_{h}^{n}\right)}{\hat{s}_{\mathrm{w}}\left(\chi_{h}^{n}\right)}$ as test functions and sum the two equations; note that the second is well-defined as $\hat{s}_{\mathrm{W}}(\chi)>0$ for all $\chi \in \mathbb{R}$, by (A2). We obtain

$$
\begin{aligned}
& \zeta \tau^{-1}\left\|\boldsymbol{u}_{h}^{n}-\boldsymbol{u}_{h}^{n-1}\right\|_{\boldsymbol{V}}^{2}+a\left(\boldsymbol{u}_{h}^{n}, \boldsymbol{u}_{h}^{n}-\boldsymbol{u}_{h}^{n-1}\right)+\left\langle\hat{b}_{\eta}\left(\chi_{h}^{n}\right)-\hat{b}_{\eta}\left(\chi_{h}^{n-1}\right), \frac{\hat{p}_{\text {pore }}\left(\chi_{h}^{n}\right)}{\hat{s}_{\mathrm{w}}\left(\chi_{h}^{n}\right)}\right\rangle+\tau\left\langle\boldsymbol{\nabla}_{h} \chi_{h}^{n}, \boldsymbol{\nabla}_{h} \frac{\hat{p}_{\text {pore }}\left(\chi_{h}^{n}\right)}{\hat{s}_{\mathrm{w}}\left(\chi_{h}^{n}\right)}\right\rangle_{\kappa_{\mathrm{abs}}} \\
& =\left\langle\boldsymbol{f}_{\mathrm{ext}}^{n}, \boldsymbol{u}_{h}^{n}-\boldsymbol{u}_{h}^{n-1}\right\rangle+\tau\left\langle h_{\mathrm{ext}}^{n}, \frac{\hat{p}_{\text {pore }}\left(\chi_{h}^{n}\right)}{\hat{s}_{\mathrm{w}}\left(\chi_{h}^{n}\right)}\right\rangle
\end{aligned}
$$

On the left hand side, we employ the binomial identity (App. B.2), Lemma B.12 for the energy term $\bar{B}_{\eta}$ defined in (4.3), and the uniform increase of $\frac{\hat{p}_{\text {pore }}}{\hat{s}_{\mathrm{w}}}, c f .,(\mathrm{A} 4)$. It holds that

$$
\begin{aligned}
& \zeta \tau^{-1}\left\|\boldsymbol{u}_{h}^{n}-\boldsymbol{u}_{h}^{n-1}\right\|_{\boldsymbol{V}}^{2}+\frac{1}{2}\left(\left\|\boldsymbol{u}_{h}^{n}\right\|_{\boldsymbol{V}}^{2}-\left\|\boldsymbol{u}_{h}^{n-1}\right\|_{\boldsymbol{V}}^{2}+\left\|\boldsymbol{u}_{h}^{n}-\boldsymbol{u}_{h}^{n-1}\right\|_{\boldsymbol{V}}^{2}\right) \\
& \quad+\left\|\bar{B}_{\eta}\left(\frac{\hat{p}_{\text {pore }}\left(\chi_{h}^{n}\right)}{\hat{s}_{\mathrm{w}}\left(\chi_{h}^{n}\right)}\right)\right\|_{L^{1}(\Omega)}-\left\|\bar{B}_{\eta}\left(\frac{\hat{p}_{\text {pore }}\left(\chi_{h}^{n-1}\right)}{\hat{s}_{\mathrm{w}}\left(\chi_{h}^{n-1}\right)}\right)\right\|_{L^{1}(\Omega)} \\
& \quad+c_{\hat{p}_{\text {pore }} / \hat{s}_{\mathrm{w}}} \tau\left\|\chi_{h}^{n}\right\|_{1, \mathcal{T}, \kappa_{\mathrm{abs}}}^{2} \\
& \quad \leq\left\langle\boldsymbol{f}_{\mathrm{ext}}^{n}, \boldsymbol{u}_{h}^{n}-\boldsymbol{u}_{h}^{n-1}\right\rangle+\left\langle h_{\mathrm{ext}}^{n}, \frac{\hat{p}_{\mathrm{pore}}\left(\chi_{h}^{n}\right)}{\hat{s}_{\mathrm{w}}\left(\chi_{h}^{n}\right)}\right\rangle
\end{aligned}
$$

Later on, we sum over the time steps 1 to $N$ and utilize a telescope sum. Let us first separately discuss the sums of the two terms on the right hand side. For the first of them, we employ summation by parts, $c f$. ., Lemma B.6, as well as the Cauchy-Schwarz inequality and Young's inequality. We obtain

$$
\begin{aligned}
& \sum_{n=1}^{N}\left\langle\boldsymbol{f}_{\mathrm{ext}}^{n}, \boldsymbol{u}_{h}^{n}-\boldsymbol{u}_{h}^{n-1}\right\rangle \\
& \quad=\left\langle\boldsymbol{f}_{\mathrm{ext}}^{N}, \boldsymbol{u}_{h}^{N}\right\rangle-\left\langle\boldsymbol{f}_{\mathrm{ext}}^{1}, \boldsymbol{u}_{h}^{0}\right\rangle-\sum_{n=1}^{N-1}\left\langle\boldsymbol{f}_{\mathrm{ext}}^{n+1}-\boldsymbol{f}_{\mathrm{ext}}^{n}, \boldsymbol{u}_{h}^{n}\right\rangle \\
& \quad \leq\left\|\boldsymbol{f}_{\mathrm{ext}}^{N}\right\|_{\boldsymbol{V}^{\star}}^{2}+\frac{1}{4}\left\|\boldsymbol{u}_{h}^{N}\right\|_{\boldsymbol{V}}^{2}+\frac{1}{2}\left\|\boldsymbol{f}_{\mathrm{ext}}^{1}\right\|_{\boldsymbol{V}^{\star}}^{2}+\frac{1}{2}\left\|\boldsymbol{u}_{h}^{0}\right\|_{\boldsymbol{V}}^{2}+\sum_{n=1}^{N} \tau^{-1}\left\|\boldsymbol{f}_{\mathrm{ext}}^{n}-\boldsymbol{f}_{\mathrm{ext}}^{n-1}\right\|_{\boldsymbol{V}^{\star}}^{2}+\sum_{n=1}^{N} \tau\left\|\boldsymbol{u}_{h}^{n}\right\|_{\boldsymbol{V}^{2}}^{2}
\end{aligned}
$$


The second term is estimated as in the discussion of $T_{4}$ within the proof of Lemma 5.6. We obtain

$$
\begin{aligned}
& \sum_{n=1}^{N} \tau\left\langle h_{\text {ext }}^{n}, \frac{\hat{p}_{\text {pore }}\left(\chi_{h}^{n}\right)}{\hat{s}_{\mathrm{w}}\left(\chi_{h}^{n}\right)}\right\rangle \\
& \quad \leq \frac{C\left(C_{\mathrm{ND}, 1}, C_{\mathrm{tr}}, C_{\Omega, \mathrm{DP}}\right)^{2}}{2 c_{\hat{p}_{\text {pore }} / \hat{s}_{\mathrm{w}}} \kappa_{\mathrm{m}, \mathrm{abs}}} \sum_{n=1}^{N} \tau\left(\left\|h^{n}\right\|_{L^{2}(\Omega)}+\left\|w_{\mathrm{N}}^{n}\right\|_{L^{2}\left(\Gamma_{\mathrm{N}}^{\mathrm{f}}\right)}\right)^{2}+\frac{c_{\hat{p}_{\text {pore }} / \hat{s}_{\mathrm{w}}}}{2} \sum_{n=1}^{N} \tau\left\|\chi_{h}^{n}\right\|_{1, \mathcal{T}, \kappa_{\mathrm{abs}}}^{2} .
\end{aligned}
$$

Summing (6.1) over time steps 1 to $N$, inserting (6.2) and (6.3), and rearranging terms, yields

$$
\begin{aligned}
& \zeta \sum_{n=1}^{N} \tau^{-1}\left\|\boldsymbol{u}_{h}^{n}-\boldsymbol{u}_{h}^{n-1}\right\|_{\boldsymbol{V}}^{2}+\frac{1}{4}\left\|\boldsymbol{u}_{h}^{N}\right\|_{\boldsymbol{V}}^{2}+\frac{1}{4} \sum_{n=1}^{N}\left\|\boldsymbol{u}_{h}^{n}-\boldsymbol{u}_{h}^{n-1}\right\|_{\boldsymbol{V}}^{2} \\
& +\left\|\bar{B}_{\eta}\left(\frac{\hat{p}_{\text {pore }}\left(\chi_{h}^{N}\right)}{\hat{s}_{\mathrm{w}}\left(\chi_{h}^{N}\right)}\right)\right\|_{L^{1}(\Omega)}+\frac{c_{\hat{p}_{\text {pore }} / \hat{s}_{\mathrm{w}}}}{2} \sum_{n=1}^{N} \tau\left\|\chi_{h}^{n}\right\|_{1, \mathcal{T}, \kappa_{\mathrm{abs}}}^{2} \\
& \leq\left\|\boldsymbol{u}_{h}^{0}\right\|_{\boldsymbol{V}}^{2}+\left\|\bar{B}_{\eta}\left(\frac{\hat{p}_{\text {pore }}\left(\chi_{h}^{0}\right)}{\hat{s}_{\mathrm{w}}\left(\chi_{h}^{0}\right)}\right)\right\|_{L^{1}(\Omega)} \\
& \quad+\max _{n=0, \ldots, N}\left\|\boldsymbol{f}_{\text {ext }}^{n}\right\|_{\boldsymbol{V}^{\star}}^{2}+\sum_{n=1}^{N} \tau^{-1}\left\|\boldsymbol{f}_{\mathrm{ext}}^{n}-\boldsymbol{f}_{\mathrm{ext}}^{n-1}\right\|_{\boldsymbol{V}^{\star}}^{2}+\sum_{n=1}^{N} \tau\left\|\boldsymbol{f}_{\mathrm{ext}}^{n}\right\|_{\boldsymbol{V}^{\star}}^{2} \\
& \quad+\frac{C\left(C_{\mathrm{ND}, 1}, C_{\mathrm{tr}}, C_{\Omega, \mathrm{DP}}\right)^{2}}{2 c_{\hat{p}_{\text {pore }} / \hat{s}_{\mathrm{w}}} \kappa_{\mathrm{m}, \mathrm{abs}}} \sum_{n=1}^{N} \tau\left(\left\|h^{n}\right\|_{L^{2}(\Omega)}+\left\|w_{\mathrm{N}}^{n}\right\|_{L^{2}\left(\Gamma_{\mathrm{N}}^{\mathrm{f}}\right)}\right)^{2}+2 \sum_{n=1}^{N} \tau\left\|\boldsymbol{u}_{h}^{n}\right\|_{\boldsymbol{V}}^{2} .
\end{aligned}
$$

Finally, the last term on the right hand side can be controlled after applying a discrete Grönwall inequality, $c f$., Lemma B.7, using that $2 \tau<\frac{1}{4}$. The thesis follows from the assumptions on the regularity of the source terms (A7) (together with a Sobolev embedding) and initial data $(\mathrm{A} 8)_{\eta, h}$.

Lemma 6.2 (Stability for the Kirchhoff pressure). There exists a constant $C_{\zeta \eta}^{(2)}>0$ (independent of $h, \tau$ ) such that

$$
b_{\chi, m} \sum_{n=1}^{N} \tau^{-1}\left\|\chi_{h}^{n}-\chi_{h}^{n-1}\right\|_{L^{2}(\Omega)}^{2}+\left\|\chi_{h}^{N}\right\|_{1, \mathcal{T}}^{2}+\sum_{n=1}^{N}\left\|\chi_{h}^{n}-\chi_{h}^{n-1}\right\|_{1, \mathcal{T}}^{2} \leq C_{\zeta \eta}^{(2)}\left(C_{0}, \frac{1+\zeta^{-1}}{b_{\chi, \mathrm{m}}} C^{(1)}\right),
$$

where $b_{\chi, \mathrm{m}}$ is from the growing condition $\left(\mathrm{A} 1^{\star}\right), C^{(1)}$ is the stability constant from Lemma 6.1 , and $C_{0}$ is the bound in $\left(\mathrm{A} 8^{\star}\right)_{h}$.

Proof. We test (5.5b) with $q_{h}=\chi_{h}^{n}-\chi_{h}^{n-1}$. By using the binomial identity (App. B.2), for the diffusion term, we obtain

$$
\begin{aligned}
& \left\langle\hat{b}_{\eta}\left(\chi_{h}^{n}\right)-\hat{b}_{\eta}\left(\chi_{h}^{n-1}\right), \chi_{h}^{n}-\chi_{h}^{n-1}\right\rangle+\alpha\left\langle\hat{s}_{\mathrm{w}}\left(\chi_{h}^{n}\right) \boldsymbol{\nabla} \cdot\left(\boldsymbol{u}_{h}^{n}-\boldsymbol{u}_{h}^{n-1}\right), \chi_{h}^{n}-\chi_{h}^{n-1}\right\rangle \\
& \quad+\frac{\tau}{2}\left(\left\|\chi_{h}^{n}\right\|_{1, \mathcal{T}, \kappa_{\mathrm{abs}}}^{2}-\left\|\chi_{h}^{n-1}\right\|_{1, \mathcal{T}, \kappa_{\mathrm{abs}}}^{2}+\left\|\chi_{h}^{n}-\chi_{h}^{n-1}\right\|_{1, \mathcal{T}, \kappa_{\mathrm{abs}}}^{2}\right)=\tau\left\langle h_{\mathrm{ext}}^{n}, \chi_{h}^{n}-\chi_{h}^{n-1}\right\rangle .
\end{aligned}
$$

Dividing by $\tau$ and summing over time steps 1 to $N$, yields

$$
\begin{aligned}
& \sum_{n=1}^{N} \tau^{-1}\left\langle\hat{b}_{\eta}\left(\chi_{h}^{n}\right)-\hat{b}_{\eta}\left(\chi_{h}^{n-1}\right), \chi_{h}^{n}-\chi_{h}^{n-1}\right\rangle+\frac{1}{2}\left\|\chi_{h}^{N}\right\|_{1, \mathcal{T}, \kappa_{\mathrm{abs}}}^{2}+\frac{1}{2} \sum_{n=1}^{N}\left\|\chi_{h}^{n}-\chi_{h}^{n-1}\right\|_{1, \mathcal{T}, \kappa_{\mathrm{abs}}}^{2} \\
& \quad=\frac{1}{2}\left\|\chi_{h}^{0}\right\|_{1, \mathcal{T}, \kappa_{\mathrm{abs}}}^{2}+\sum_{n=1}^{N}\left\langle h_{\mathrm{ext}}^{n}, \chi_{h}^{n}-\chi_{h}^{n-1}\right\rangle-\alpha \sum_{n=1}^{N} \tau^{-1}\left\langle\hat{s}_{\mathrm{w}}\left(\chi_{h}^{n}\right) \boldsymbol{\nabla} \cdot\left(\boldsymbol{u}_{h}^{n}-\boldsymbol{u}_{h}^{n-1}\right), \chi_{h}^{n}-\chi_{h}^{n-1}\right\rangle .
\end{aligned}
$$


Employing the growth condition $(\mathrm{A} 1)_{\eta}$ for the first term on the left hand side, and the Cauchy-Schwarz inequality and Young's inequality for the last two terms on the right hand side, yields

$$
\begin{aligned}
& \frac{b_{\chi, m}}{4} \sum_{n=1}^{N} \tau^{-1}\left\|\chi_{h}^{n}-\chi_{h}^{n-1}\right\|_{L^{2}(\Omega)}^{2}+\frac{1}{2}\left\|\chi_{h}^{N}\right\|_{1, \mathcal{T}, \kappa_{\mathrm{abs}}}^{2}+\frac{1}{2} \sum_{n=1}^{N}\left\|\chi_{h}^{n}-\chi_{h}^{n-1}\right\|_{1, \mathcal{T}, \kappa_{\mathrm{abs}}}^{2} \\
& \quad \leq \frac{1}{2}\left\|\chi_{h}^{0}\right\|_{1, \mathcal{T}, \kappa_{\mathrm{abs}}}^{2}+\frac{1}{2 b_{\chi, \mathrm{m}}} \sum_{n=1}^{N} \tau\left\|h_{\mathrm{ext}}^{n}\right\|_{Q^{\star}}^{2}+\frac{\alpha^{2}}{b_{\chi, \mathrm{m}}} \sum_{n=1}^{N} \tau^{-1}\left\|\nabla \cdot\left(\boldsymbol{u}_{h}^{n}-\boldsymbol{u}_{h}^{n-1}\right)\right\|_{L^{2}(\Omega)}^{2}
\end{aligned}
$$

Finally, the first two terms on the right hand side are bounded by $\left(\mathrm{A} 8^{\star}\right)_{h}$ and $(\mathrm{A} 7)$, whereas the last term can be bounded by Lemma 6.1. On the left hand side, we employ (A6).

Lemma 6.3 (Stability for the energy term $\hat{B}_{\eta}$ ). Let $\hat{B}_{\eta}(z)$ denote the energy term based on $\hat{b}$ defined in (4.2). There exists a constant $C_{\zeta}^{(3)}>0$ (independent of $h, \tau, \eta$ ), such that

$$
\max _{n=0, \ldots, N}\left\|\hat{B}_{\eta}\left(\chi_{h}^{n}\right)\right\|_{L^{1}(\Omega)} \leq C_{\zeta}^{(3)}\left(C_{0}, C^{(1)}\left(1+\zeta^{-1}\right)\right)
$$

where $C_{0}$ and $C^{(1)}$ are the constants from $\left(\mathrm{A}^{\star}\right)_{h}$ and Lemma 6.1, respectively.

Proof. Testing (5.5b) with $q_{h}=\chi_{h}^{n}$ and employing Lemma B.12, yields for all $n$

$$
\left\|\hat{B}_{\eta}\left(\chi_{h}^{n}\right)\right\|_{L^{1}(\Omega)}-\left\|\hat{B}_{\eta}\left(\chi_{h}^{n-1}\right)\right\|_{L^{1}(\Omega)}+\tau\left\|\chi_{h}^{n}\right\|_{1, \mathcal{T}, \kappa_{\mathrm{abs}}}^{2} \leq \tau\left\langle h_{\mathrm{ext}}^{n}, \chi_{h}^{n}\right\rangle-\alpha\left\langle\hat{s}_{\mathrm{w}}\left(\chi_{h}^{n}\right) \nabla \cdot\left(\boldsymbol{u}_{h}^{n}-\boldsymbol{u}_{h}^{n-1}\right), \chi_{h}^{n}\right\rangle .
$$

For the first term on the right hand side, we employ a similar bound as in the discussion of $T_{4}$ within the proof of Lemma 5.6. For the second term, we employ the Cauchy-Schwarz inequality, a discrete Poincaré inequality (introducing $C_{\Omega, \mathrm{DP}}$ ), and (A6). We obtain

$$
\begin{aligned}
& \left\|\hat{B}_{\eta}\left(\chi_{h}^{n}\right)\right\|_{L^{1}(\Omega)}-\left\|\hat{B}_{\eta}\left(\chi_{h}^{n-1}\right)\right\|_{L^{1}(\Omega)}+\frac{\tau}{2}\left\|\chi_{h}^{n}\right\|_{1, \mathcal{T}, \kappa_{\mathrm{abs}}}^{2} \\
& \quad \leq \frac{C\left(C_{\mathrm{ND}, 1}, C_{\mathrm{tr}}, C_{\Omega, \mathrm{DP}}\right)^{2}}{\kappa_{\mathrm{m}, \mathrm{abs}}} \tau\left\|h_{\mathrm{ext}}^{n}\right\|_{Q^{\star}}^{2}+\frac{C_{\Omega, \mathrm{DP}}}{\kappa_{\mathrm{m}, \mathrm{abs}}} \frac{\alpha^{2}}{K_{\mathrm{dr}}} \tau^{-1}\left\|\boldsymbol{u}_{h}^{n}-\boldsymbol{u}_{h}^{n-1}\right\|_{\boldsymbol{V}^{2}}^{2}
\end{aligned}
$$

Finally, summing over time steps 1 to $N$ and employing Lemma 6.1 and (A7) proves the assertion.

Lemma 6.4 (Stability for the pore pressure). There exists a constant $C^{(4)}>0$ (independent of $h, \tau, \zeta, \eta$ ), such that

$$
\sum_{n=1}^{N} \tau\left\|\hat{p}_{\text {pore }}\left(\chi_{h}^{n}\right)\right\|_{L^{2}(\Omega)}^{2} \leq C^{(4)}\left(C^{(1)}\right),
$$

where $C^{(1)}$ is the stability constant from Lemma 6.1.

Proof. We utilize a standard inf-sup argument (introducing $C_{\Omega \text {,is }}$ ), $c f$., Lemma B.11. Due to (D2), there exists a $\boldsymbol{v}_{h} \in \boldsymbol{V}_{h}$ such that

$$
\begin{aligned}
\left\|\hat{p}_{\text {pore }}\left(\chi_{h}^{n}\right)\right\|_{L^{2}(\Omega)}^{2} & =\alpha\left\langle\hat{p}_{\text {pore }}\left(\chi_{h}^{n}\right), \nabla \cdot \boldsymbol{v}_{h}\right\rangle, \text { and } \\
\left\|\boldsymbol{v}_{h}\right\|_{\boldsymbol{V}} & \leq C_{\Omega, \text { is }}\left\|\hat{p}_{\text {pore }}\left(\chi_{h}^{n}\right)\right\|_{L^{2}(\Omega)},
\end{aligned}
$$

Employing the mechanics equation (4.4a), we obtain

$$
\left\|\hat{p}_{\text {pore }}\left(\chi_{h}^{n}\right)\right\|_{L^{2}(\Omega)} \leq C_{\Omega, \text { is }}\left(\zeta \tau^{-1}\left\|\boldsymbol{u}_{h}^{n}-\boldsymbol{u}_{h}^{n-1}\right\|_{\boldsymbol{V}}+\left\|\boldsymbol{u}_{h}^{n}\right\|_{\boldsymbol{V}}+\left\|\boldsymbol{f}_{\mathrm{ext}}^{n}\right\|_{\boldsymbol{V}^{\star}}\right)
$$


and hence,

$$
\sum_{n=1}^{N} \tau\left\|\hat{p}_{\text {pore }}\left(\chi_{h}^{n}\right)\right\|_{L^{2}(\Omega)}^{2} \leq 3 C_{\Omega, \text { is }}^{2}\left(\zeta^{2} \sum_{n=1}^{N} \tau^{-1}\left\|\boldsymbol{u}_{h}^{n}-\boldsymbol{u}_{h}^{n-1}\right\|_{\boldsymbol{V}}^{2}+\sum_{n=1}^{N} \tau\left\|\boldsymbol{u}_{h}^{n}\right\|_{\boldsymbol{V}}^{2}+\sum_{n=1}^{N} \tau\left\|\boldsymbol{f}_{\mathrm{ext}}^{n}\right\|_{\boldsymbol{V}^{\star}}^{2}\right) .
$$

Finally, the assertion follows from Lemma 6.1, assuming wlog. $\zeta$ is bounded from above.

Lemma 6.5 (Stability for the temporal change of $\hat{b}$ ). There exists a constant $C_{\zeta}^{(5)}>0$ (independent of $\left.h, \tau, \eta\right)$, such that

$$
\sup _{\left\{q_{h}^{n}\right\}_{n} \subset Q_{h} \backslash\{0\}} \frac{\sum_{n=1}^{N} \tau\left\langle\frac{\hat{b}_{\eta}\left(\chi_{h}^{n}\right)-\hat{b}_{\eta}\left(\chi_{h}^{n-1}\right)}{\tau}, q_{h}^{n}\right\rangle}{\left(\sum_{n=1}^{N} \tau\left\|q_{h}^{n}\right\|_{1, \mathcal{T}}^{2}\right)^{1 / 2}} \leq C_{\zeta}^{(5)}\left(C^{(1)}\left(1+\zeta^{-1}\right)\right),
$$

where $C^{(1)}$ is the stability constant from Lemma 6.1.

Proof. Let $\left\{q_{h}^{n}\right\}_{n} \subset Q_{h} \backslash\{0\}$ be an arbitrary sequence of test functions. Employ $q_{h}^{n}$ as test function in (5.5b). Summing over time steps 1 to $N$ and applying the Cauchy-Schwarz inequality, yields

$$
\begin{aligned}
\sum_{n=1}^{N} \tau\left\langle\frac{\hat{b}_{\eta}\left(\chi_{h}^{n}\right)-\hat{b}_{\eta}\left(\chi_{h}^{n-1}\right)}{\tau}, q_{h}^{n}\right\rangle \leq & \left(\frac{\alpha^{2}}{K_{\mathrm{dr}}} \sum_{n=1}^{N} \tau^{-1}\left\|\boldsymbol{u}_{h}^{n}-\boldsymbol{u}_{h}^{n-1}\right\|_{\boldsymbol{V}}^{2}\right)^{1 / 2}\left(\sum_{n=1}^{N} \tau\left\|q_{h}^{n}\right\|_{L^{2}(\Omega)}^{2}\right)^{1 / 2} \\
& +\left(\sum_{n=1}^{N} \tau\left\|\chi_{h}^{n}\right\|_{1, \mathcal{T}, \kappa_{\mathrm{abs}}}^{2}\right)^{1 / 2}\left(\sum_{n=1}^{N} \tau\left\|q_{h}^{n}\right\|_{1, \mathcal{T}, \kappa_{\mathrm{abs}}}^{2}\right)^{1 / 2} \\
& +\left(1+C_{\mathrm{tr}}\right) C_{\Omega, \mathrm{DP}}\left(\sum_{n=1}^{N} \tau\left\|h_{\mathrm{ext}}^{n}\right\|_{Q^{\star}}^{2}\right)^{1 / 2}\left(\sum_{n=1}^{N} \tau\left\|q_{h}^{n}\right\|_{1, \mathcal{T}}^{2}\right)^{1 / 2} .
\end{aligned}
$$

For the last term, we employed a discrete trace inequality (introducing $C_{\mathrm{tr}}$ ), $c f$., Lemma B.2, and a discrete Poincaré inequality (introducing $C_{\Omega, \mathrm{DP}}$ ), cf., Lemma B.1. Finally, after utilizing a discrete Poincaré inequality for the first term on the right hand side, (A6) on the second term, the assertion follows by Lemma 6.1 with $C_{\zeta}^{(5)}:=\sqrt{C^{(1)}}\left(C_{\Omega, \mathrm{DP}} \frac{\alpha}{\zeta^{1 / 2} K_{\mathrm{dr}}^{1 / 2}}+\kappa_{\mathrm{M}, \mathrm{abs}}^{1 / 2}+\left(1+C_{\mathrm{tr}}\right) C_{\Omega, \mathrm{DP}}\right)$.

\subsection{Stability estimates for interpolants in time}

Utilizing the discrete-in-time approximations $\left(\boldsymbol{u}_{h}^{n}, \chi_{h}^{n}\right)_{n}$, defined by (5.5), we define continuous-in-time approximations on $(0, T]$ by piecewise constant interpolation

$$
\begin{array}{ll}
\overline{\boldsymbol{u}}_{h \tau}(t):=\boldsymbol{u}_{h}^{n}, & t \in\left(t_{n-1}, t_{n}\right], \\
\bar{\chi}_{h \tau}(t):=\chi_{h}^{n}, & t \in\left(t_{n-1}, t_{n}\right],
\end{array}
$$

and by piecewise linear interpolation

$$
\begin{array}{ll}
\hat{\boldsymbol{u}}_{h \tau}(t):=\boldsymbol{u}_{h}^{n-1}+\frac{t-t_{n-1}}{\tau}\left(\boldsymbol{u}_{h}^{n}-\boldsymbol{u}_{h}^{n-1}\right), & t \in\left(t_{n-1}, t_{n}\right], \\
\hat{\chi}_{h \tau}(t):=\chi_{h}^{n-1}+\frac{t-t_{n-1}}{\tau}\left(\chi_{h}^{n}-\chi_{h}^{n-1}\right), & t \in\left(t_{n-1}, t_{n}\right] .
\end{array}
$$

We deduce stability for the interpolants from the stability of the fully discrete approximation. 
Lemma 6.6 (Stability estimate for time interpolants of the mechanical displacement). For all $h, \tau>0$ and $\hat{\tau} \in[0, \tau)$ it holds that

$$
\begin{aligned}
\zeta \int_{0}^{T}\left\|\partial_{t} \hat{\boldsymbol{u}}_{h \tau}\right\|_{\boldsymbol{V}}^{2} \mathrm{~d} t+\left\|\overline{\boldsymbol{u}}_{h \tau}\right\|_{L^{\infty}(0, T ; \boldsymbol{V})}^{2} \leq C^{(1)}, \\
\int_{0}^{T-\hat{\tau}}\left\|\overline{\boldsymbol{u}}_{h \tau}(t+\hat{\tau})-\overline{\boldsymbol{u}}_{h \tau}(t)\right\|_{\boldsymbol{V}}^{2} \mathrm{~d} t \leq C^{(1)} \hat{\tau}, \\
\left\|\overline{\boldsymbol{u}}_{h \tau}-\hat{\boldsymbol{u}}_{h \tau}\right\|_{L^{2}\left(Q_{T}\right)}^{2} \leq C^{(1)} \tau,
\end{aligned}
$$

where $C^{(1)}$ is the stability constant from Lemma 6.1.

Proof. Application of the definition of the interpolation operators and simple calculations yield

$$
\begin{aligned}
& \zeta \int_{0}^{T}\left\|\partial_{t} \hat{\boldsymbol{u}}_{h \tau}\right\|_{\boldsymbol{V}}^{2} \mathrm{~d} t+\left\|\overline{\boldsymbol{u}}_{h \tau}\right\|_{L^{\infty}(0, T ; \boldsymbol{V})}^{2}=\zeta \sum_{n=1}^{N}\left\|\boldsymbol{u}_{h}^{n}-\boldsymbol{u}_{h}^{n-1}\right\|_{\boldsymbol{V}}^{2}+\max _{n=0, \ldots, N}\left\|\boldsymbol{u}_{h}^{n}\right\|_{\boldsymbol{V}}^{2}, \\
& \int_{0}^{T-\hat{\tau}}\left\|\overline{\boldsymbol{u}}_{h \tau}(t+\hat{\tau})-\overline{\boldsymbol{u}}_{h \tau}(t)\right\|_{\boldsymbol{V}}^{2} \mathrm{~d} t=\hat{\tau} \sum_{n=1}^{N}\left\|\boldsymbol{u}_{h}^{n}-\boldsymbol{u}_{h}^{n-1}\right\|_{\boldsymbol{V}}^{2} \\
&\left\|\overline{\boldsymbol{u}}_{h \tau}-\hat{\boldsymbol{u}}_{h \tau}\right\|_{L^{2}\left(Q_{T}\right)}^{2}=\frac{1}{3} \tau \sum_{n=1}^{N}\left\|\boldsymbol{u}_{h}^{n}-\boldsymbol{u}_{h}^{n-1}\right\|_{L^{2}(\Omega)}^{2} .
\end{aligned}
$$

Hence, the assertion follows directly from Lemma 6.1 .

Analogously, we conclude stability for the interpolants of the Kirchhoff pressure.

Lemma 6.7 (Stability estimate for time interpolants of the Kirchhoff pressure). For all $h, \tau>0$ and $\hat{\tau} \in[0, \tau)$ it holds that

$$
\begin{aligned}
\int_{0}^{T}\left\|\bar{\chi}_{h \tau}(t)\right\|_{1, \mathcal{T}}^{2} \mathrm{~d} t & \leq C^{(1)}, \\
b_{\chi, \mathrm{m}}\left\|\partial_{t} \hat{\chi}_{h \tau}\right\|_{L^{2}\left(Q_{T}\right)}^{2}+\left\|\bar{\chi}_{h \tau}\right\|_{L^{\infty}\left(0, T ; L^{2}(\Omega)\right)}^{2} & \leq C_{\zeta \eta}^{(2)}, \\
\int_{0}^{T-\hat{\tau}}\left\|\bar{\chi}_{h \tau}(t+\hat{\tau})-\bar{\chi}_{h \tau}(t)\right\|_{L^{2}(\Omega)}^{2} \mathrm{~d} t & \leq C_{\Omega, \mathrm{DP}}^{2} C_{\zeta \eta}^{(2)} \hat{\tau}, \\
\left\|\bar{\chi}_{h \tau}-\hat{\chi}_{h \tau}\right\|_{L^{2}\left(Q_{T}\right)}^{2} & \leq C_{\Omega, \mathrm{DP}}^{2} C_{\zeta \eta}^{(2)} \tau,
\end{aligned}
$$

where $C^{(1)}$ and $C_{\zeta \eta}^{(2)}$ are the stability constants from Lemmas 6.1 and 6.2, respectively, and $C_{\Omega, \mathrm{DP}}$ is the discrete Poincaré constant, cf., Lemma B.1.

Proof. The proof is analogous to the proof Lemma 6.6. For (6.6c) and (6.6d), a discrete Poincaré inequality, cf., Lemma B.1, has to be applied before utilizing the stability bound on $\sum_{n=1}^{N}\left\|\chi_{h}^{n}-\chi_{h}^{n-1}\right\|_{1, \mathcal{T}}^{2}$ from Lemma 6.2.

Similarly, by definition of the piecewise constant interpolation, we deduce stability for some of the nonlinearities used in the model.

Lemma 6.8 (Stability estimates for non-linearities evaluated in interpolants). It holds that

$$
\begin{aligned}
\left\|\hat{B}_{\eta}\left(\bar{\chi}_{h \tau}\right)\right\|_{L^{\infty}\left(0, T ; L^{1}(\Omega)\right)} & \leq C_{\zeta}^{(3)}, \\
\left\|\hat{p}_{\text {pore }}\left(\bar{\chi}_{h \tau}\right)\right\|_{L^{2}\left(Q_{T}\right)}^{2} & \leq C^{(4)},
\end{aligned}
$$

where $C_{\zeta}^{(3)}$ and $C^{(4)}$ are the bounds from Lemmas 6.3 and 6.4, resp. 
Lemma 6.9 (Stability estimate for the temporal change of $\hat{b}$ ). Let

$$
\bar{\lambda}_{h \tau}(t):=\frac{\hat{b}_{\eta}\left(\chi_{h}^{n}\right)-\hat{b}_{\eta}\left(\chi_{h}^{n-1}\right)}{\tau}, \quad t \in\left(t_{n-1}, t_{n}\right], n \geq 1 .
$$

It holds that

$$
\left\|\bar{\lambda}_{h \tau}\right\|_{L^{2}\left(0, T ; H^{-1}(\Omega)\right)} \leq C_{\Omega, P}^{1 / 2} C_{\zeta}^{(5)}
$$

where $C_{\zeta}^{(5)}$ is the bound from Lemma 6.5, and $C_{\Omega, \mathrm{P}}$ is a Poincaré constant.

Proof. Let $q \in L^{2}(0, T ; Q)$. We define a piecewise constant interpolation in both space and time, and only time. For $n \geq 1$, let

$$
\begin{array}{ll}
q_{h}^{n}(x, t):=\frac{1}{\tau} \int_{t_{n-1}}^{t_{n}} \frac{1}{|K|} \int_{K} q \mathrm{~d} x \mathrm{~d} t, & (x, t) \in K \times\left(t_{n-1}, t_{n}\right], K \in \mathcal{T}, \\
q^{n}(x, t):=\frac{1}{\tau} \int_{t_{n-1}}^{t_{n}} q \mathrm{~d} t, & (x, t) \in \Omega \times\left(t_{n-1}, t_{n}\right] .
\end{array}
$$

Then by Lemma 6.5 it holds that

$$
\int_{0}^{T}\left\langle\bar{\lambda}_{h \tau}, q\right\rangle=\sum_{n=1}^{N} \tau\left\langle\frac{\hat{b}_{\eta}\left(\chi_{h}^{n}\right)-\hat{b}_{\eta}\left(\chi_{h}^{n-1}\right)}{\tau}, q_{h}^{n}\right\rangle \leq C_{\zeta}^{(5)}\left(\sum_{n=1}^{N} \tau\left\|q_{h}^{n}\right\|_{1, \mathcal{T}}^{2}\right)^{1 / 2} .
$$

Exploiting the stability of discrete gradients, $c f$., Lemma B.3, and a (continuous) Poincaré inequality (combined introducing $C_{\Omega, \mathrm{P}}$ ), the triangle inequality and the Cauchy-Schwarz inequality, we obtain

$$
\begin{aligned}
\sum_{n=1}^{N} \tau\left\|q_{h}^{n}\right\|_{1, \mathcal{T}}^{2} & \leq C_{\Omega, \mathrm{P}} \sum_{n=1}^{N} \tau\left\|\nabla q^{n}\right\|_{L^{2}(\Omega)}^{2}=C_{\Omega, \mathrm{P}} \sum_{n=1}^{N} \tau\left\|\tau^{-1} \int_{t_{n-1}}^{t_{n}} \nabla q \mathrm{~d} t\right\|_{L^{2}(\Omega)}^{2} \\
& \leq C_{\Omega, \mathrm{P}} \sum_{n=1}^{N} \tau^{-1}\left(\int_{t_{n-1}}^{t_{n}}\|\nabla q\| \mathrm{d} t\right)^{2} \leq C_{\Omega, \mathrm{P}} \sum_{n=1}^{N} \int_{t_{n-1}}^{t_{n}}\|\nabla q\|_{L^{2}(\Omega)}^{2} \mathrm{~d} t \\
& =C_{\Omega, \mathrm{P}}\|q\|_{L^{2}\left(0, T ; H_{0}^{1}(\Omega)\right)}^{2},
\end{aligned}
$$

which concludes the proof.

\subsection{Relative (weak) compactness for the limit $h, \tau \rightarrow 0$}

We utilize the stability results from the previous section to conclude relative compactness. We deduce limits for the interpolants which eventually converge towards a weak solution of the doubly regularized unsaturated poroelasticity model, i.e., it fulfills $(\mathrm{W} 1)_{\zeta \eta}-(\mathrm{W} 4)_{\zeta \eta}$.

Lemma 6.10 (Convergence of the mechanical displacement). There exist subsequences of $\left\{\overline{\boldsymbol{u}}_{h \tau}\right\}_{h, \tau}$ and $\left\{\hat{\boldsymbol{u}}_{h \tau}\right\}_{h, \tau}$, denoted by the same subscript, and $\boldsymbol{u}_{\zeta \eta} \in L^{\infty}(0, T ; \boldsymbol{V})$ with $\partial_{t} \boldsymbol{u}_{\zeta \eta} \in L^{2}(0, T ; \boldsymbol{V})$ such that for $h, \tau \rightarrow 0$

$$
\begin{aligned}
\overline{\boldsymbol{u}}_{h \tau} & \rightarrow \boldsymbol{u}_{\zeta \eta} & & \text { weakly in } L^{\infty}(0, T ; \boldsymbol{V}), \\
\overline{\boldsymbol{u}}_{h \tau} & \rightarrow \boldsymbol{u}_{\zeta \eta} & & \text { strongly in } L^{2}\left(Q_{T}\right), \\
\hat{\boldsymbol{u}}_{h \tau} & \rightarrow \boldsymbol{u}_{\zeta \eta} & & \text { weakly in } L^{2}(0, T ; \boldsymbol{V}), \\
\partial_{t} \hat{\boldsymbol{u}}_{h \tau} & \rightarrow \partial_{t} \boldsymbol{u}_{\zeta \eta} & & \text { weakly in } L^{2}(0, T ; \boldsymbol{V}) .
\end{aligned}
$$


Proof. The assertion essentially follows from the stability result in Lemma 6.6. In particular, equation (6.8a) follows together with the Eberlein-Šmulian theorem, $c f$. , Lemma B.8. For (6.8b), we employ a relaxed AubinLions-Simon type compactness result for Bochner spaces, $c f$., Lemma B.9. Furthermore, by the Eberlein-Šmulian theorem, there exists a $\hat{\boldsymbol{u}} \in L^{2}(0, T, \boldsymbol{V})$ such that up to a subsequence

$$
\begin{array}{cc}
\hat{\boldsymbol{u}}_{h \tau} \rightarrow \hat{\boldsymbol{u}} & \text { weakly in } L^{2}(0, T ; \boldsymbol{V}), \\
\partial_{t} \hat{\boldsymbol{u}}_{h \tau} \rightarrow \partial_{t} \hat{\boldsymbol{u}} & \text { weakly in } L^{2}(0, T ; \boldsymbol{V}) .
\end{array}
$$

We can identify $\hat{\boldsymbol{u}}=\boldsymbol{u}_{\zeta \eta}$ as follows. Employing the triangle inequality and Lemma 6.6, yields

$$
\begin{aligned}
\left\|\hat{\boldsymbol{u}}_{h \tau}-\boldsymbol{u}_{\zeta \eta}\right\|_{L^{2}\left(Q_{T}\right)} & \leq\left\|\hat{\boldsymbol{u}}_{h \tau}-\overline{\boldsymbol{u}}_{h \tau}\right\|_{L^{2}\left(Q_{T}\right)}+\left\|\overline{\boldsymbol{u}}_{h \tau}-\boldsymbol{u}_{\zeta \eta}\right\|_{L^{2}\left(Q_{T}\right)} \\
& \leq C^{(1)} \tau+\left\|\overline{\boldsymbol{u}}_{h \tau}-\boldsymbol{u}_{\zeta \eta}\right\|_{L^{2}\left(Q_{T}\right)},
\end{aligned}
$$

which converges to zero for $h, \tau \rightarrow 0$. This proves (6.8c) and (6.8d).

In order to discuss the limit of the pressure, we utilize techniques employed in the finite volume literature [19, 34]. For this, we define a piecewise constant discrete gradient of $\bar{\chi}_{h \tau}$ utilizing the dual grid $\mathcal{T}^{\star}, c f$. , Definition 5.2 ,

$$
(\bar{\nabla} \chi)_{h \tau}:=\left\{\begin{array}{lll}
d \frac{\chi_{\left.h\right|_{L}}^{n}-\chi_{\left.h\right|_{K}}^{n}}{d_{K \mid L}} \boldsymbol{n}_{K \mid L}, & (x, t) \in P_{K \mid L} \times\left(t_{n-1}, t_{n}\right], & K \in \mathcal{T}, L \in \mathcal{N}(K), \\
d \frac{\chi_{\left.h\right|_{K}}^{n} n_{\sigma, K},}{d_{\sigma, K}}, & (x, t) \in P_{\sigma} \times\left(t_{n-1}, t_{n}\right], & \sigma \in \mathcal{E}_{\text {ext }} \cap \mathcal{E}_{K},
\end{array}\right.
$$

where $\boldsymbol{n}_{K \mid L}$ denotes the outward normal on $K \mid L \in \mathcal{E}$, pointing towards $L$; and $\boldsymbol{n}_{\sigma, K}$ denotes the outward normal on $\sigma \in \mathcal{E}_{\text {ext }} \cap \mathcal{E}_{K}$, pointing towards $K$.

Lemma 6.11 (Convergence of the Kirchhoff pressure). There exists a subsequence of $\left\{\bar{\chi}_{h \tau}\right\}_{h, \tau}$, denoted by the same subscript, and $\chi_{\zeta \eta} \in H^{1}(0, T ; Q)$ such that

$$
\begin{aligned}
\bar{\chi}_{h \tau} & \rightarrow \chi_{\zeta \eta} & & \text { strongly in } L^{2}\left(Q_{T}\right), \\
(\bar{\nabla} \chi)_{h \tau} & \rightarrow \nabla \chi_{\zeta \eta} & & \text { weakly in } L^{2}\left(Q_{T}\right), \\
\partial_{t} \hat{\chi}_{h \tau} & \rightarrow \partial_{t} \chi_{\zeta \eta} & & \text { weakly in } L^{2}\left(Q_{T}\right) .
\end{aligned}
$$

Proof. Let $\hat{h} \in \mathbb{R}^{d}$ and $\Omega_{\hat{h}}:=\{x \in \Omega \mid x+\hat{h} \in \Omega\}$. Using a translation property for piecewise constant functions, $c f .$, e.g., Lemma 4 in [19], we obtain the following uniform translation property in space for $\bar{\chi}_{h \tau}$

$$
\begin{aligned}
\int_{0}^{T} \int_{\Omega_{\hat{h}}}\left\|\bar{\chi}_{h \tau}(x+\hat{h})-\bar{\chi}_{h \tau}(x)\right\|_{L^{2}(\Omega)}^{2} \mathrm{~d} x \mathrm{~d} t & =\sum_{n=1}^{N} \tau \int_{\Omega_{\hat{h}}}\left\|\chi_{h}^{n}(x+\hat{h})-\chi_{h}^{n}(x)\right\|_{L^{2}(\Omega)}^{2} \mathrm{~d} x \\
& \leq C|\hat{h}|(|\hat{h}|+|\Omega|) \sum_{n=1}^{N} \tau\left\|\chi_{h}^{n}\right\|_{1, \mathcal{T}}^{2}
\end{aligned}
$$

for some constant $C>0$. Consequently, together with Lemma $6.7, \bar{\chi}_{h \tau}$ satisfies a translation property in space and time wrt. $L^{2}\left(Q_{T}\right)$. We conclude by the Riesz-Frechet-Kolmogorov compactness criterion, $c f$., Lemma B.10, that there exists a $\chi_{\zeta \eta} \in L^{2}\left(Q_{T}\right)$ satisfying $(6.9 \mathrm{a})$.

For the proof of (6.9b), we refer to Proposition 8.1 in [34], which essentially proves the same assertion under the same assumption on uniform stability for $\sum_{n=1}^{N} \tau\left\|\chi_{h}^{n}\right\|_{1, \mathcal{T}}^{2}$, here following from Lemma 6.7.

The proof of $(6.9 \mathrm{c})$ is standard and follows mainly from the stability results in Lemma 6.7 and the EberleinŠmulian theorem, $c f$., Lemma B.8. This concludes the proof. 
The main purpose of the double regularization has been the aim to get control over the non-linear coupling terms, and eventually establish convergence.

Lemma 6.12 (Convergence of the coupling terms). There exists a subsequence of $\left\{\bar{\chi}_{h \tau}\right\}_{h, \tau}$, denoted by the same subscript, such that

$$
\begin{array}{rlrl}
\hat{p}_{\text {pore }}\left(\bar{\chi}_{h \tau}\right) & \rightarrow \hat{p}_{\text {pore }}\left(\chi_{\zeta \eta}\right) & \text { weakly in } L^{2}\left(Q_{T}\right), \\
\hat{s}_{\mathrm{w}}\left(\bar{\chi}_{h \tau}\right) \partial_{t} \boldsymbol{\nabla} \cdot \hat{\boldsymbol{u}}_{h \tau} & \rightarrow \hat{s}_{\mathrm{w}}\left(\chi_{\zeta \eta}\right) \partial_{t} \boldsymbol{\nabla} \cdot \boldsymbol{u}_{\zeta \eta} & & \text { weakly in } L^{2}\left(Q_{T}\right) .
\end{array}
$$

Proof. By the Eberlein-Šmulian theorem, cf., Lemmas B.8 and 6.8, there exists a subsequence of $\left\{\bar{\chi}_{h \tau}\right\}_{h, \tau}$, denoted by the same index, and a $\hat{p} \in L^{2}\left(Q_{T}\right)$ such that

$$
\hat{p}_{\text {pore }}\left(\bar{\chi}_{h \tau}\right) \rightarrow \hat{p} \quad \text { weakly in } L^{2}\left(Q_{T}\right) .
$$

The identification of $\hat{p}=\hat{p}_{\text {pore }}\left(\chi_{\zeta \eta}\right)$ follows from the continuity of $\hat{p}_{\text {pore }}, c f .,(\mathrm{A} 3)$, and the fact that $\bar{\chi}_{h \tau} \rightarrow \chi_{\zeta \eta}$ a.e. in $Q_{T}$ for a subsequence, denoted by the same index, $c f$., Lemma 6.11. This concludes (6.10a).

The convergence property $(6.10 \mathrm{~b})$ follows from the convergence properties of the single contributions. Let $q \in L^{2}\left(Q_{T}\right)$; it holds that $\hat{s}_{\mathrm{w}}\left(\bar{\chi}_{h \tau}\right) q \rightarrow \hat{s}_{\mathrm{w}}\left(\chi_{\zeta \eta}\right) q$ in $L^{2}\left(Q_{T}\right)$ (up to a subsequence). Indeed, by Lemma 6.11 , we have $\bar{\chi}_{h \tau} \rightarrow \chi_{\zeta \eta}$ a.e. in $Q_{T}$ (up to a subsequence); due to (A2), it holds that $\hat{s}_{\mathrm{W}}\left(\bar{\chi}_{h \tau}\right) q \rightarrow \hat{s}_{\mathrm{W}}\left(\chi_{\zeta \eta}\right) q$ a.e. in $Q_{T}$ and $\left|\hat{s}_{\mathrm{w}}\left(\bar{\chi}_{h \tau}\right) q\right| \leq|q|$ a.e. in $Q_{T}$; hence, by the dominated convergence theorem $\hat{s}_{\mathrm{w}}\left(\bar{\chi}_{h \tau}\right) q \rightarrow \hat{s}_{\mathrm{w}}\left(\chi_{\zeta \eta}\right) q$ in $L^{2}\left(Q_{T}\right)$. In particular, it holds that $\hat{s}_{\mathrm{w}}\left(\chi_{\zeta \eta}\right) q \in L^{2}(\Omega)$. Moreover from Lemma 6.10 , we have $\partial_{t} \boldsymbol{\nabla} \cdot \hat{\boldsymbol{u}}_{h \tau} \rightarrow \partial_{t} \boldsymbol{\nabla} \cdot \boldsymbol{u}_{\zeta \eta}$ in $L^{2}\left(Q_{T}\right)$. The product of weak and strong convergence results in

$$
\left\langle\partial_{t} \boldsymbol{\nabla} \cdot \hat{\boldsymbol{u}}_{h \tau}, \hat{s}_{\mathrm{w}}\left(\bar{\chi}_{h \tau}\right) q\right\rangle \rightarrow\left\langle\partial_{t} \boldsymbol{\nabla} \cdot \boldsymbol{u}_{\zeta \eta}, \hat{s}_{\mathrm{w}}\left(\chi_{\zeta \eta}\right) q\right\rangle, \quad h, \tau \rightarrow 0 .
$$

This proves (6.10b).

Lemma 6.13 (Initial conditions for the fluid flow). Let $\bar{\lambda}_{h \tau}$ as in (6.7). There exists a subsequence, denoted by the same index, such that

$$
\bar{\lambda}_{h \tau} \rightarrow \partial_{t} \hat{b}_{\eta}\left(\chi_{\zeta \eta}\right) \quad \text { weakly in } L^{2}\left(0, T ; Q^{\star}\right),
$$

where $\partial_{t} \hat{b}_{\eta}\left(\chi_{\zeta \eta}\right) \in L^{2}\left(0, T ; Q^{\star}\right)$ is understood in the sense of $(\mathrm{W} 2)_{\zeta \eta}$.

Proof. The stability result in Lemma 6.9 implies by the Eberlein-Šmulian theorem, $c f$. ., Lemma B.8, that there exist a subsequence of $\left\{\bar{\lambda}_{h \tau}\right\}_{h \tau}$, denoted by the same index, and a $\hat{b}_{t} \in L^{2}\left(0, T ; Q^{\star}\right)$ such that

$$
\bar{\lambda}_{h \tau} \rightarrow \hat{b}_{t} \quad \text { weakly in } L^{2}\left(0, T ; Q^{\star}\right) .
$$

It remains to show that $\hat{b}_{t}=\partial_{t} \hat{b}_{\eta}\left(\chi_{\zeta \eta}\right)$ in the sense of $(\mathrm{W} 2)_{\zeta \eta}$. We do this in three steps.

First, by Lemma 6.11 , there exists a subsequence of $\left\{\bar{\chi}_{h \tau}\right\}_{h \tau}$, denoted by the same index, such that $\bar{\chi}_{h \tau} \rightarrow \chi_{\zeta \eta}$ a.e. in $Q_{T}$. Since $\hat{b}_{\eta}$ is continuous, $c f .,(\mathrm{A} 1)$ and (4.1), it also holds $\hat{b}_{\eta}\left(\bar{\chi}_{h \tau}\right) \rightarrow \hat{b}_{\eta}\left(\chi_{\zeta \eta}\right)$ a.e. in $Q_{T}$. In addition, based on the definition of $\hat{B}_{\eta}, c f .,(4.2)$, and its properties, $c f$. , Lemma B.12, it holds that

$$
\left|\hat{b}_{\eta}\left(\bar{\chi}_{h \tau}\right)\right| \leq \delta \hat{B}_{\eta}\left(\bar{\chi}_{h \tau}\right)+\sup _{|y| \leq \delta^{-1}}\left|\hat{b}_{\eta}(y)\right|, \quad \text { a.e. in } Q_{T}
$$

for all $\delta>0$. Since $\hat{B}_{\eta}\left(\bar{\chi}_{h \tau}\right)$ is uniformly bounded in $L^{\infty}\left(0, T ; L^{1}(\Omega)\right)$ by Lemma 6.8 , we conclude

$$
\hat{b}_{\eta}\left(\bar{\chi}_{h \tau}\right) \rightarrow \hat{b}_{\eta}\left(\chi_{\zeta \eta}\right) \quad \text { strongly in } L^{1}\left(0, T ; L^{1}(\Omega)\right)
$$

by the dominated convergence theorem. By the properties of $\hat{B}_{\eta}$, it actually follows $\hat{b}_{\eta} \in L^{\infty}\left(0, T ; L^{1}(\Omega)\right)$. 
Second, a similar argument based on the strong convergence of $\left\{\chi_{h}^{0}\right\}_{h}$ in $L^{2}(\Omega), c f$., (5.2), the continuity of $\hat{b}_{\eta}$, the regularity assumption on $\hat{B}_{\eta}\left(\chi_{0}\right), c f .,(\mathrm{A} 8)_{\eta}$, and the properties of $\hat{B}_{\eta}$, cf., Lemma B.12, leads to

$$
\hat{b}_{\eta}\left(\chi_{h}^{0}\right) \rightarrow \hat{b}_{\eta}\left(\chi_{0}\right) \quad \text { strongly in } L^{1}(\Omega) .
$$

Third, let $q \in L^{2}(0, T ; Q) \cap W^{1,1}\left(0, T ; L^{\infty}(\Omega)\right)$ with $q(T)=0$. By definition of $\bar{\lambda}_{h \tau}$, after applying summation by parts, $c f$. , Lemma B.6, we obtain

$$
\begin{aligned}
\int_{0}^{T}\left\langle\bar{\lambda}_{h \tau}, q\right\rangle \mathrm{d} t= & \sum_{n=1}^{N}\left\langle\hat{b}_{\eta}\left(\chi_{h}^{n}\right)-\hat{b}_{\eta}\left(\chi_{h}^{n-1}\right), \tau^{-1} \int_{t_{n-1}}^{t_{n}} q \mathrm{~d} t\right\rangle \\
= & \left\langle\hat{b}_{\eta}\left(\chi_{h}^{N}\right)-\hat{b}_{\eta}\left(\chi_{h}^{0}\right), \tau^{-1} \int_{T-\tau}^{T} q \mathrm{~d} t\right\rangle \\
& +\int_{0}^{T-\tau}\left\langle\hat{b}_{\eta}\left(\chi_{h}^{0}\right)-\hat{b}_{\eta}\left(\bar{\chi}_{h \tau}\right), \partial_{t} \hat{q}_{\tau}-\partial_{t} q\right\rangle \mathrm{d} t \\
& +\int_{0}^{T-\tau}\left\langle\hat{b}_{\eta}\left(\chi_{h}^{0}\right)-\hat{b}_{\eta}\left(\bar{\chi}_{h \tau}\right), \partial_{t} q\right\rangle \mathrm{d} t \\
= & : T_{1}+T_{2}+T_{3} .
\end{aligned}
$$

In $T_{2}$, we have used the piecewise linear function $\hat{q}_{\tau}$, defined as

$$
\hat{q}_{\tau}(t):=\tau^{-1} \int_{t_{n}-\tau}^{t_{n}} q \mathrm{~d} t+\frac{t-\left(t_{n}-\tau\right)}{\tau}\left(\tau^{-1} \int_{t_{n}}^{t_{n}+\tau} q \mathrm{~d} t-\tau^{-1} \int_{t_{n}-\tau}^{t_{n}} q \mathrm{~d} t\right) .
$$

with $t \in\left(t_{n-1}, t_{n}\right]$ and $n \in\{1, \ldots, N-1\}$. Due to the uniform boundedness of $\left\|\hat{b}_{\eta}\left(\bar{\chi}_{h \tau}\right)\right\|_{L^{\infty}\left(0, T ; L^{1}(\Omega)\right)}$ and $\left\|\hat{b}_{\eta}\left(\chi_{h}^{0}\right)\right\|_{L^{1}(\Omega)}$ and since $\tau^{-1} \int_{T-\tau}^{T} q \mathrm{~d} t \rightarrow q(T)=0$ and $\partial_{t} \hat{q}_{\tau} \rightarrow \partial_{t} q$ in $L^{1}\left(0, T ; L^{\infty}(\Omega)\right)$ for $h, \tau \rightarrow 0$, it follows that $T_{1} \rightarrow 0$ and $T_{2} \rightarrow 0$ for $h, \tau \rightarrow 0$. Finally, the convergence in (6.12) and (6.13) result in

$$
T_{3} \rightarrow \int_{0}^{T}\left\langle\hat{b}_{\eta}\left(\chi_{0}\right)-\hat{b}_{\eta}\left(\chi_{\zeta \eta}\right), \partial_{t} q\right\rangle \mathrm{d} t, \quad \text { for } h, \tau \rightarrow 0,
$$

by the dominated convergence theorem. This concludes the proof.

Lemma 6.14 (Initial conditions for the mechanical displacement). The limit $\boldsymbol{u}_{\zeta \eta} \in H^{1}(0, T ; \boldsymbol{V})$ from Lemma 6.10 satisfies $(\mathrm{W} 3)_{\zeta \eta}$.

Proof. Let $\boldsymbol{v} \in H^{1}(0, T ; \boldsymbol{V})$ with $\boldsymbol{v}(T)=\mathbf{0}$. We obtain, using the same calculations as in the proof of Lemma 6.13,

$$
\int_{0}^{T} a\left(\partial_{t} \hat{\boldsymbol{u}}_{h \tau}, \boldsymbol{v}\right) \mathrm{d} t=a\left(\boldsymbol{u}_{h}^{N}-\boldsymbol{u}_{h}^{0}, \tau^{-1} \int_{T-\tau}^{T} \boldsymbol{v} \mathrm{d} t\right)+\int_{0}^{T-\tau} a\left(\boldsymbol{u}_{h}^{0}-\overline{\boldsymbol{u}}_{h \tau}, \partial_{t} \hat{\boldsymbol{v}}_{\tau}\right),
$$

with the piecewise linear function defined on $t \in\left(t_{n-1}, t_{n}\right], n \in\{1, \ldots, N-1\}$ by

$$
\hat{\boldsymbol{v}}_{\tau}(t)=\tau^{-1} \int_{t_{n-2}}^{t_{n-1}} \boldsymbol{v} \mathrm{d} t+\frac{t-t_{n-1}}{\tau}\left(\tau^{-1} \int_{t_{n-1}}^{t_{n}} \boldsymbol{v} \mathrm{d} t-\tau^{-1} \int_{t_{n-2}}^{t_{n-1}} \boldsymbol{v} \mathrm{d} t\right) .
$$

Based on the stability and convergence results for $\left\{\overline{\boldsymbol{u}}_{h \tau}\right\}_{h \tau}$ and $\left\{\boldsymbol{u}_{h}^{0}\right\}_{h}, c f .$, (5.4), we get (for a subsequence, denoted by the same index)

$$
\int_{0}^{T} a\left(\partial_{t} \boldsymbol{u}_{\zeta \eta}, \boldsymbol{v}\right) \mathrm{d} t=-\int_{0}^{T} a\left(\boldsymbol{u}_{\zeta \eta}-\boldsymbol{u}_{0}, \partial_{t} \boldsymbol{v}\right)
$$

for $h, \tau \rightarrow 0$ and thereby $(\mathrm{W} 3)_{\zeta \eta}$. 


\subsection{Identifying a weak solution for $h, \tau \rightarrow 0$}

Finally, we show the limit $\left(\boldsymbol{u}_{\zeta \eta}, \chi_{\zeta \eta}\right)$, introduced above, is a weak solution of the doubly regularized unsaturated poroelasticity model, $c f$. ., Definition 4.1.

Lemma 6.15 (Limit satisfies $\left.(\mathrm{W} 1)_{\zeta \eta}-(\mathrm{W} 4)_{\zeta \eta}\right)$. The limit $\left(\boldsymbol{u}_{\zeta \eta}, \chi_{\zeta \eta}\right)$ introduced in the previous section is a weak solution to the doubly regularized unsaturated poroelasticity model, cf., Definition 4.1.

Proof. The limit $\left(\boldsymbol{u}_{\zeta \eta}, \chi_{\zeta \eta}\right)$ satisfies $(\mathrm{W} 1)_{\zeta \eta}-(\mathrm{W} 3)_{\zeta \eta}$ by Lemmas $6.10,6.11,6.12$, and 6.14. It remains to show $(\mathrm{W} 4)_{\zeta \eta}$, i.e., that $\left(\boldsymbol{u}_{\zeta \eta}, \chi_{\zeta \eta}\right)$ satisfies the balance equations (4.4). We first consider sufficiently smooth test functions and then use a density argument. Let $(\boldsymbol{v}, q) \in L^{2}\left(0, T ; \boldsymbol{V} \cap C^{\infty}(\Omega)^{d}\right) \times L^{2}\left(0, T ; Q \cap C^{\infty}(\Omega)\right)$. For a given mesh $\mathcal{T}$, we define spatial projection and interpolation operators, respectively, by

$$
\begin{array}{lll}
\Pi_{\boldsymbol{V}_{h}}: \boldsymbol{V} \cap C^{\infty}(\Omega) \rightarrow \boldsymbol{V}_{h}, & \text { s.t. }\left\langle\Pi_{\boldsymbol{V}_{h}} \boldsymbol{v}, \boldsymbol{v}_{h}\right\rangle=\left\langle\boldsymbol{v}, \boldsymbol{v}_{h}\right\rangle & \text { for all } \boldsymbol{v}_{h} \in \boldsymbol{V}_{h}, \\
\mathcal{I}_{Q_{h}}: Q \cap C^{\infty}(\Omega) \rightarrow Q_{h}, & \text { s.t. } \mathcal{I}_{Q_{h}} q_{\left.\right|_{K}}=q\left(x_{K}\right) & \text { for all } K \in \mathcal{T} .
\end{array}
$$

Using those, we define piecewise-constant-in-time interpolants of $(\boldsymbol{v}, q)$

$$
\begin{gathered}
\overline{\boldsymbol{v}}_{h \tau}(t):=\boldsymbol{v}_{h}^{n}, \quad t \in\left(t_{n-1}, t_{n}\right], \quad \boldsymbol{v}_{h}^{n}:=\Pi_{\boldsymbol{V}_{h}} \boldsymbol{v}^{n}, \quad \boldsymbol{v}^{n}:=\tau^{-1} \int_{t_{n-1}}^{t_{n}} \boldsymbol{v} \mathrm{d} t, \\
\bar{q}_{h \tau}(t):=q_{h}^{n}, \quad t \in\left(t_{n-1}, t_{n}\right], \quad q_{h}^{n}:=\mathcal{I}_{Q_{h}} q^{n}, \quad q^{n}:=\tau^{-1} \int_{t_{n-1}}^{t_{n}} q \mathrm{~d} t .
\end{gathered}
$$

Similarly, let

$$
\begin{array}{ll}
\overline{\boldsymbol{f}}_{\text {ext }, \tau}(t):=\boldsymbol{f}_{\mathrm{ext}}^{n}, & t \in\left(t_{n-1}, t_{n}\right], \\
\bar{h}_{\text {ext }, \tau}(t):=h_{\mathrm{ext}}^{n}, & t \in\left(t_{n-1}, t_{n}\right] .
\end{array}
$$

Combining classical results, based on the assumed regularity (A7), for $h, \tau \rightarrow 0$ it holds that

$$
\begin{aligned}
\overline{\boldsymbol{v}}_{h \tau} & \rightarrow \boldsymbol{v} & & \text { strongly in } L^{2}(0, T ; \boldsymbol{V}), \\
\bar{q}_{h \tau} & \rightarrow q & & \text { strongly in } L^{2}(0, T ; Q), \\
\overline{\boldsymbol{f}}_{\text {ext }, \tau} & \rightarrow \boldsymbol{f}_{\text {ext }} & & \text { strongly in } L^{2}\left(0, T ; \boldsymbol{V}^{\star}\right), \\
\bar{h}_{\mathrm{ext}, \tau} & \rightarrow h_{\text {ext }} & & \text { strongly in } L^{2}\left(0, T ; Q^{\star}\right) .
\end{aligned}
$$

We choose $\boldsymbol{v}_{h}=\boldsymbol{v}_{h}^{n}$ and $q_{h}=q_{h}^{n}$ as test functions in (5.5), multiply both equations with $\tau$ and sum over all time steps 1 to $N$; we obtain

$$
\begin{array}{r}
\int_{0}^{T}\left[\zeta a\left(\partial_{t} \hat{\boldsymbol{u}}_{h \tau}, \overline{\boldsymbol{v}}_{h \tau}\right)+a\left(\overline{\boldsymbol{u}}_{h \tau}, \overline{\boldsymbol{v}}_{h \tau}\right)-\alpha\left\langle\hat{p}_{\text {pore }}\left(\bar{\chi}_{h \tau}\right), \boldsymbol{\nabla} \cdot \overline{\boldsymbol{v}}_{h \tau}\right\rangle\right] \mathrm{d} t=\int_{0}^{T}\left\langle\overline{\boldsymbol{f}}_{\mathrm{ext}, \tau}, \overline{\boldsymbol{v}}_{h \tau}\right\rangle \mathrm{d} t \\
\int_{0}^{T}\left[\left\langle\bar{\lambda}_{h \tau}, \bar{q}_{h \tau}\right\rangle+\alpha\left\langle\hat{s}_{\mathrm{w}}\left(\bar{\chi}_{h \tau}\right) \partial_{t} \boldsymbol{\nabla} \cdot \hat{\boldsymbol{u}}_{h \tau}, \bar{q}_{h \tau}\right\rangle+\left\langle\boldsymbol{\nabla}_{h} \bar{\chi}_{h \tau}, \nabla_{h} \bar{q}_{h \tau}\right\rangle_{\kappa_{\mathrm{abs}}}\right] \mathrm{d} t=\int_{0}^{T}\left\langle\bar{h}_{\mathrm{ext}, \tau}, \bar{q}_{h \tau}\right\rangle \mathrm{d} t .
\end{array}
$$

For most terms we can apply the fact that the product of weakly and strongly convergent sequences converge to the product of their limits. The only term needing special attention is the diffusion term in the flow equation. For this, we follow a similar argument as in the proof of Theorem 3.4 in [34] - here for a heterogeneous permeability field. The technical calculations are the same. Hence, we only summarize the idea.

We exploit the definition of the piecewise constant gradient $(\overline{\nabla \chi})_{h \tau}$. Similarly, we define a piecewise constant gradient of the smooth test function and the permeability field

$$
(\bar{\nabla} q)_{h \tau}(x, t):=\nabla q^{n}\left(x_{\sigma}\right), \quad(x, t) \in P_{\sigma} \times\left(t_{n-1}, t_{n}\right], \quad \sigma \in \mathcal{E},
$$




$$
\left\{\kappa_{\mathrm{abs}}\right\}_{\mathcal{T}}(x):=\left\{\kappa_{\mathrm{abs}}\right\}_{\sigma}, \quad x \in P_{\sigma}, \quad \sigma \in \mathcal{E},
$$

where $x_{\sigma} \in P_{\sigma}$ is chosen by the mean value theorem satisfying (here only for an internal edge) $\frac{1}{d_{K \mid L}}\left(q^{n}\left(x_{K}\right)-q^{n}\left(x_{L}\right)\right)=\boldsymbol{\nabla} q^{n}\left(x_{K \mid L}\right) \cdot \boldsymbol{n}_{L \mid K}$, i.e., connecting the two-point flux approximation with the actual gradient of $q$. A calculation, just as in [34], yields

$$
\int_{0}^{T}\left\langle\nabla_{h} \bar{\chi}_{h \tau}, \nabla_{h} \bar{q}_{h \tau}\right\rangle_{\kappa_{\mathrm{abs}}} \mathrm{d} t=\int_{0}^{T} \int_{\Omega}\left\{\kappa_{\mathrm{abs}}\right\}_{\mathcal{T}}(\bar{\nabla} \chi)_{h \tau} \cdot(\bar{\nabla} q)_{h \tau} \mathrm{d} x \mathrm{~d} t .
$$

Due to sufficient regularity, it holds that $\left\{\kappa_{\text {abs }}\right\}_{\mathcal{T}} \rightarrow \kappa_{\text {abs }}$ strongly in $L^{\infty}\left(Q_{T}\right)$ and $(\bar{\nabla} q)_{h \tau} \rightarrow \nabla q$ strongly in $L^{2}\left(Q_{T}\right)$. Since by Lemma 6.11 it also holds that $(\bar{\nabla} \chi)_{h \tau} \rightarrow \nabla \chi_{\zeta \eta}$ weakly in $L^{2}\left(Q_{T}\right)$, we finally conclude that

$$
\int_{0}^{T}\left\langle\nabla_{h} \bar{\chi}_{h \tau}, \nabla_{h} \bar{q}_{h \tau}\right\rangle_{\kappa_{\mathrm{abs}}} \mathrm{d} t \rightarrow \int_{0}^{T} \int_{\Omega} \kappa_{\mathrm{abs}} \nabla \chi_{\zeta \eta} \cdot \nabla q \mathrm{~d} x \mathrm{~d} t, \quad h, \tau \rightarrow 0 .
$$

All in all, together with the convergence properties of the test functions $\overline{\boldsymbol{v}}_{h \tau}, \bar{q}_{h \tau}$, the source terms $\overline{\boldsymbol{f}}_{\text {ext, } \tau}$, $\bar{h}_{\text {ext }, \tau}$, and the interpolants for the fully discrete approximations (cf., Lems. 6.10-6.13), we conclude that (6.18) converges to (4.4), evaluated in $\left(\boldsymbol{u}_{\zeta \eta}, \chi_{\zeta \eta}\right)$ and tested with $(\boldsymbol{v}, q) \in L^{2}\left(0, T ; \boldsymbol{V} \cap C^{\infty}(\Omega)^{d}\right) \times L^{2}\left(0, T ; Q \cap C^{\infty}(\Omega)\right)$. Finally, a density argument yields the final result.

\section{Step 4: InCREASED REgularity in A NON-DEgenerate CASE}

In the following, further stability estimates for the fully-discrete problem are derived, allowing for showing that the limit $\left(\boldsymbol{u}_{\zeta \eta}, \chi_{\zeta \eta}\right)$ introduced in the previous section also satisfies (W5) $)_{\zeta \eta}$ and $(\mathrm{W} 6)_{\zeta \eta}$, i.e., we prove Lemma 4.5. For this, non-degeneracy assumptions are required. For compact presentation throughout the entire section, we assume (A0)-(A8) and (ND1)-(ND2) hold true, and we define $\boldsymbol{u}_{h}^{-1}:=\boldsymbol{u}_{h}^{0}$.

\subsection{Improved stability estimates for fully-discrete approximation}

Lemma 7.1 (Improved stability estimate for the structural velocity). There exists a constant $C_{\zeta \eta}^{(6)}>0$ (independent of $h, \tau)$, satisfying

$$
\begin{aligned}
& \zeta \sup _{n \in\{1, \ldots, N\}}\left\|\tau^{-1}\left(\boldsymbol{u}_{h}^{n}-\boldsymbol{u}_{h}^{n-1}\right)\right\|_{\boldsymbol{V}}^{2}+\sum_{n=1}^{N} \tau^{-1}\left\|\boldsymbol{u}_{h}^{n}-\boldsymbol{u}_{h}^{n-1}\right\|_{\boldsymbol{V}}^{2} \\
& \quad+\zeta \sum_{n=1}^{N}\left\|\tau^{-1}\left(\boldsymbol{u}_{h}^{n}-\boldsymbol{u}_{h}^{n-1}\right)-\tau^{-1}\left(\boldsymbol{u}_{h}^{n-1}-\boldsymbol{u}_{h}^{n-2}\right)\right\|_{\boldsymbol{V}}^{2} \\
& \quad+\sum_{n=1}^{N} \tau^{-1}\left\|\hat{p}_{\text {pore }}\left(\chi_{h}^{n}\right)-\hat{p}_{\text {pore }}\left(\chi_{h}^{n-1}\right)\right\|_{L^{2}(\Omega)}^{2} \\
& \leq C_{\zeta \eta}^{(6)}\left(\left\|\partial_{t} \boldsymbol{f}_{\text {ext }}\right\|_{L^{2}\left(Q_{T}\right)}^{2}, \frac{C_{\mathrm{ND}, 2}}{b_{\chi, \mathrm{m}}} C_{\zeta \eta}^{(2)}\right),
\end{aligned}
$$

where $C_{\zeta \eta}^{(2)}$ is the stability constant from Lemma 6.2, $C_{\mathrm{ND}, 2}$ corresponds to the non-degeneracy condition (ND2), and $b_{\chi, \mathrm{m}}$ corresponds to the growth condition $\left(\mathrm{A} 1^{\star}\right)$.

Proof. First we observe, that the compatibility condition for the initial conditions (5.3) is equivalent to the mechanics equation (5.5a) for $n=0$, since $\boldsymbol{u}_{h}^{0}-\boldsymbol{u}_{h}^{-1}=\mathbf{0}$. This allows for considering the difference of the mechanics equation (5.5a) at time steps $n$ and $n-1, n \geq 1$,

$$
\begin{gathered}
\zeta a\left(\tau^{-1}\left(\boldsymbol{u}_{h}^{n}-\boldsymbol{u}_{h}^{n-1}\right)-\tau^{-1}\left(\boldsymbol{u}_{h}^{n-1}-\boldsymbol{u}_{h}^{n-2}\right), \boldsymbol{v}_{h}\right)+a\left(\boldsymbol{u}_{h}^{n}-\boldsymbol{u}_{h}^{n-1}, \boldsymbol{v}_{h}\right) \\
-\alpha\left\langle\hat{p}_{\text {pore }}\left(\chi_{h}^{n}\right)-\hat{p}_{\text {pore }}\left(\chi_{h}^{n-1}\right), \boldsymbol{\nabla} \cdot \boldsymbol{v}_{h}\right\rangle=\left\langle\boldsymbol{f}_{\text {ext }}^{n}-\boldsymbol{f}_{\text {ext }}^{n-1}, \boldsymbol{v}_{h}\right\rangle
\end{gathered}
$$


for all $\boldsymbol{v}_{h} \in \boldsymbol{V}_{h}$. By testing with $\boldsymbol{v}_{h}=\tau^{-1}\left(\boldsymbol{u}_{h}^{n}-\boldsymbol{u}_{h}^{n-1}\right)$ and using the binomial identity (App. B.2), we obtain

$$
\begin{aligned}
& \frac{\zeta}{2}\left(\left\|\tau^{-1}\left(\boldsymbol{u}_{h}^{n}-\boldsymbol{u}_{h}^{n-1}\right)\right\|_{\boldsymbol{V}}^{2}-\left\|\tau^{-1}\left(\boldsymbol{u}_{h}^{n-1}-\boldsymbol{u}_{h}^{n-2}\right)\right\|_{\boldsymbol{V}}^{2}\right. \\
& \left.\quad+\left\|\tau^{-1}\left(\boldsymbol{u}_{h}^{n}-\boldsymbol{u}_{h}^{n-1}\right)-\tau^{-1}\left(\boldsymbol{u}_{h}^{n-1}-\boldsymbol{u}_{h}^{n-2}\right)\right\|_{\boldsymbol{V}}^{2}\right)+\tau^{-1}\left\|\boldsymbol{u}_{h}^{n}-\boldsymbol{u}_{h}^{n-1}\right\|_{\boldsymbol{V}}^{2} \\
& \quad=\tau^{-1}\left\langle\boldsymbol{f}_{\text {ext }}^{n}-\boldsymbol{f}_{\text {ext }}^{n-1}, \boldsymbol{u}_{h}^{n}-\boldsymbol{u}_{h}^{n-1}\right\rangle+\alpha \tau^{-1}\left\langle\hat{p}_{\text {pore }}\left(\chi_{h}^{n}\right)-\hat{p}_{\text {pore }}\left(\chi_{h}^{n-1}\right), \boldsymbol{\nabla} \cdot\left(\boldsymbol{u}_{h}^{n}-\boldsymbol{u}_{h}^{n-1}\right)\right\rangle .
\end{aligned}
$$

Summing over $n \in\{1, \ldots, N\}$, yields after applying the Cauchy-Schwarz inequality and Young's inequality for the right hand side terms

$$
\begin{aligned}
& \frac{\zeta}{2}\left\|\tau^{-1}\left(\boldsymbol{u}_{h}^{N}-\boldsymbol{u}_{h}^{N-1}\right)\right\|_{\boldsymbol{V}}^{2}+\frac{1}{2} \sum_{n=1}^{N} \tau^{-1}\left\|\boldsymbol{u}_{h}^{n}-\boldsymbol{u}_{h}^{n-1}\right\|_{\boldsymbol{V}}^{2} \\
& \quad+\frac{\zeta}{2} \sum_{n=1}^{N}\left\|\tau^{-1}\left(\boldsymbol{u}_{h}^{n}-\boldsymbol{u}_{h}^{n-1}\right)-\tau^{-1}\left(\boldsymbol{u}_{h}^{n-1}-\boldsymbol{u}_{h}^{n-2}\right)\right\|_{\boldsymbol{V}}^{2} \\
& \leq \sum_{n=1}^{N} \tau^{-1}\left\|\boldsymbol{f}_{\mathrm{ext}}^{n}-\boldsymbol{f}_{\mathrm{ext}}^{n-1}\right\|_{\boldsymbol{V}^{\star}}^{2}+\frac{\alpha^{2}}{K_{\mathrm{dr}}} \sum_{n=1}^{N} \tau^{-1}\left\|\hat{p}_{\text {pore }}\left(\chi_{h}^{n}\right)-\hat{p}_{\text {pore }}\left(\chi_{h}^{n-1}\right)\right\|_{L^{2}(\Omega)}^{2}
\end{aligned}
$$

Due to (ND2), $\hat{p}_{\text {pore }}=\hat{p}_{\text {pore }}(\chi)$ is Lipschitz continuous. Therefore, by Lemma 6.2 it holds that

$$
\sum_{n=1}^{N} \tau^{-1}\left\|\hat{p}_{\text {pore }}\left(\chi_{h}^{n}\right)-\hat{p}_{\text {pore }}\left(\chi_{h}^{n-1}\right)\right\|_{L^{2}(\Omega)}^{2} \leq C_{\mathrm{ND}, 2}^{2} \frac{C_{\zeta \eta}^{(2)}}{b_{\chi, \mathrm{m}}},
$$

which together with (7.1) concludes the proof.

Lemma 7.2 (Consequence for the structural acceleration). There exists a constant $C_{\zeta \eta}^{(7)}>0$ (independent of $h, \tau)$, such that

$$
\sum_{n=1}^{N} \tau\left\|\frac{\boldsymbol{u}_{h}^{n}-2 \boldsymbol{u}_{h}^{n-1}+\boldsymbol{u}_{h}^{n-2}}{\tau^{2}}\right\|_{\boldsymbol{V}}^{2} \leq C_{\zeta \eta}^{(7)}\left(\zeta^{-2} C_{\zeta \eta}^{(6)}\right)
$$

where $C_{\zeta \eta}^{(6)}$ is the stability constant from Lemma 7.1 .

Proof. Let $\left\{\boldsymbol{v}_{h}^{n}\right\}_{n} \subset \boldsymbol{V}_{h} \backslash\{\mathbf{0}\}$ be an arbitrary sequence of test functions. Consider the difference of (5.5a) at $n$ and $n-1, n \geq 1$, tested with $\boldsymbol{v}_{h}^{n}$; it holds that

$$
\begin{aligned}
\tau^{-1} \zeta a\left(\boldsymbol{u}_{h}^{n}-2 \boldsymbol{u}_{h}^{n-1}+\boldsymbol{u}_{h}^{n-2}, \boldsymbol{v}_{h}^{n}\right)= & \left\langle\boldsymbol{f}_{\text {ext }}^{n}-\boldsymbol{f}_{\text {ext }}^{n-1}, \boldsymbol{v}_{h}^{n}\right\rangle-a\left(\boldsymbol{u}_{h}^{n}-\boldsymbol{u}_{h}^{n-1}, \boldsymbol{v}_{h}^{n}\right) \\
& +\alpha\left\langle\hat{p}_{\text {pore }}\left(\chi_{h}^{n}\right)-\hat{p}_{\text {pore }}\left(\chi_{h}^{n-1}\right), \boldsymbol{\nabla} \cdot \boldsymbol{v}_{h}^{n}\right\rangle .
\end{aligned}
$$

Summing over $n \in\{1, \ldots, N\}$, applying the Cauchy-Schwarz inequality and Lemma 7.1, yields

$$
\sup _{\left\{\boldsymbol{v}_{h}^{n}\right\}_{n} \subset \boldsymbol{V}_{h} \backslash\{\mathbf{0}\}} \frac{\zeta \sum_{n=1}^{N} \tau^{-1} a\left(\boldsymbol{u}_{h}^{n}-2 \boldsymbol{u}_{h}^{n-1}+\boldsymbol{u}_{h}^{n-2}, \boldsymbol{v}_{h}^{n}\right)}{\left(\sum_{n=1}^{N} \tau\left\|\boldsymbol{v}_{h}^{n}\right\|_{\boldsymbol{V}}^{2}\right)^{1 / 2}} \leq 3 \sqrt{C_{\zeta \eta}^{(6)}} .
$$

As $\|\cdot\|_{\boldsymbol{V}}^{2}=a(\cdot, \cdot)$, we obtain equivalence of norms, which concludes the proof. 


\subsection{Improved stability estimates for interpolants in time}

We define piecewise linear interpolations of the discrete structural velocity and the pore pressure. For $t \in$ $\left(t_{n-1}, t_{n}\right], n \geq 1$, let

$$
\begin{aligned}
\hat{\boldsymbol{u}}_{t, h \tau}(t) & :=\frac{\boldsymbol{u}_{h}^{n-1}-\boldsymbol{u}_{h}^{n-2}}{\tau}+\frac{t-t_{n-1}}{\tau} \frac{\boldsymbol{u}_{h}^{n}-2 \boldsymbol{u}_{h}^{n-1}+\boldsymbol{u}_{h}^{n-2}}{\tau}, \\
\hat{p}_{\text {pore }, h \tau}(t) & :=\hat{p}_{\text {pore }}\left(\chi_{h}^{n-1}\right)+\frac{t-t_{n-1}}{\tau}\left(\hat{p}_{\text {pore }}\left(\chi_{h}^{n}\right)-\hat{p}_{\text {pore }}\left(\chi_{h}^{n-1}\right)\right) .
\end{aligned}
$$

Note that $\partial_{t} \hat{\boldsymbol{u}}_{h \tau}$ defines the piecewise constant analog of $\hat{\boldsymbol{u}}_{t, h \tau}$. Stability bounds are obtained as direct consequences of Lemmas 7.1 and 7.2.

Lemma 7.3 (Stability estimates for interpolations of the structural velocity). Let $\hat{\boldsymbol{u}}_{h \tau}$ and $\hat{\boldsymbol{u}}_{t, h \tau}$, as defined by (6.4) and (7.2a). For all $h, \tau>0$ and $\hat{\tau} \in(0, \tau)$, it holds that

$$
\begin{aligned}
\left\|\partial_{t} \hat{\boldsymbol{u}}_{h \tau}\right\|_{L^{2}(0, T ; \boldsymbol{V})}^{2} & \leq C_{\zeta \eta}^{(6)}, \\
\int_{0}^{T-\hat{\tau}}\left\|\partial_{t} \hat{\boldsymbol{u}}_{h \tau}(t+\hat{\tau})-\partial_{t} \hat{\boldsymbol{u}}_{h \tau}(t)\right\|_{L^{2}(\Omega)}^{2} \mathrm{~d} t & \leq C_{\Omega, \mathrm{PK}}^{2} C_{\zeta \eta}^{(6)} \hat{\tau} \\
\left\|\hat{\boldsymbol{u}}_{t, h \tau}\right\|_{L^{2}(0, T ; \boldsymbol{V})}^{2} & \leq 2 C_{\zeta \eta}^{(6)}, \\
\left\|\hat{\boldsymbol{u}}_{t, h \tau}-\partial_{t} \hat{\boldsymbol{u}}_{h \tau}\right\|_{L^{2}\left(Q_{T}\right)}^{2} & \leq \frac{C_{\Omega, \mathrm{PK}}^{2} C_{\zeta \eta}^{(7)}}{\zeta} \tau^{2}, \\
\left\|\partial_{t} \hat{\boldsymbol{u}}_{t, h \tau}\right\|_{L^{2}(0, T ; \boldsymbol{V})}^{2} & \leq \frac{C_{\zeta \eta}^{(7)}}{\zeta}
\end{aligned}
$$

where $C_{\zeta \eta}^{(6)}$ and $C_{\zeta \eta}^{(7)}$ are the stability constants from Lemmas 7.1 and $\% .2$, respectively, and $C_{\Omega, \mathrm{PK}}$ is the product of the Poincaré and the Korn constants.

Proof. By construction, it holds that

$$
\left\|\partial_{t} \hat{\boldsymbol{u}}_{h \tau}\right\|_{L^{2}(0, T ; \boldsymbol{V})}^{2}=\sum_{n=1}^{N} \tau^{-1}\left\|\boldsymbol{u}_{h}^{n}-\boldsymbol{u}_{h}^{n-1}\right\|_{\boldsymbol{V}}^{2}
$$

Hence, (7.3a) follows directly from Lemma 7.1. The time-translation property (7.3b) follows from the fact that $\partial_{t} \hat{\boldsymbol{u}}_{h \tau}$ is piecewise constant. Analogous to the proof of Lemma 6.6, one can show

$$
\int_{0}^{T-\tau}\left\|\partial_{t} \hat{\boldsymbol{u}}_{h \tau}(t+\tau)-\partial_{t} \hat{\boldsymbol{u}}_{h \tau}(t)\right\|_{L^{2}(\Omega)}^{2} \mathrm{~d} t=\hat{\tau} \sum_{n=1}^{N}\left\|\tau^{-1}\left(\boldsymbol{u}_{h}^{n}-\boldsymbol{u}_{h}^{n-1}\right)-\tau^{-1}\left(\boldsymbol{u}_{h}^{n-1}-\boldsymbol{u}_{h}^{n-2}\right)\right\|_{L^{2}(\Omega)}^{2} .
$$

Finally, after using a Poincaré inequality and Korn's inequality, (7.3b) follows from Lemma 7.1.

In order to show (7.3c), we expand the integral over the time interval. By definition of $\hat{\boldsymbol{u}}_{t, h \tau}$, it holds that

$$
\begin{aligned}
& \left\|\hat{\boldsymbol{u}}_{t, h \tau}\right\|_{L^{2}(0, T ; \boldsymbol{V})}^{2} \\
& \quad=\sum_{n=1}^{N} \tau^{-2} \int_{t_{n-1}}^{t_{n}}\left\|\left(\boldsymbol{u}_{h}^{n-1}-\boldsymbol{u}_{h}^{n-2}\right)+\frac{t-t_{n-1}}{\tau}\left(\boldsymbol{u}_{h}^{n}-2 \boldsymbol{u}_{h}^{n-1}+\boldsymbol{u}_{h}^{n-2}\right)\right\|_{\boldsymbol{V}}^{2} \mathrm{~d} t \\
& \quad \leq 2 \sum_{n=2}^{N} \tau^{-2} \int_{t_{n-1}}^{t_{n}}\left(\frac{t-t_{n}}{\tau}\right)^{2}\left\|\boldsymbol{u}_{h}^{n-1}-\boldsymbol{u}_{h}^{n-2}\right\|_{\boldsymbol{V}}^{2} \mathrm{~d} t+2 \sum_{n=2}^{N} \tau^{-2} \int_{t_{n-1}}^{t_{n}}\left(\frac{t-t_{n-1}}{\tau}\right)^{2}\left\|\boldsymbol{u}_{h}^{n}-\boldsymbol{u}_{h}^{n-1}\right\|_{\boldsymbol{V}}^{2} \mathrm{~d} t
\end{aligned}
$$




$$
\leq \frac{4}{3} \sum_{n=1}^{N} \tau^{-1}\left\|\boldsymbol{u}_{h}^{n}-\boldsymbol{u}_{h}^{n-1}\right\|_{V^{2}}^{2}
$$

Hence, equation (7.3c) follows by Lemma 7.1. In order to show (7.3d), we again expand the integral over the time interval. Employing the definitions of $\hat{\boldsymbol{u}}_{t, h \tau}$ and $\hat{\boldsymbol{u}}_{h \tau}$, a simple calculation yields

$$
\left\|\hat{\boldsymbol{u}}_{t, h \tau}-\partial_{t} \hat{\boldsymbol{u}}_{h \tau}\right\|_{L^{2}\left(Q_{T}\right)}^{2}=\frac{1}{3} \sum_{n=1}^{N} \tau^{-1}\left\|\boldsymbol{u}_{h}^{n}-2 \boldsymbol{u}_{h}^{n-1}+\boldsymbol{u}_{h}^{n-2}\right\|_{L^{2}(\Omega)}^{2} .
$$

Hence, after employing a Poincaré inequality and Korn's inequality, equation (7.3d) follows from Lemma 7.2. Finally, equation (7.3e) follows directly from Lemma 7.2, since

$$
\left\|\partial_{t} \hat{\boldsymbol{u}}_{t, h \tau}\right\|_{L^{2}(0, T ; \boldsymbol{V})}^{2}=\sum_{n=1}^{N} \tau\left\|\frac{\boldsymbol{u}_{h}^{n}-2 \boldsymbol{u}_{h}^{n-1}+\boldsymbol{u}_{h}^{n-2}}{\tau^{2}}\right\|_{\boldsymbol{V}}^{2} .
$$

Lemma 7.4 (Stability result for the interpolation of the pore pressure). For $\hat{p}_{\text {pore }, h \tau}$ defined in (7.2b). It holds that

$$
\begin{aligned}
\left\|\partial_{t} \hat{p}_{\text {pore }, h \tau}\right\|_{L^{2}\left(Q_{T}\right)}^{2} & \leq C_{\zeta \eta}^{(6)}, \\
\left\|\hat{p}_{\text {pore }, h \tau}-\hat{p}_{\text {pore }}\left(\bar{\chi}_{h \tau}\right)\right\|_{L^{2}\left(Q_{T}\right)}^{2} & \leq C_{\zeta \eta}^{(6)} \tau^{2},
\end{aligned}
$$

where $C_{\zeta \eta}^{(6)}$ is the stability constant from Lemma \%.1.

Proof. By construction, it holds that

$$
\begin{aligned}
\left\|\partial_{t} \hat{p}_{\text {pore }, h \tau}\right\|_{L^{2}\left(Q_{T}\right)}^{2} & =\sum_{n=1}^{N} \tau^{-1}\left\|\hat{p}_{\text {pore }}\left(\chi_{h}^{n}\right)-\hat{p}_{\text {pore }}\left(\chi_{h}^{n-1}\right)\right\|_{L^{2}(\Omega)}^{2}, \quad \text { and } \\
\left\|\hat{p}_{\text {pore }, h \tau}-\hat{p}_{\text {pore }}\left(\bar{\chi}_{h \tau}\right)\right\|_{L^{2}\left(Q_{T}\right)}^{2} & =\sum_{n=1}^{N} \frac{\tau}{3}\left\|\hat{p}_{\text {pore }}\left(\chi_{h}^{n}\right)-\hat{p}_{\text {pore }}\left(\chi_{h}^{n-1}\right)\right\|_{L^{2}(\Omega)}^{2},
\end{aligned}
$$

where the second result follows by expanding time integration. Hence, the assertion follows directly from the stability result for the discrete time derivative of the pore pressure, $c f$. , Lemma 7.1.

\subsection{More relative (weak) compactness for $h, \tau \rightarrow 0$}

The previous stability results allow for analyzing the limit in relation to $\left(\boldsymbol{u}_{\zeta \eta}, \chi_{\zeta \eta}\right)$.

Lemma 7.5 (Convergence of the structural velocity and acceleration). There exist subsequences subsequences of $\left\{\hat{\boldsymbol{u}}_{h \tau}\right\}_{h, \tau}$ and $\left\{\hat{\boldsymbol{u}}_{t, h \tau}\right\}_{h, \tau}$, denoted by the same subscript, such that $\partial_{t} \boldsymbol{u}_{\zeta \eta}, \partial_{t t} \boldsymbol{u}_{\zeta \eta} \in L^{2}(0, T ; \boldsymbol{V})$ and

$$
\begin{aligned}
\partial_{t} \hat{\boldsymbol{u}}_{h \tau} & \rightarrow \partial_{t} \boldsymbol{u}_{\zeta \eta} & & \text { weakly in } L^{2}(0, T ; \boldsymbol{V}), \\
\partial_{t} \hat{\boldsymbol{u}}_{t, h \tau} & \rightarrow \partial_{t t} \boldsymbol{u}_{\zeta \eta} & & \text { weakly in } L^{2}(0, T ; \boldsymbol{V}) .
\end{aligned}
$$

Proof. The convergence result (7.4a) follows from the stability result (7.3a), the Eberlein-Šmulian theorem, $c f$., Lemma B.8, and the fact that $\hat{\boldsymbol{u}}_{h \tau} \rightarrow \boldsymbol{u}_{\zeta \eta}$ weakly in $L^{2}(0, T ; \boldsymbol{V}), c f$., Lemma 6.10 . Furthermore, due to the additional translation property (7.3b), a relaxed Aubin-Lions-Simon type compactness result for Bochner spaces, $c f$. ., Lemma B.9 yields the existence of a subsequence, denoted by the same index, satisfying

$$
\partial_{t} \hat{\boldsymbol{u}}_{h \tau} \rightarrow \partial_{t} \boldsymbol{u}_{\zeta \eta} \quad \text { strongly in } L^{2}\left(Q_{T}\right) .
$$


Using the stability result (7.3e), by the Eberlein-Šmulian theorem, $c f$. , Lemma B.8, there exists a subsequence, denoted by the same index, such that $\partial_{t} \hat{\boldsymbol{u}}_{t, h \tau} \rightarrow \boldsymbol{u}_{t t}$ weakly in $L^{2}(0, T ; \boldsymbol{V})$ for some $\boldsymbol{u}_{t t} \in L^{2}(0, T ; \boldsymbol{V})$. It holds that $\boldsymbol{u}_{t t}=\partial_{t t} \boldsymbol{u}_{\zeta \eta}$ if also $\hat{\boldsymbol{u}}_{t, h \tau} \rightarrow \partial_{t} \boldsymbol{u}_{\zeta \eta}$ weakly in $L^{2}(0, T ; \boldsymbol{V})$. From the stability result (7.3c), and the Eberlein-Šmulian theorem, $c f$., Lemma B.8, there exists a $\boldsymbol{u}_{t} \in L^{2}(0, T ; \boldsymbol{V})$ such that $\hat{\boldsymbol{u}}_{t, h \tau} \rightarrow \boldsymbol{u}_{t}$ weakly in $L^{2}(0, T ; \boldsymbol{V})$ (up to a subsequence). Employing the triangle inequality, yields

$$
\left\|\hat{\boldsymbol{u}}_{t, h \tau}-\partial_{t} \boldsymbol{u}_{\zeta \eta}\right\|_{L^{2}\left(Q_{T}\right)} \leq\left\|\hat{\boldsymbol{u}}_{t, h \tau}-\partial_{t} \hat{\boldsymbol{u}}_{h \tau}\right\|_{L^{2}\left(Q_{T}\right)}+\left\|\partial_{t} \hat{\boldsymbol{u}}_{h \tau}-\partial_{t} \boldsymbol{u}_{\zeta \eta}\right\|_{L^{2}\left(Q_{T}\right)} .
$$

Hence, due to (7.3d) and (7.5), it holds that $\boldsymbol{u}_{t}=\partial_{t} \boldsymbol{u}_{\zeta \eta}$, and consequently $\boldsymbol{u}_{t t}=\partial_{t t} \boldsymbol{u}_{\zeta \eta}$, concluding the proof.

Lemma 7.6 (Convergence of the time derivative of the pore pressure). There exists a subsequence of $\left\{\hat{p}_{\text {pore }, h \tau}\right\}_{h, \tau}$, denoted by the same index, satisfying

$$
\partial_{t} \hat{p}_{\text {pore }, h \tau} \rightarrow \partial_{t} \hat{p}_{\text {pore }}\left(\chi_{\zeta \eta}\right) \quad \text { weakly in } L^{2}\left(Q_{T}\right) .
$$

Proof. By Lemma 6.11, we have $\bar{\chi}_{h \tau} \rightarrow \chi_{\zeta \eta}$ strongly in $L^{2}\left(Q_{T}\right)$ (up to a subsequence). Hence, also $\hat{p}_{\text {pore }}\left(\bar{\chi}_{h \tau}\right) \rightarrow$ $\hat{p}_{\text {pore }}\left(\chi_{\zeta \eta}\right)$ strongly in $L^{2}\left(Q_{T}\right)$. From Lemma 7.4 , it follows $\hat{p}_{\text {pore }, h \tau} \rightarrow \hat{p}_{\text {pore }}\left(\chi_{\zeta \eta}\right)$ strongly in $L^{2}\left(Q_{T}\right)$ and $\partial_{t} \hat{p}_{\text {pore }, h \tau} \rightarrow p_{t}$ weakly in $L^{2}\left(Q_{T}\right)$ for some $p_{t} \in L^{2}\left(Q_{T}\right)$ (up to a subsequence). Consequently, $p_{t}=\partial_{t} \hat{p}_{\text {pore }}\left(\chi_{\zeta \eta}\right)$, which concludes the proof.

\subsection{Identifying a weak solution with increased regularity for $h, \tau \rightarrow 0$}

Finally, we show the limit $\left(\boldsymbol{u}_{\zeta \eta}, \chi\right)$, derived in Section 6.3, also satisfies (W5 $)_{\zeta \eta}-(\mathrm{W} 6)_{\zeta \eta}$, i.e., $\left(\boldsymbol{u}_{\zeta \eta}, \chi_{\zeta \eta}\right)$ is a weak solution for the doubly regularized unsaturated poroelasticity model with increased regularity, $c f$., Definition 4.1.

Lemma 7.7 (Limit satisfies $\left.(\mathrm{W} 1)_{\zeta \eta^{-}}(\mathrm{W} 6)_{\zeta \eta}\right)$. The limit $\left(\boldsymbol{u}_{\zeta \eta}, \chi_{\zeta \eta}\right)$, derived in Section 6.3, is a weak solution for the doubly regularized unsaturated poroelasticity model with increased regularity, cf., Definition 4.1.

Proof. The limit $\left(\boldsymbol{u}_{\zeta \eta}, \chi_{\zeta \eta}\right)$ satisfies (W1 $)_{\zeta \eta}-(\mathrm{W} 4)_{\zeta \eta}$ by Lemma 6.15. Furthermore, (W5) $)_{\zeta \eta}$ follows directly from Lemmas 7.5 and 7.6. In order to show $(\mathrm{W} 6)_{\zeta \eta}$, let $\boldsymbol{v} \in L^{2}\left(0, T ; \boldsymbol{V} \cap C^{\infty}(\Omega)^{d}\right)$. We utilize $\overline{\boldsymbol{v}}_{h \tau}$ and $\boldsymbol{v}_{h}^{n}$, as introduced in (6.14) and (6.16), respectively; again it holds that

$$
\overline{\boldsymbol{v}}_{h \tau} \rightarrow \boldsymbol{v} \text { strongly in } L^{2}(0, T ; \boldsymbol{V}) .
$$

We consider the difference of the mechanics equation (5.5a) at time steps $n$ and $n-1, n \geq 1$, tested with $\boldsymbol{v}_{h}=\boldsymbol{v}_{h}^{n}$; we obtain

$\zeta \tau^{-1} a\left(\boldsymbol{u}_{h}^{n}-2 \boldsymbol{u}_{h}^{n-1}+\boldsymbol{u}_{h}^{n-2}, \boldsymbol{v}_{h}^{n}\right)+a\left(\boldsymbol{u}_{h}^{n}-\boldsymbol{u}_{h}^{n-1}, \boldsymbol{v}_{h}^{n}\right)-\alpha\left\langle\hat{p}_{\text {pore }}\left(\chi_{h}^{n}\right)-\hat{p}_{\text {pore }}\left(\chi_{h}^{n-1}\right), \boldsymbol{\nabla} \cdot \boldsymbol{v}_{h}^{n}\right\rangle=\left\langle\boldsymbol{f}_{\text {ext }}^{n}-\boldsymbol{f}_{\text {ext }}^{n-1}, \boldsymbol{v}_{h}^{n}\right\rangle$.

Summing over $n \in\{1, \ldots, N\}$, and employing the definitions of $\overline{\boldsymbol{v}}_{h \tau}, \hat{\boldsymbol{u}}_{t, h \tau}, \hat{\boldsymbol{u}}_{h \tau}$, and $\hat{p}_{\text {pore }, h \tau}$, yields

$$
\int_{0}^{T}\left[\zeta a\left(\partial_{t} \hat{\boldsymbol{u}}_{t, h \tau}, \overline{\boldsymbol{v}}_{h \tau}\right)+a\left(\partial_{t} \hat{\boldsymbol{u}}_{h \tau}, \overline{\boldsymbol{v}}_{h \tau}\right)-\alpha\left\langle\partial_{t} \hat{p}_{\mathrm{pore}, h \tau}, \boldsymbol{\nabla} \cdot \overline{\boldsymbol{v}}_{h \tau}\right\rangle\right] \mathrm{d} t=\int_{0}^{T}\left\langle\partial_{t} \hat{\boldsymbol{f}}_{\tau}, \overline{\boldsymbol{v}}_{h \tau}\right\rangle \mathrm{d} t
$$

where $\hat{\boldsymbol{f}}_{\tau}$ denotes the piecewise linear interpolation

$$
\hat{\boldsymbol{f}}_{\mathrm{ext}, \tau}(t):=\boldsymbol{f}_{\mathrm{ext}}^{n-1}+\frac{t-t_{n-1}}{\tau}\left(\boldsymbol{f}_{\mathrm{ext}}^{n}-\boldsymbol{f}_{\mathrm{ext}}^{n-1}\right), \quad t \in\left(t_{n-1}, t_{n}\right], n \in\{1, \ldots, N\} .
$$

It holds that $\hat{\boldsymbol{f}}_{\text {ext }, \tau} \rightarrow \boldsymbol{f}_{\text {ext }}$ strongly in $L^{2}\left(0, T ; \boldsymbol{V}^{\star}\right)$ and also $\partial_{t} \hat{\boldsymbol{f}}_{\text {ext }, \tau} \rightarrow \partial_{t} \boldsymbol{f}_{\text {ext }}$ weakly in $L^{2}\left(0, T ; \boldsymbol{V}^{\star}\right)$, for $\tau \rightarrow 0$. Hence, together with the weak convergence properties of $\hat{\boldsymbol{u}}_{t, h \tau}, \hat{\boldsymbol{u}}_{h \tau}$ and $\hat{p}_{\text {pore, } h \tau}, c f$., Lemmas 7.5 and 7.6, and the strong convergence properties of the test function $\overline{\boldsymbol{v}}_{h \tau}, c f .,(7.6)$, we conclude that

$$
\int_{0}^{T}\left[\zeta a\left(\partial_{t t} \boldsymbol{u}_{\zeta \eta}, \boldsymbol{v}\right)+a\left(\partial_{t} \boldsymbol{u}_{\zeta \eta}, \boldsymbol{v}\right)-\alpha\left\langle\partial_{t} \hat{p}_{\text {pore }}\left(\chi_{\zeta \eta}\right), \boldsymbol{\nabla} \cdot \boldsymbol{v}\right\rangle\right] \mathrm{d} t=\int_{0}^{T}\left\langle\partial_{t} \boldsymbol{f}_{\text {ext }}, \boldsymbol{v}\right\rangle \mathrm{d} t
$$

for all $\boldsymbol{v} \in L^{2}\left(0, T ; \boldsymbol{V} \cap C^{\infty}(\Omega)^{d}\right)$. A density argument yields the final result. 


\section{Step 5: Limit $\zeta \rightarrow 0$ From POROViscoelasticity to POROELASTICITY}

In this section, we prove Lemma 4.6, i.e., the existence of a weak solution to the simply regularized unsaturated poroelasticity model, $c f$., Definition 4.2. For this we utilize the fact that there exists a weak solution, $\left(\boldsymbol{u}_{\zeta \eta}, \chi_{\zeta \eta}\right)$, with increased regularity for the doubly regularized unsaturated poroelasticity model, $c f$., Definition 4.1. We show that $\left\{\left(\boldsymbol{u}_{\zeta \eta}, \chi_{\zeta \eta}\right)\right\}_{\zeta}$ has a limit for $\zeta \rightarrow 0$, which is a weak solution to the simply regularized unsaturated poroelasticity model, i.e., it satisfies $(\mathrm{W} 1)_{\eta}-(\mathrm{W} 4)_{\eta}$. For this, we employ compactness arguments. The central uniform stability bound is derived utilizing (W6 $)_{\zeta \eta}$ and the non-degeneracy condition (ND3). Throughout the entire section, we assume (A0)-(A8) and (ND1)-(ND3) hold true.

\subsection{Stability estimates independent of $\zeta$}

The key ingredients for the subsequent discussion are stability estimates, which are independent of $\zeta$. As a result of the stability results derived in Section 6.2, there exists a constant $C=C\left(C^{(1)}, C^{(4)}\right)>0$ (independent of $\zeta>0$ and $\eta>0)$, such that

$$
\left\|\boldsymbol{u}_{\zeta \eta}\right\|_{L^{\infty}(0, T ; \boldsymbol{V})}^{2}+\left\|p_{\text {pore }}\left(\chi_{\zeta \eta}\right)\right\|_{L^{2}\left(Q_{T}\right)}^{2} \leq C,
$$

where Lemmas 6.6 and 6.10 yield stability for the displacement, and Lemmas 6.8 and 6.12 yield stability for the pore pressure. Further stability bounds can be obtained by exploiting the continuous nature of the balance equations and the time derivative of the mechanics equation. The following result is crucial.

Lemma 8.1 (Stability for the primal variables). There exists a constant $C^{(8)}>0$ (independent of $\zeta$ and $\eta$ ), such that

$\zeta\left\|\partial_{t} \boldsymbol{u}_{\zeta \eta}\right\|_{L^{\infty}(0, T ; \boldsymbol{V})}^{2}+\left\|\partial_{t} \boldsymbol{u}_{\zeta \eta}\right\|_{L^{2}(0, T ; \boldsymbol{V})}^{2}+\left\|\boldsymbol{\nabla} \chi_{\zeta \eta}\right\|_{L^{\infty}\left(0, T ; L^{2}(\Omega)\right)}^{2} \leq C^{(8)}\left(C_{0},\left\|\partial_{t} \boldsymbol{f}_{\mathrm{ext}}\right\|_{L^{2}\left(0, T ; \boldsymbol{V}^{\star}\right)}^{2},\left\|h_{\mathrm{ext}}\right\|_{H^{1}\left(0, T ; Q^{\star}\right)}^{2}\right)$,

where $C_{0}$ comes from $\left(\mathrm{A} 8^{\star}\right)$.

Proof. Let $\tilde{T} \in[0, T]$. Consider the flow equation (4.4b) and the mechanics equation differentiated in time (4.5), tested with $q=\mathbf{1}_{[0, \tilde{T}]} \partial_{t} \chi_{\zeta \eta}$ and $\boldsymbol{v}=\mathbf{1}_{[0, \tilde{T}]} \partial_{t} \boldsymbol{u}_{\zeta \eta}$, respectively, where $\mathbf{1}_{[0, \tilde{T}]}=\mathbf{1}_{[0, \tilde{T}]}(t), t \in[0, T]$, is the characteristic function of the time interval. Summing both equations yields

$$
\begin{aligned}
\zeta \int_{0}^{\tilde{T}} & a\left(\partial_{t t} \boldsymbol{u}_{\zeta \eta}, \partial_{t} \boldsymbol{u}_{\zeta \eta}\right) \mathrm{d} t+\int_{0}^{\tilde{T}}\left\langle\kappa_{\mathrm{abs}} \nabla \chi_{\zeta \eta}, \nabla \partial_{t} \chi_{\zeta \eta}\right\rangle \mathrm{d} t \\
& +\int_{0}^{\tilde{T}} a\left(\partial_{t} \boldsymbol{u}_{\zeta \eta}, \partial_{t} \boldsymbol{u}_{\zeta \eta}\right) \mathrm{d} t+\int_{0}^{\tilde{T}}\left\langle\partial_{t} \hat{b}_{\eta}\left(\chi_{\zeta \eta}\right), \partial_{t} \chi_{\zeta \eta}\right\rangle+\alpha \int_{0}^{\tilde{T}}\left\langle\hat{s}_{\mathrm{w}} \partial_{t} \chi_{\zeta \eta}-\partial_{t} \hat{p}_{\text {pore }}, \partial_{t} \nabla \cdot \boldsymbol{u}_{\zeta \eta}\right\rangle \\
& =\int_{0}^{\tilde{T}}\left\langle\partial_{t} \boldsymbol{f}_{\text {ext }}, \partial_{t} \boldsymbol{u}_{\zeta \eta}\right\rangle \mathrm{d} t+\int_{0}^{\tilde{T}}\left\langle h_{\text {ext }}, \partial_{t} \chi_{\zeta \eta}\right\rangle \mathrm{d} t .
\end{aligned}
$$

We discuss the individual terms separately. For the first two terms on the left hand side of (8.2), we employ the fundamental theorem of calculus

$$
\begin{aligned}
\zeta \int_{0}^{\tilde{T}} & a\left(\partial_{t t} \boldsymbol{u}_{\zeta \eta}, \partial_{t} \boldsymbol{u}_{\zeta \eta}\right) \mathrm{d} t+\int_{0}^{\tilde{T}}\left\langle\kappa_{\mathrm{abs}} \nabla \chi_{\zeta \eta}, \nabla \partial_{t} \chi_{\zeta \eta}\right\rangle \mathrm{d} t \\
& =\frac{\zeta}{2}\left\|\partial_{t} \boldsymbol{u}_{\zeta \eta}(\tilde{T})\right\|_{\boldsymbol{V}}^{2}+\frac{1}{2}\left(\left\|\sqrt{\kappa_{\mathrm{abs}}} \nabla \chi_{\zeta \eta}(\tilde{T})\right\|_{L^{2}(\Omega)}^{2}-\left\|\sqrt{\kappa_{\mathrm{abs}}} \nabla \chi_{\zeta \eta}(0)\right\|_{L^{2}(\Omega)}^{2}\right)
\end{aligned}
$$

where we used that $\partial_{t} \boldsymbol{u}_{\zeta \eta}(0)=\mathbf{0}$. This follows from the temporal derivative of the mechanics equation (4.5) and the compatibility condition for the initial conditions (3.1). 
For the remaining terms on the left hand side of (8.2), we apply the binomial identity (App. B.2), employ that $\hat{b}_{\eta}^{\prime} \geq \hat{b}^{\prime} \geq 0$ (cf., (A1)), condition (ND3), and $a(\boldsymbol{v}, \boldsymbol{v})=\|\boldsymbol{v}\|_{\boldsymbol{V}} \geq K_{\mathrm{dr}}\|\boldsymbol{\nabla} \cdot \boldsymbol{v}\|_{L^{2}(\Omega)}^{2}$ for all $\boldsymbol{v} \in \boldsymbol{V}$ with $K_{\mathrm{dr}}=\frac{2 \mu}{d}+\lambda$. We get

$$
\begin{aligned}
&\left\|\partial_{t} \boldsymbol{u}_{\zeta \eta}\right\|_{L^{2}(0, \tilde{T} ; \boldsymbol{V})}^{2}+\int_{0}^{\tilde{T}}\left\langle\partial_{t} \hat{b}_{\eta}\left(\chi_{\zeta \eta}\right), \partial_{t} \chi_{\zeta \eta}\right\rangle+\alpha \int_{0}^{\tilde{T}}\left\langle\hat{s}_{\mathrm{w}} \partial_{t} \chi_{\zeta \eta}-\partial_{t} \hat{p}_{\text {pore }}, \partial_{t} \boldsymbol{\nabla} \cdot \boldsymbol{u}_{\zeta \eta}\right\rangle \\
&=\left\|\partial_{t} \boldsymbol{u}_{\zeta \eta}\right\|_{L^{2}(0, \tilde{T} ; \boldsymbol{V})}^{2}+\int_{0}^{\tilde{T}} \int_{\Omega}\left[\left(\partial_{t} \hat{b}_{\eta} \partial_{t} \chi_{\zeta \eta}\right)^{1 / 2}+\frac{\alpha}{2} \frac{\hat{s}_{\mathrm{w}} \partial_{t} \chi_{\zeta \eta}-\partial_{t} \hat{p}_{\text {pore }}}{\left(\partial_{t} \hat{b}_{\eta} \partial_{t} \chi_{\zeta \eta}\right)^{1 / 2}} \partial_{t} \boldsymbol{\nabla} \cdot \boldsymbol{u}_{\zeta \eta}\right]^{2} \mathrm{~d} x \mathrm{~d} t \\
& \quad-\frac{\alpha^{2}}{4} \int_{0}^{\tilde{T}} \int_{\Omega} \frac{\left|\hat{s}_{\mathrm{w}} \partial_{t} \chi_{\zeta \eta}-\partial_{t} \hat{p}_{\text {pore }}\right|^{2}}{\left|\partial_{t} \hat{b}_{\eta} \partial_{t} \chi_{\zeta \eta}\right|}\left|\partial_{t} \boldsymbol{\nabla} \cdot \boldsymbol{u}_{\zeta \eta}\right|^{2} \mathrm{~d} x \mathrm{~d} t \\
& \geq \frac{1}{2}\left\|\partial_{t} \boldsymbol{u}_{\zeta \eta}\right\|_{L^{2}(0, \tilde{T} ; \boldsymbol{V})}^{2}+\frac{K_{\mathrm{dr}}}{2}\left\|\partial_{t} \boldsymbol{\nabla} \cdot \boldsymbol{u}_{\zeta \eta}\right\|_{L^{2}\left(0, \tilde{T} ; L^{2}(\Omega)\right)}^{2}-\frac{\alpha^{2}}{4} \int_{0}^{\tilde{T}} \int_{\Omega} \frac{\left(\hat{s}_{\mathrm{w}}\left(\chi_{\zeta \eta}\right)-\hat{p}_{\text {pore }}^{\prime}\left(\chi_{\zeta \eta}\right)\right)^{2}}{\hat{b}^{\prime}\left(\chi_{\zeta \eta}\right)}\left|\partial_{t} \boldsymbol{\nabla} \cdot \boldsymbol{u}_{\zeta \eta}\right|^{2} \mathrm{~d} x \mathrm{~d} t \\
& \geq \frac{1}{2}\left\|\partial_{t} \boldsymbol{u}_{\zeta \eta}\right\|_{L^{2}(0, \tilde{T} ; \boldsymbol{V})}^{2} .
\end{aligned}
$$

For the first term on the right hand side of (8.2), we apply the Cauchy-Schwarz and Young's inequality

$$
\int_{0}^{\tilde{T}}\left\langle\partial_{t} \boldsymbol{f}_{\mathrm{ext}}, \partial_{t} \boldsymbol{u}_{\zeta \eta}\right\rangle \mathrm{d} t \leq\left\|\partial_{t} \boldsymbol{f}_{\mathrm{ext}}\right\|_{L^{2}\left(0, \tilde{T} ; \boldsymbol{V}^{\star}\right)}^{2}+\frac{1}{4}\left\|\partial_{t} \boldsymbol{u}_{\zeta \eta}\right\|_{L^{2}(0, \tilde{T} ; \boldsymbol{V})}^{2} .
$$

For the second term on the right hand side of (8.2), we apply integration by parts, a Cauchy-Schwarz inequality and Young's inequality, a Poincaré inequality and a Sobolev embedding, involving the constants $C_{\Omega, \mathrm{P}}$ and $C_{\mathrm{T}, \text { Sob }}$, as well as $(\mathrm{A} 6)$. This gives

$$
\begin{aligned}
\int_{0}^{\tilde{T}}\left\langle h_{\mathrm{ext}}, \partial_{t} \chi_{\zeta \eta}\right\rangle \mathrm{d} t= & \left\langle h_{\mathrm{ext}}(\tilde{T}), \chi_{\zeta \eta}(\tilde{T})\right\rangle-\left\langle h_{\mathrm{ext}}(0), \chi(0)\right\rangle-\int_{0}^{\tilde{T}}\left\langle\partial_{t} h_{\mathrm{ext}}, \chi_{\zeta \eta}\right\rangle \mathrm{d} t \\
\leq & \frac{C_{\Omega, \mathrm{P}}^{2}}{\kappa_{\mathrm{m}, \mathrm{abs}}}\left(\left\|h_{\mathrm{ext}}(\tilde{T})\right\|_{L^{2}(\Omega)}^{2}+\left\|h_{\mathrm{ext}}(0)\right\|_{L^{2}(\Omega)}^{2}+\left\|\partial_{t} h_{\mathrm{ext}}\right\|_{L^{2}\left(Q_{\tilde{T}}\right)}^{2}\right) \\
& +\frac{\kappa_{\mathrm{m}, \mathrm{abs}}}{4 C_{\Omega, \mathrm{P}}^{2}}\left(\left\|\chi_{\zeta \eta}(\tilde{T})\right\|_{L^{2}(\Omega)}^{2}+\left\|\chi_{\zeta \eta}(0)\right\|_{L^{2}(\Omega)}^{2}+\left\|\chi_{\zeta \eta}\right\|_{L^{2}\left(Q_{\tilde{T}}\right)}^{2}\right) \\
\leq & \frac{3\left(C_{\mathrm{T}, \mathrm{Sob}} C_{\Omega, \mathrm{P}}\right)^{2}}{\kappa_{\mathrm{m}, \mathrm{abs}}}\left\|h_{\mathrm{ext}}\right\|_{H^{1}\left(0, \tilde{T} ; Q^{\star}\right)}^{2} \\
& +\frac{1}{4}\left(\left\|\sqrt{\kappa_{\mathrm{abs}}} \nabla \chi_{\zeta \eta}(\tilde{T})\right\|_{L^{2}(\Omega)}^{2}+\left\|\sqrt{\kappa_{\mathrm{abs}}} \nabla \chi_{\zeta \eta}(0)\right\|_{L^{2}(\Omega)}^{2}+\int_{0}^{\tilde{T}}\left\|\sqrt{\kappa_{\mathrm{abs}}} \nabla \chi_{\zeta \eta}\right\|_{L^{2}(\Omega)}^{2} \mathrm{~d} t\right) .
\end{aligned}
$$

Altogether, (8.2) becomes

$$
\begin{aligned}
\frac{\zeta}{2}\left\|\partial_{t} \boldsymbol{u}_{\zeta \eta}(\tilde{T})\right\|_{L^{2}(0, \tilde{T} ; \boldsymbol{V})}^{2}+\frac{1}{4}\left\|\sqrt{\kappa_{\mathrm{abs}}} \boldsymbol{\nabla} \chi_{\zeta \eta}(\tilde{T})\right\|_{L^{2}(\Omega)}^{2}+\frac{1}{4}\left\|\partial_{t} \boldsymbol{u}_{\zeta \eta}\right\|_{L^{2}(0, \tilde{T} ; \boldsymbol{V})}^{2} \\
\leq \leq \frac{3}{4}\left\|\sqrt{\kappa_{\mathrm{abs}}} \nabla \chi_{\zeta \eta}(0)\right\|_{L^{2}(\Omega)}^{2}+\left\|\partial_{t} \boldsymbol{f}_{\mathrm{ext}}\right\|_{L^{2}\left(0, \tilde{T} ; \boldsymbol{V}^{\star}\right)}^{2} \\
\quad+\frac{3\left(C_{\mathrm{T}, \mathrm{Sob}} C_{\Omega, \mathrm{P}}\right)^{2}}{\kappa_{\mathrm{m}, \mathrm{abs}}}\left\|h_{\mathrm{ext}}\right\|_{H^{1}\left(0, \tilde{T} ; Q^{\star}\right)}^{2}+\frac{1}{4} \int_{0}^{\tilde{T}}\left\|\sqrt{\kappa_{\mathrm{abs}}} \boldsymbol{\nabla} \chi_{\zeta \eta}\right\|_{L^{2}(\Omega)}^{2} \mathrm{~d} t .
\end{aligned}
$$

A Grönwall inequality, and the general choice of $\tilde{T}$, yields the assertion under the given assumptions.

The previous stability estimate allows for deriving revised stability estimates in the previous section. 
Lemma 8.2 (Stability for the energy $\hat{B}_{\eta}$ ). There exists a constant $C^{(9)}>0$ (independent of $\zeta, \eta$ ), such that

$$
\left\|\hat{B}_{\eta}\left(\chi_{\zeta \eta}\right)\right\|_{L^{\infty}\left(0, T ; L^{1}(\Omega)\right)} \leq C^{(9)}\left(C_{0}, C^{(8)}\right),
$$

where $C^{(8)}$ is the stability constant from Lemma 8.1, and $C_{0}$ is the stability constant from $\left(\mathrm{A} 8^{\star}\right)$.

Proof. Testing the flow equation (4.4b) with $q=\chi_{\zeta \eta}$, yields

$$
\int_{0}^{T}\left\langle\partial_{t} \hat{b}_{\eta}\left(\chi_{\zeta \eta}\right), \chi_{\zeta \eta}\right\rangle \mathrm{d} t+\int_{0}^{T}\left\|\boldsymbol{\nabla} \chi_{\zeta \eta}\right\|_{\kappa_{\mathrm{abs}}}^{2} \mathrm{~d} t=\int_{0}^{T}\left\langle h_{\mathrm{ext}}, \chi_{\zeta \eta}\right\rangle \mathrm{d} t-\alpha \int_{0}^{T}\left\langle\hat{s}_{\mathrm{w}} \partial_{t} \boldsymbol{\nabla} \cdot \boldsymbol{u}_{\zeta \eta}, \chi_{\zeta \eta}\right\rangle \mathrm{d} t
$$

For the first term on the left hand side, we apply a property of $\hat{B}_{\eta}, c f$. , Lemma B.12,

$$
\int_{0}^{T}\left\langle\partial_{t} \hat{b}_{\eta}\left(\chi_{\zeta \eta}\right), \chi_{\zeta \eta}\right\rangle \mathrm{d} t=\left\|\hat{B}_{\eta}\left(\chi_{\zeta \eta}(T)\right)\right\|_{L^{1}(\Omega)}-\left\|\hat{B}_{\eta}\left(\chi_{0}\right)\right\|_{L^{1}(\Omega)} .
$$

On the right hand side, we apply the Cauchy-Schwarz inequality, Young's inequality, a Poincaré inequality (introducing $C_{\Omega, \mathrm{P}}$ ) and (A6), and obtain

$$
\left\|\hat{B}_{\eta}\left(\chi_{\zeta \eta}(T)\right)\right\|_{L^{1}(\Omega)}+\frac{1}{2} \int_{0}^{T}\left\|\nabla \chi_{\zeta \eta}\right\|_{\kappa_{\text {abs }}}^{2} \mathrm{~d} t \leq\left\|\hat{B}_{\eta}\left(\chi_{0}\right)\right\|_{L^{1}(\Omega)}+\frac{C_{\Omega, \mathrm{P}}^{2}}{\kappa_{\mathrm{m}, \mathrm{abs}}}\left(\left\|h_{\mathrm{ext}}\right\|_{L^{2}\left(0, T ; Q^{\star}\right)}^{2}+\alpha^{2}\left\|\partial_{t} \boldsymbol{\nabla} \cdot \boldsymbol{u}_{\zeta \eta}\right\|_{L^{2}\left(Q_{T}\right)}^{2}\right) .
$$

Finally, the thesis follows from Lemma 8.1.

Lemma 8.3 (Stability for the temporal change of $\hat{b}_{\eta}$ ). There exists a constant $C^{(10)}>0$ (independent of $\zeta, \eta$ ), such that

$$
\sup _{0 \neq q \in L^{2}(0, T ; Q)} \frac{\int_{0}^{T}\left\langle\partial_{t} \hat{b}_{\eta}\left(\chi_{\zeta \eta}\right), q\right\rangle \mathrm{d} t}{\|\nabla q\|_{L^{2}\left(Q_{T}\right)}} \leq C^{(10)}\left(C^{(8)}\right)
$$

where $C^{(8)}$ is the stability constant from Lemma 8.1.

Proof. The proof is similar to the proof of Lemma 6.5. However, this time, we exploit

$$
\left\|\partial_{t} \boldsymbol{\nabla} \cdot \boldsymbol{u}_{\zeta \eta}\right\|_{L^{2}\left(Q_{T}\right)} \leq \frac{1}{K_{\mathrm{dr}}^{1 / 2}}\left\|\partial_{t} \boldsymbol{u}_{\zeta \eta}\right\|_{L^{2}(0, T ; \boldsymbol{V})} \leq \frac{C^{(8)}}{K_{\mathrm{dr}}^{1 / 2}}
$$

by Lemma 8.1. Thus, we can drop the dependence on $\zeta$.

We will require to show strong convergence of the Kirchhoff pressure. Having that in mind, we conclude with a stability estimate for $\partial_{t} \chi_{\zeta \eta}$. We note, this is the only stability estimate in this section, requiring the regularizing growth condition $(\mathrm{A} 1)_{\eta}$.

Lemma 8.4 (Stability estimate for the temporal change of the Kirchhoff pressure). There exists a constant $C_{\eta}^{(11)}>0$ (independent of $\zeta$ ), such that

$$
\left\|\partial_{t} \chi_{\zeta \eta}\right\|_{L^{2}\left(Q_{T}\right)}^{2} \leq C_{\eta}^{(11)}\left(b_{\chi, \mathrm{m}}^{-1} C_{0}, b_{\chi, \mathrm{m}}^{-2} C^{(8)}\right)
$$

where $C^{(8)}$ is the stability constant from Lemma 8.1, $b_{\chi, \mathrm{m}}$ is from $\left(\mathrm{A} 1^{\star}\right)$, and $C_{0}$ is from $\left(\mathrm{A} 8^{\star}\right)$. 
Proof. We repeat parts of the proof of Lemma 8.1. We test the flow equation (4.4b) with $q=\partial_{t} \chi_{\zeta \eta}$ and apply $(\mathrm{A} 1)_{\eta}$ and the Cauchy-Schwarz inequality; we obtain

$$
\begin{aligned}
b_{\chi, \mathrm{m}} & \left\|\partial_{t} \chi_{\zeta \eta}\right\|_{L^{2}\left(Q_{T}\right)}^{2}+\frac{1}{2}\left\|\sqrt{\kappa_{\mathrm{abs}}} \nabla \chi_{\zeta \eta}(T)\right\|_{L^{2}(\Omega)}^{2} \\
& \leq \int_{0}^{T}\left\langle\partial_{t} \hat{b}_{\eta}\left(\chi_{\zeta \eta}\right), \partial_{t} \chi_{\zeta \eta}\right\rangle \mathrm{d} t+\frac{1}{2}\left\|\sqrt{\kappa_{\mathrm{abs}}} \nabla \chi_{\zeta \eta}(T)\right\|_{L^{2}(\Omega)}^{2} \\
& =\frac{1}{2}\left\|\sqrt{\kappa_{\mathrm{abs}}} \boldsymbol{\nabla} \chi_{\zeta \eta}(0)\right\|_{L^{2}(\Omega)}^{2}+\int_{0}^{T}\left(\left\langle h_{\mathrm{ext}}, \partial_{t} \chi_{\zeta \eta}\right\rangle-\alpha\left\langle\hat{s}_{\mathrm{w}} \partial_{t} \boldsymbol{\nabla} \cdot \boldsymbol{u}_{\zeta \eta}, \partial_{t} \chi_{\zeta \eta}\right\rangle\right) \mathrm{d} t \\
& \leq \frac{1}{2}\left\|\sqrt{\kappa_{\mathrm{abs}}} \boldsymbol{\nabla} \chi_{\zeta \eta}(0)\right\|_{L^{2}(\Omega)}^{2}+\frac{1}{b_{\chi, \mathrm{m}}}\left(\left\|h_{\mathrm{ext}}\right\|_{L^{2}\left(0, T ; Q^{\star}\right)}^{2}+\alpha^{2}\left\|\partial_{t} \boldsymbol{\nabla} \cdot \boldsymbol{u}_{\zeta \eta}\right\|_{L^{2}\left(Q_{T}\right)}^{2}\right)+\frac{b_{\chi, \mathrm{m}}}{2}\left\|\partial_{t} \chi_{\zeta \eta}\right\|_{L^{2}\left(Q_{T}\right)}^{2} .
\end{aligned}
$$

After rearranging terms, applying the regularity of the data, and applying Lemma 8.1, the assertion follows.

\subsection{Relative (weak) compactness for $\zeta \rightarrow 0$}

We utilize the stability results from the previous section to conclude relative compactness.

Lemma 8.5 (Convergence of the primary variables). There exist subsequences of $\left\{\boldsymbol{u}_{\zeta \eta}\right\}_{\zeta}$ and $\left\{\chi_{\zeta \eta}\right\}_{\zeta}$, denoted by the same subscript, and there exist $\boldsymbol{u}_{\eta} \in H^{1}(0, T ; \boldsymbol{V})$ and $\chi_{\eta} \in H^{1}\left(0, T ; L^{2}(\Omega)\right) \cap L^{\infty}(0, T ; Q)$ such that for $\zeta \rightarrow 0$

$$
\begin{aligned}
\boldsymbol{u}_{\zeta \eta} & \rightarrow \boldsymbol{u}_{\eta} & & \text { weakly in } H^{1}(0, T ; \boldsymbol{V}), \\
\zeta \partial_{t} \boldsymbol{u}_{\zeta \eta} & \rightarrow \mathbf{0} & & \text { strongly in } L^{2}(0, T ; \boldsymbol{V}), \\
\chi_{\zeta \eta} & \rightarrow \chi_{\eta} & & \text { strongly in } L^{2}\left(Q_{T}\right), \\
\chi_{\zeta \eta} & \rightarrow \chi_{\eta} & & \text { weakly in } L^{\infty}(0, T ; Q), \\
\partial_{t} \chi_{\zeta \eta} & \rightarrow \partial_{t} \chi_{\eta} & & \text { weakly in } L^{2}\left(Q_{T}\right) .
\end{aligned}
$$

Proof. The proof follows standard arguments based on the Eberlein-Šmulian theorem, $c f$. , Lemma B.8, the Aubin-Lions lemma, cf., Lemma B.9, and the stability results for $\boldsymbol{u}_{\zeta \eta}, c f$. ., Lemma 8.1 and (8.1), as well as the stability results for $\chi_{\zeta \eta}, c f$., Lemmas 8.1 and 8.4. In particular, for (8.3b), we employ the uniform stability result from Lemma 8.1 yielding

$$
\left|\int_{0}^{T} \zeta a\left(\partial_{t} \boldsymbol{u}_{\zeta \eta}, \boldsymbol{v}\right) \mathrm{d} t\right| \leq \zeta C^{(8)}\|\boldsymbol{v}\|_{L^{2}(0, T ; \boldsymbol{V})} \rightarrow 0 \quad \text { for } \zeta \rightarrow 0
$$

for all fixed $\boldsymbol{v} \in L^{2}(0, T ; \boldsymbol{V})$.

Lemma 8.6 (Convergence of the coupling terms). There exist subsequences of $\left\{\boldsymbol{u}_{\zeta \eta}\right\}_{\zeta}$ and $\left\{\chi_{\zeta \eta}\right\}_{\zeta}$, denoted by the same subscript, satisfying for $\zeta \rightarrow 0$

$$
\begin{aligned}
\hat{p}_{\text {pore }}\left(\chi_{\zeta \eta}\right) & \rightarrow \hat{p}_{\text {pore }}\left(\chi_{\eta}\right) & & \text { weakly in } L^{2}\left(Q_{T}\right), \\
\hat{s}_{\mathrm{w}}\left(\chi_{\zeta \eta}\right) \partial_{t} \boldsymbol{\nabla} \cdot \boldsymbol{u}_{\zeta \eta} & \rightarrow \hat{s}_{\mathrm{w}}\left(\chi_{\eta}\right) \partial_{t} \boldsymbol{\nabla} \cdot \boldsymbol{u}_{\eta} & & \text { weakly in } L^{2}\left(Q_{T}\right) .
\end{aligned}
$$

Proof. The proof is analogous to the proof of Lemma 6.12. Essentially, first, one has to utilize stability estimates together with the Eberlein-Šmulian theorem, $c f$., Lemma B.8; second, continuity properties of the non-linearities have to be employed together with the convergence of $\left\{\boldsymbol{u}_{\zeta \eta}\right\}_{\zeta}$ and $\left\{\chi_{\zeta \eta}\right\}_{\zeta}, c f$., Lemma 8.5. We note that for (8.4a) the stability result (8.1) has to be utilized. 
Lemma 8.7 (Initial conditions for the fluid flow). Up to subsequences it holds for $\zeta \rightarrow 0$

$$
\partial_{t} \hat{b}_{\eta}\left(\chi_{\zeta \eta}\right) \rightarrow \partial_{t} \hat{b}_{\eta}\left(\chi_{\eta}\right) \quad \text { weakly in } L^{2}\left(0, T ; Q^{\star}\right)
$$

where $\partial_{t} \hat{b}_{\eta}\left(\chi_{\eta}\right)$ is understood in the sense of $(\mathrm{W} 2)_{\eta}$.

Proof. The proof is similar to the proof of Lemma 6.13. In particular, an analogous argument implies that $\left\|\hat{b}_{\eta}\left(\chi_{\zeta \eta}\right)\right\|_{L^{\infty}\left(0, T ; L^{1}(\Omega)\right.}$ is uniformly bounded wrt. $\zeta$, and therefore

$$
\hat{b}_{\eta}\left(\chi_{\zeta \eta}\right) \rightarrow \hat{b}_{\eta}\left(\chi_{\eta}\right) \quad \text { strongly in } L^{1}\left(0, T ; L^{1}(\Omega)\right) \text { for } \zeta \rightarrow 0,
$$

(up to a subsequence). Furthermore, by Lemma 8.3 and the Eberlein-Šmulian theorem, $c f$. , Lemma B.8, there exists some $b_{t} \in L^{2}\left(0, T ; Q^{\star}\right)$ such that $\partial_{t} \hat{b}_{\eta}\left(\chi_{\zeta \eta}\right) \rightarrow b_{t}$ weakly in $L^{2}\left(0, T ; Q^{\star}\right)$ (up to a subsequence). We can identify $b_{t}=\partial_{t} b\left(\chi_{\eta}\right)$ by showing $(\mathrm{W} 2)_{\eta}$. For this we utilize $(\mathrm{W} 2)_{\zeta \eta}$. For $q \in L^{2}(0, T ; Q) \cap W^{1,1}\left(0, T: L^{\infty}(\Omega)\right)$ and $q(T)=0$, it holds that

$$
\int_{0}^{T}\left\langle\partial_{t} \hat{b}_{\eta}\left(\chi_{\zeta \eta}\right), q\right\rangle \mathrm{d} t=\int_{0}^{T}\left\langle\hat{b}_{\eta}\left(\chi_{0}\right)-\hat{b}_{\eta}\left(\chi_{\zeta \eta}\right), \partial_{t} q\right\rangle \mathrm{d} t .
$$

The assertion follows immediately for $\zeta \rightarrow 0$, using (8.6), and the dominated convergence theorem.

Lemma 8.8 (Initial condition for the mechanical displacement). The displacement $\boldsymbol{u}_{\eta}$ satisfies (W3) ${ }_{\eta}$.

Proof. Using the uniform stability bound for $\left\{\partial_{t} \boldsymbol{u}_{\zeta \eta}\right\}_{\zeta}$ by Lemma 8.1 and the weak convergence $\boldsymbol{u}_{\zeta \eta} \rightarrow \boldsymbol{u}_{\eta}$ weakly in $L^{2}(0, T ; \boldsymbol{V})$ (up to a subsequence) by Lemma 8.5, standard compactness arguments yield

$$
\partial_{t} \boldsymbol{u}_{\zeta \eta} \rightarrow \partial_{t} \boldsymbol{u}_{\eta} \quad \text { weakly in } L^{2}(0, T ; \boldsymbol{V})
$$

(up to a subsequence). Hence, $\zeta \rightarrow 0$ of $(\mathrm{W} 3)_{\zeta \eta}$ yields $(\mathrm{W} 3)_{\eta}$.

\subsection{Identifying a weak solution for $\zeta \rightarrow 0$}

Finally, we show the limit $\left(\boldsymbol{u}_{\eta}, \chi_{\eta}\right)$, introduced above, is a weak solution of the simply regularized unsaturated poroelasticity model.

Lemma 8.9 (Limit satisfies $\left.(\mathrm{W} 1)_{\eta}-(\mathrm{W} 4)_{\eta}\right)$. The limit $\left(\boldsymbol{u}_{\eta}, \chi_{\eta}\right)$, derived in Section 8.2, is a weak solution of the simply regularized unsaturated poroelasticity model, cf., Definition 4.2.

Proof. The limit $\left(\boldsymbol{u}_{\eta}, \chi_{\eta}\right)$ satisfies $(\mathrm{W} 1)_{\eta}-(\mathrm{W} 3)_{\eta}$ by Lemmas 8.5-8.8. It remains to show $(\mathrm{W} 4)_{\eta}$, i.e., that $\left(\boldsymbol{u}_{\eta}, \chi_{\eta}\right)$ satisfies the balance equations (4.4) for $\zeta=0$. By definition, the sequence $\left(\boldsymbol{u}_{\zeta \eta}, \chi_{\zeta \eta}\right)$ satisfies $(\mathrm{W} 4)_{\zeta \eta}$ for $\zeta>0$. Utilizing the weak convergence results, $c f$., Lemmas 8.5 and 8.6, $(\mathrm{W} 4)_{\eta}$ follows directly for $\zeta \rightarrow 0$.

Remark 8.10 (Existence of a weak solution for a compressible system). If compressibility is present either for the fluid or the solid grains, the regularizing property $\left(\mathrm{A} 1^{\star}\right)$ is fulfilled even for $\eta=0$. For instance, for $b$ as in (2.6) with the equivalent pore pressure and the van Genuchten-Mualem model, it holds that $b_{\chi, \mathrm{m}}=\phi_{0} c_{\mathrm{w}}+\frac{1}{N}$, $c f$., Appendix A. Consequently, the limit $\left(\boldsymbol{u}_{\eta}, \chi_{\eta}\right)$ in Lemma 8.9 is also well-defined for $\eta=0$. In particular, it is a weak solution of (2.12)-(2.15), cf., Definition 3.1.

\section{STEP 6: Limit $\eta \rightarrow 0$ IN THE INCOMPRESSIBLE CASE}

In this section, we show the main result, Theorem 3.4, for the more demanding case: the presence of an incompressible fluid and incompressible solid grains. Otherwise, by Remark 8.10 the main result of this paper follows already. In the incompressible case, $b$ as in (2.6) is monotone but with $\hat{b}^{\prime}=0$ on a part of the domain with non-zero measure. Under the use of regularization with $\eta>0$, it holds that $b_{\chi, \mathrm{m}}=\eta$. In the following, we prove that the limit of $\left\{\left(\boldsymbol{u}_{\eta}, \chi_{\eta}\right)\right\}_{\eta}$ exists for $\eta \rightarrow 0$, and that it is a weak solution of (2.12)-(2.15) according to Definition 3.1. Throughout the entire section, we assume (A0)-(A8) and (ND1)-(ND3) hold true. 


\subsection{Stability estimates independent of $\eta$}

In Section 8, many stability bounds are independent of $\eta$. To summarize, there exists a constant $C>0$ (independent of $\eta$ ) such that

$$
\begin{aligned}
& \left\|\boldsymbol{u}_{\eta}\right\|_{H^{1}(0, T ; \boldsymbol{V})}+\left\|\chi_{\eta}\right\|_{L^{\infty}\left(0, T ; H_{0}^{1}(\Omega)\right)}+\left\|\hat{p}_{\text {pore }}\left(\chi_{\eta}\right)\right\|_{L^{2}\left(Q_{T}\right)} \\
& \quad+\left\|\hat{B}_{\eta}\left(\chi_{\eta}\right)\right\|_{L^{\infty}\left(0, T ; L^{1}(\Omega)\right)}+\left\|\partial_{t} \hat{b}_{\eta}\left(\chi_{\eta}\right)\right\|_{L^{2}\left(0, T ; H^{-1}(\Omega)\right)} \leq C .
\end{aligned}
$$

The only stability bound depending on $\eta$ is the one of $\partial_{t} \chi_{\eta}, c f$., Lemma 8.4. We recall, there exists a constant $C_{\eta}>0$, depending on $\eta$, satisfying

$$
\left\|\partial_{t} \chi_{\eta}\right\|_{L^{2}\left(Q_{T}\right)} \leq C_{\eta}
$$

In order to conclude that $\left(\boldsymbol{u}_{\eta}, \chi_{\eta}\right)$ converges towards a weak solution of the unsaturated poroelasticity model, it will be sufficient to replace the stability result (9.2) by a uniform stability estimate. The remaining discussion for $\eta \rightarrow 0$ can be done along the lines of Sections 8.2 and 8.3 .

In the following, we prove a uniform stability bound replacing (9.2) in two steps. We show that the temporal derivative of the mechanics equation, i.e., (W5) $\zeta \eta$ for $\zeta=0$, is well-defined; and then we use an inf-sup argument and the uniform stability estimate (9.1).

Lemma 9.1 (Temporal derivative of the mechanics equation). It holds for all $\boldsymbol{v} \in L^{2}(0, T ; \boldsymbol{V})$

$$
\int_{0}^{T} a\left(\partial_{t} \boldsymbol{u}_{\eta}, \boldsymbol{v}\right) \mathrm{d} t-\int_{0}^{T} \alpha\left\langle\partial_{t} \hat{p}_{\text {pore }}\left(\chi_{\eta}\right), \boldsymbol{\nabla} \cdot \boldsymbol{v}\right\rangle \mathrm{d} t=\int_{0}^{T}\left\langle\partial_{t} \boldsymbol{f}_{\text {ext }}, \boldsymbol{v}\right\rangle \mathrm{d} t .
$$

Proof. First, we argue that the mechanics equation (3.2a) holds pointwise on $[0, T]$. Let $\boldsymbol{v} \in L^{2}(0, T ; \boldsymbol{V}) \cap$ $C^{\infty}(0, T ; \boldsymbol{V})$. By Lemma 8.9 , it holds that

$$
\int_{0}^{T} a\left(\boldsymbol{u}_{\eta}, \boldsymbol{v}\right) \mathrm{d} t-\int_{0}^{T} \alpha\left\langle\hat{p}_{\text {pore }}\left(\chi_{\eta}\right), \nabla \cdot \boldsymbol{v}\right\rangle \mathrm{d} t=\int_{0}^{T}\left\langle\boldsymbol{f}_{\text {ext }}, \boldsymbol{v}\right\rangle \mathrm{d} t .
$$

By the fundamental lemma of calculus of variations it follows a.e. in $[0, T]$

$$
a\left(\boldsymbol{u}_{\eta}, \boldsymbol{v}\right)-\alpha\left\langle\hat{p}_{\text {pore }}\left(\chi_{\eta}\right), \boldsymbol{\nabla} \cdot \boldsymbol{v}\right\rangle=\left\langle\boldsymbol{f}_{\text {ext }}, \boldsymbol{v}\right\rangle, \quad \text { for all } \boldsymbol{v} \in \boldsymbol{V} .
$$

Applying a standard embedding for Bochner spaces,[18] we can assume wlog. that $\boldsymbol{u}_{\eta} \in C(0, T ; \boldsymbol{V})$ and $\hat{p}_{\text {pore }}\left(\chi_{\eta}\right) \in C\left(0, T ; L^{2}(\Omega)\right)$, as $\partial_{t} \boldsymbol{u}_{\eta} \in L^{2}(0, T ; \boldsymbol{V})$ and $\partial_{t} \hat{p}_{\text {pore }}\left(\chi_{\eta}\right) \in L^{2}\left(Q_{T}\right)$ by $(9.2)$ and assumption (ND2). Hence, (9.4) holds pointwise on $[0, T]$.

Now we show (9.3). Let $\boldsymbol{v} \in L^{2}(0, T ; \boldsymbol{V}) \cap C^{\infty}(0, T ; \boldsymbol{V})$. By Lemma 8.9, it holds that

$$
\int_{0}^{T} a\left(\boldsymbol{u}_{\eta}, \partial_{t} \boldsymbol{v}\right) \mathrm{d} t-\alpha \int_{0}^{T}\left\langle\hat{p}_{\text {pore }}\left(\chi_{\eta}\right), \boldsymbol{\nabla} \cdot \partial_{t} \boldsymbol{v}\right\rangle \mathrm{d} t=\int_{0}^{T}\left\langle\boldsymbol{f}_{\text {ext }}, \partial_{t} \boldsymbol{v}\right\rangle \mathrm{d} t .
$$

Since $\partial_{t} \boldsymbol{u}_{\eta} \in L^{2}(0, T ; \boldsymbol{V}), \partial_{t} \hat{p}_{\text {pore }}\left(\chi_{\eta}\right) \in L^{2}\left(Q_{T}\right)$ and $\partial_{t} \boldsymbol{f}_{\text {ext }} \in L^{2}\left(0, T ; \boldsymbol{V}^{\star}\right)$, integration by parts is well-defined. Together with (9.4), we obtain

$$
\int_{0}^{T} a\left(\partial_{t} \boldsymbol{u}_{\eta}, \boldsymbol{v}\right) \mathrm{d} t-\alpha \int_{0}^{T}\left\langle\partial_{t} \hat{p}_{\text {pore }}\left(\chi_{\eta}\right), \boldsymbol{\nabla} \cdot \boldsymbol{v}\right\rangle \mathrm{d} t=\int_{0}^{T}\left\langle\partial_{t} \boldsymbol{f}_{\text {ext }}, \boldsymbol{v}\right\rangle \mathrm{d} t .
$$

The assertion follows after applying a density argument allowing for arbitrary test functions in $L^{2}(0, T ; \boldsymbol{V})$ in (9.3). 
Lemma 9.2 (Stability estimate for the temporal derivative of the Kirchhoff pressure). There exists a constant $C^{(12)}>0$ (independent of $\eta$ ) such that

$$
\left\|\partial_{t} \chi_{\eta}\right\|_{L^{2}\left(Q_{T}\right)} \leq C^{(12)} .
$$

Proof. We show that $\partial_{t} \hat{p}_{\text {pore }}\left(\chi_{\eta}\right)$ is uniformly bounded in $L^{2}\left(Q_{T}\right)$. The assertion follows then from the assumption (ND2), as

$$
\left\|\partial_{t} \chi_{\eta}\right\|_{L^{2}\left(Q_{T}\right)} \leq C_{\mathrm{ND}, 2}\left\|\partial_{t} \hat{p}_{\text {pore }}\left(\chi_{\eta}\right)\right\|_{L^{2}\left(Q_{T}\right)} .
$$

By Lemma 9.1, the time derivative of the mechanics equation is well-defined, $c f .$, (9.3). Using a standard inf-sup argument (introducing the constant $C_{\Omega \text {,is }}$ ), $c f$., Lemma B.11, it follows from (9.3) that

$$
\left\|\partial_{t} \hat{p}_{\text {pore }}\left(\chi_{\eta}\right)\right\|_{L^{2}\left(Q_{T}\right)} \leq C_{\Omega, \text { is }}\left(\left\|\partial_{t} \boldsymbol{u}_{\eta}\right\|_{L^{2}(0, T ; \boldsymbol{V})}+\left\|\partial_{t} \boldsymbol{f}_{\text {ext }}\right\|_{L^{2}\left(0, T ; \boldsymbol{V}^{\star}\right)}\right) .
$$

Since $\left\|\partial_{t} \boldsymbol{u}_{\eta}\right\|_{L^{2}(0, T ; \boldsymbol{V})}$ is uniformly bounded by (9.1), and (A7) holds true, $\left\|\partial_{t} \hat{p}_{\text {pore }}\left(\chi_{\eta}\right)\right\|_{L^{2}\left(Q_{T}\right)}$ is uniformly bounded, which concludes the proof.

\subsection{Relative (weak) compactness for $\eta \rightarrow 0$}

Using the same line of argumentation as used in Section 8.2, we can discuss the limit process $\eta \rightarrow 0$.

Lemma 9.3 (Convergence of the primary variables). There exist subsequences of $\left\{\boldsymbol{u}_{\eta}\right\}_{\eta}$ and $\left\{\chi_{\eta}\right\}_{\eta}$, denoted by the same subscript, and there exist $\boldsymbol{u} \in H^{1}(0, T ; \boldsymbol{V})$ and $\chi \in H^{1}\left(0, T ; L^{2}(\Omega)\right) \cap L^{\infty}(0, T ; Q)$ such that for $\eta \rightarrow 0$

$$
\begin{array}{clrl}
\boldsymbol{u}_{\eta} & \rightarrow \boldsymbol{u} & & \text { weakly in } H^{1}(0, T ; \boldsymbol{V}), \\
\chi_{\eta} & \rightarrow \chi & & \text { strongly in } L^{2}\left(Q_{T}\right), \\
\chi_{\eta} & \rightarrow \chi & & \text { weakly in } L^{\infty}(0, T ; Q), \\
\partial_{t} \chi_{\eta} & \rightarrow \partial_{t} \chi & & \text { weakly in } L^{2}\left(Q_{T}\right) .
\end{array}
$$

Proof. The proof is analogous to the proofs of Lemma 8.5.

Lemma 9.4 (Convergence of the coupling terms). There exist subsequences of $\left\{\boldsymbol{u}_{\eta}\right\}_{\eta}$ and $\left\{\chi_{\eta}\right\}_{\eta}$, denoted by the same subscript, satisfying for $\eta \rightarrow 0$

$$
\begin{aligned}
& \hat{p}_{\text {pore }}\left(\chi_{\eta}\right) \rightarrow \hat{p}_{\text {pore }}(\chi) \quad \text { weakly in } L^{2}\left(Q_{T}\right), \\
& \hat{s}_{\mathrm{w}}\left(\chi_{\eta}\right) \partial_{t} \boldsymbol{\nabla} \cdot \boldsymbol{u}_{\eta} \rightarrow \hat{s}_{\mathrm{w}}(\chi) \partial_{t} \boldsymbol{\nabla} \cdot \boldsymbol{u} \quad \text { weakly in } L^{2}\left(Q_{T}\right) \text {. }
\end{aligned}
$$

Proof. The proof is analogous to the proof of Lemma 8.6.

Lemma 9.5 (Initial conditions for the fluid flow). There exist a subsequence of $\left\{\chi_{\eta}\right\}_{\eta}$, denoted by the same subscript, satisfying

$$
\partial_{t} \hat{b}_{\eta}\left(\chi_{\eta}\right) \rightarrow \partial_{t} \hat{b}(\chi) \quad \text { weakly in } L^{2}\left(0, T ; Q^{\star}\right)
$$

where $\partial_{t} \hat{b}(\chi)$ is understood in the sense of (W2).

Proof. The proof is analogous to the proof of Lemma 8.7. We only stress that due to construction of $\hat{b}_{\eta}$, one can show that if $\chi_{\eta} \rightarrow \chi$ in $L^{2}\left(Q_{T}\right)$, it also holds

$$
\begin{array}{ll}
\hat{b}_{\eta}\left(\chi_{0}\right) \rightarrow \hat{b}\left(\chi_{0}\right) & \text { strongly in } L^{1}\left(0, T ; L^{1}(\Omega)\right), \\
\hat{b}_{\eta}\left(\chi_{\eta}\right) \rightarrow \hat{b}(\chi) & \text { strongly in } L^{1}\left(0, T ; L^{1}(\Omega)\right),
\end{array}
$$

for $\eta \rightarrow 0$. Hence, (W2) follows from (W2) $\eta$ for $\eta \rightarrow 0$. 


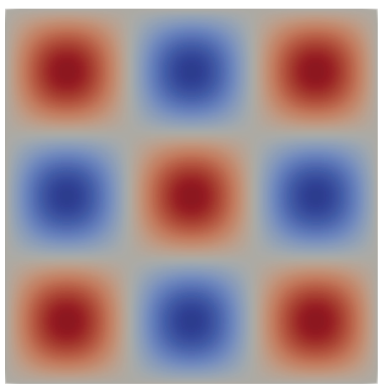

(A)
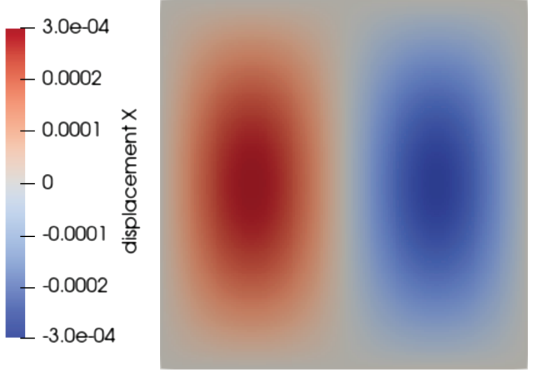

(в)

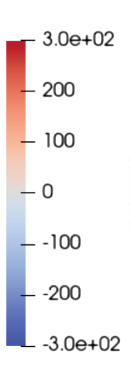

$\underset{\frac{0}{3}}{\frac{0}{2}}$

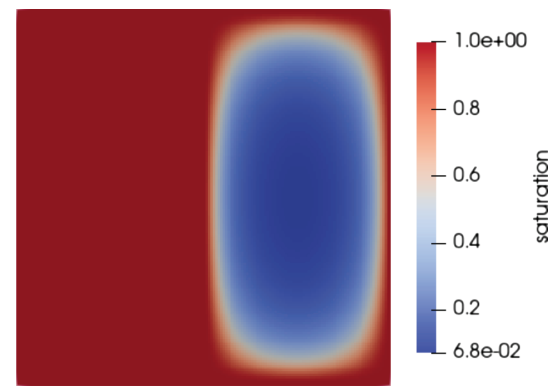

(c)

Figure 2. Solution at time $T=3$, at refinement level 4 , cf., Table 1. (A) $x$-component of $\boldsymbol{u}$. (B) $p_{\mathrm{w}} \cdot(\mathrm{C}) s_{\mathrm{w}}$.

Lemma 9.6 (Initial condition for the volumetric deformation). The volumetric deformation $\boldsymbol{\nabla} \cdot \boldsymbol{u}$ satisfies (W3).

Proof. The proof is almost identical to the proof of Lemma 8.8. Standard compactness arguments and (W3) $\eta$ yield

$$
\int_{0}^{T} a\left(\partial_{t} \boldsymbol{u}, \boldsymbol{v}\right) \mathrm{d} t+\int_{0}^{T} a\left(\boldsymbol{u}-\boldsymbol{u}_{0}, \partial_{t} \boldsymbol{v}\right) \mathrm{d} t=0
$$

for all $\boldsymbol{v} \in H^{1}(0, T ; \boldsymbol{V})$ with $\boldsymbol{v}(T)=\mathbf{0}$. Hence, $\boldsymbol{u}(0)=\boldsymbol{u}_{0}$ in $\boldsymbol{V}$, with $\boldsymbol{u}_{0}$ satisfying (3.1); note that $\boldsymbol{u} \in C(0, T ; \boldsymbol{V})$ by a Sobolev embedding. Therefore also $\boldsymbol{\nabla} \cdot \boldsymbol{u}(0)=\boldsymbol{\nabla} \cdot \boldsymbol{u}_{0}$ in $L^{2}(\Omega)$, which yields (W3).

\subsection{Identifying a weak solution for $\eta \rightarrow 0$}

Finally, we conclude the existence of a weak solution to the unsaturated poroelasticity model.

Lemma 9.7 (Limit satisfies (W1)-(W4)). The limit $(\boldsymbol{u}, \chi)$ is a weak solution of $(2.12)-(2.15)$, cf., Definition 3.1.

Proof. The proof follows directly from the convergence results in Lemmas 9.3-9.6 together with the validity of the regularized problem (4.4) for $\zeta=0$.

\section{NumERICAL TEST}

We illustrate the convergence properties of the numerical scheme introduced in Sections 4 and 5 . For this, we consider a numerical example based on a manufactured solution to the original continuous model (2.1) and (2.2) with corresponding source terms. Subsequently, we solve the regularized and discretized model, and study the convergence towards the analytical solution as the discretization and regularization parameters tend towards zero. In the above analysis, the convergence of the approximations is used to prove the existence of solutions, and no convergence order is provided. Therefore, this order is left aside here as well.

We consider a poroelastic medium with constitutive laws given by the van Genuchten-Mualem relations (2.3) and the equivalent pore pressure (2.4). In particular, let $\Omega=(0,1)^{2}$ denote the medium with the properties $\phi=0.1, c_{\mathrm{w}}=0, \kappa_{\mathrm{abs}}=10^{-8}, N=10^{9}, \alpha=1, \mu=10^{8}, \lambda=10^{7}, \alpha_{\mathrm{vG}}=0.02, n_{\mathrm{vG}}=2.5, s_{\mathrm{w}, \text { res }}=10^{-6}$, $s_{\varepsilon}=0.01-$ the same set of parameters is considered in Section A.

For the original model (2.1) and (2.2), we consider the manufactured solution for $(x, y) \in(0,1)^{2}, t \in[0,3]$

$$
\boldsymbol{u}(x, y, t)=t \cdot 10^{-5}\left[\begin{array}{l}
\sin (2 \pi x) \sin (2 \pi y) \\
\sin (3 \pi x) \sin (3 \pi y)
\end{array}\right], \quad p_{\mathrm{w}}(x, y, t)=t \cdot 10 \sin (2 \pi x) \sin (\pi y)
$$


TABLE 1. Convergence of the (physical) fluid pressure and displacement for consecutively refined spatial and temporal discretization, and regularization.

\begin{tabular}{lllllll}
\hline \hline Level & $h$ & $\tau$ & $\zeta$ & $\eta$ & $\left\|p_{\mathrm{w}}\left(\bar{\chi}_{h \tau}(T)\right)-p_{\mathrm{w}}(T)\right\|_{L^{2}(\Omega)}$ & $\left\|\overline{\boldsymbol{u}}_{h \tau}(T)-\boldsymbol{u}(T)\right\|_{L^{2}(\Omega)}$ \\
\hline 1 & $1 / 20$ & 0.2 & 1 & $1 \mathrm{e}-9$ & $1.70 \mathrm{e} 1$ & $7.12 \mathrm{e}-6$ \\
2 & $1 / 40$ & 0.1 & 0.25 & $0.25 \mathrm{e}-9$ & $8.11 \mathrm{e} 0$ & $1.77 \mathrm{e}-6$ \\
3 & $1 / 80$ & 0.05 & 0.0625 & $0.0625 \mathrm{e}-9$ & $4.01 \mathrm{e} 0$ & $4.42 \mathrm{e}-7$ \\
4 & $1 / 160$ & 0.025 & 0.015625 & $0.015625 \mathrm{e}-9$ & $2.00 \mathrm{e} 0$ & $1.11 \mathrm{e}-7$ \\
\hline
\end{tabular}

with the corresponding transform $\chi$ for $p_{\mathrm{w}}$. The solution combines both saturated and unsaturated zones. The configuration at final time $T=3$ is displayed in Figure 2.

We solve the regularized and discretized approximation of the continuous model, $c f$., Sections 4 and 5 , with source terms corresponding to the manufactured solution of the continuous model (2.1) and (2.2). For this we consider four levels of structured quadrilateral meshes, time partitions, and regularization parameters, as displayed in Table 1. For the discretization of the displacement, we employ piecewise quadratic elements, which, in combination with the finite volume formulation based on piecewise constant functions, are inf-sup stable. We measure the total approximation error - we simply consider the $L^{2}(\Omega)$ errors of the pressure, $p_{\mathrm{w}}$, and displacement, $\boldsymbol{u}$, at the final time $T=3$. The evolution of errors is displayed in Table 1 .

We can observe numerical convergence, consistent with the above analysis. Further study of accuracy properties, as well as existence and uniqueness, of the discretization of the unregularized model are planned in the future.

\section{Appendix A. Practical set of COnstitutive laWs}

In this section, we consider the constitutive laws for $s_{\mathrm{w}}, \kappa_{\text {rel }}$ and $p_{\text {pore }}$, as presented in Section 2.1, and show that in this case that the existence theory presented in this work is applicable. In particular, we consider the van Genuchten-Mualem model (2.3) and the equivalent pore pressure (2.4). Furthermore, we consider a non-degenerate system with $s_{\mathrm{w} \text {,res }}>0$ and $s_{\varepsilon}>0$, ensuring uniformly positive values for the fluid saturation and relative permeability. Finally, we consider $b$ as given in (2.6). In the following, we check all assumptions on the constitutive laws, i.e., both the assumptions (A0)-(A4) and the conditions (ND2) and (ND3). We also check the statement made in Remark 3.2.

Assumption (A0). By definition, it holds $s_{\mathrm{w}}(\mathbb{R})=\left(s_{\mathrm{w}, \mathrm{res}}, 1\right]$ and $\kappa_{\text {rel }}\left(s_{\mathrm{w}}\right)>0$ for any $s_{\mathrm{w}} \in\left(s_{\mathrm{w}, \text { res }}, 1\right]$.

Assumption (A2). By definition, $s_{\mathrm{w}}$ is differentiable with a non-negative and uniformly bounded derivative $s_{\mathrm{w}}^{\prime}$, and it holds that $\hat{s}_{\mathrm{w}}^{\prime}(\chi)=\frac{s_{\mathrm{w}}^{\prime}\left(\hat{p}_{\mathrm{w}}(\chi)\right)}{\hat{\kappa}_{\mathrm{rel}}(\chi)} \geq 0$ for all $\chi \in \mathbb{R}$.

Assumption (A3). By definition, it holds that $p_{\text {pore }}^{\prime}\left(p_{\mathrm{w}}\right)=s_{\mathrm{w}}\left(p_{\mathrm{w}}\right)$ and hence $\hat{p}_{\text {pore }}^{\prime}(\chi)=\frac{\hat{s}_{\mathrm{w}}(\chi)}{\hat{\kappa}_{\mathrm{rel}}(\chi)}$. In addition, for $p_{\mathrm{w}} \geq 0$ it holds that $s_{\mathrm{w}}\left(p_{\mathrm{w}}\right)=1$ and, consequently, $p_{\text {pore }}\left(p_{\mathrm{w}}\right)=p_{\mathrm{w}}$ as well as $\hat{p}_{\text {pore }}(\chi)=\chi$ for $\chi \geq 0$.

Assumption (A1)/(A1) $)_{\eta}$ We consider simultaneously (A1) and (A1) $)_{\eta}$, with $\hat{b}=\hat{b}_{\eta}$ for $\eta=0$. It holds that

$$
\hat{b}_{\eta}^{\prime}(\chi)=\phi_{0} \frac{s_{\mathrm{w}}^{\prime}\left(\hat{p}_{\mathrm{w}}(\chi)\right)}{\hat{\kappa}_{\mathrm{rel}}(\chi)}+c_{\mathrm{w}} \phi_{0} \frac{\hat{s}_{\mathrm{w}}(\chi)}{\hat{\kappa}_{\mathrm{rel}}(\chi)}+\left(\frac{1}{N}+\eta\right) \frac{\hat{s}_{\mathrm{w}}(\chi)^{2}}{\hat{\kappa}_{\mathrm{rel}}(\chi)} \geq 0,
$$

where the positivity results since $\hat{s}_{\mathrm{w}}$ is non-decreasing, and $\hat{s}_{\mathrm{w}}$ and $\hat{\kappa}_{\text {rel }}$ are positive functions. From (App. A.1), it becomes also clear that $\hat{b}_{\eta}^{\prime}$ is uniformly positive if $\eta>0$. In fact, if $c_{\mathrm{w}}>0$ or $\frac{1}{N}>0$, i.e., the fluid or the grains are compressible. The uniform positivity of $\hat{b}_{\eta}^{\prime}$ also follows for $\eta=0, c f$. ., Remark 8.10. Without presenting any calculations, we stress that $(\mathrm{A} 1)_{\eta}$ also follows in the degenerate case $s_{\mathrm{w}, \mathrm{res}}=s_{\varepsilon}=0$. 
Assumption (A4). Using the definition of the equivalent pore pressure (2.4) and the saturation (2.3), it holds that $p_{\text {pore }}^{\prime}\left(p_{\mathrm{w}}\right)=s_{\mathrm{w}}\left(p_{\mathrm{w}}\right) \in[0,1]$. Consequently, for all $p_{\mathrm{w}} \in \mathbb{R}$, one has

$$
\frac{\mathrm{d}}{\mathrm{d} p_{\mathrm{w}}}\left(\frac{p_{\text {pore }}}{s_{\mathrm{w}}}\right)=1-\frac{p_{\text {pore }}\left(p_{\mathrm{w}}\right) s_{\mathrm{w}}^{\prime}\left(p_{\mathrm{w}}\right)}{s_{\mathrm{w}}\left(p_{\mathrm{w}}\right)^{2}} \geq 1
$$

Here, we note that by definition for $p_{\mathrm{w}} \geq 0$ it holds $s_{\mathrm{w}} \equiv 1$ and therefore $s_{\mathrm{w}}^{\prime}\left(p_{\mathrm{w}}\right)=0$, and for $p_{\mathrm{w}}<0$ it holds $s_{\mathrm{w}}^{\prime}\left(p_{\mathrm{w}}\right)>0$ and $p_{\text {pore }}\left(p_{\mathrm{w}}\right)<0$, resulting in the above lower bound. In addition, by the definition of the Kirchhoff transform (2.10) and the fact that $\kappa_{\text {rel }}\left(s_{\mathrm{w}}\right) \in[0,1]$ for all $s_{\mathrm{w}} \in(0,1]$, it holds that $\chi^{\prime}\left(p_{\mathrm{w}}\right)=\kappa_{\text {rel }}\left(s_{\mathrm{w}}\left(p_{\mathrm{w}}\right)\right) \leq 1$. Hence, using the chain rule, $\frac{\hat{p}_{\text {pore }}}{\hat{s}_{\mathrm{w}}}$ satisfies the uniform growth condition (A4) with

$$
\frac{\mathrm{d}}{\mathrm{d} \chi}\left(\frac{\hat{p}_{\text {pore }}}{\hat{s}_{\mathrm{w}}}\right)=\frac{\mathrm{d}}{\mathrm{dp}_{\mathrm{w}}}\left(\frac{p_{\text {pore }}}{s_{\mathrm{w}}}\right) \frac{1}{\chi^{\prime}\left(p_{\mathrm{w}}\right)} \geq 1
$$

Condition (ND2). A simple calculation yields $\hat{p}_{\text {pore }}^{\prime}(\chi)=\frac{\hat{s}_{\mathrm{W}}}{\hat{\kappa}_{\text {rel }}}$, which is bounded from below and above in the non-degenerate case, since $\hat{s}_{\mathrm{w}}$ and $\hat{\kappa}_{\text {rel }}$ are uniformly positive.

Condition (ND3). First, we note that condition (ND3) is automatically satisfied for $\chi \geq 0$ since $\hat{s}_{\mathrm{w}} \equiv \hat{\kappa}_{\text {rel }} \equiv 1$ and thereby $\hat{s}_{\mathrm{w}}(\chi)-\hat{p}_{\text {pore }}^{\prime}(\chi) \equiv 0$. We focus on $\chi<0$. For the equivalent pore pressure with $\hat{p}_{\text {pore }}^{\prime}=\frac{\hat{s}_{\mathrm{w}}}{\hat{\kappa}_{\mathrm{rel}}}$, the condition (ND3) equivalently reads (the arguments are suppressed)

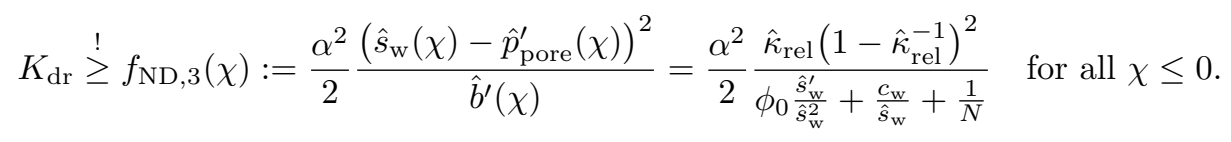

If the bulk is sufficiently stiff (meaning that $K_{\mathrm{dr}}$ is sufficiently large), and the system is just slightly compressible, i.e., $c_{\mathrm{w}}$ and/or $\frac{1}{N}$ are positive, there exists a regularization parameter $s_{\varepsilon}>0$ such that (ND3) is satisfied.

In this sense, we consider a realistic example material, which is relevant in the context of unsaturated poroelasticity: a sandy clay loam saturated with water. Typical values $[16,44]$ of the model parameters are given by $\alpha_{\mathrm{vG}}=0.02, n_{\mathrm{vG}}=2.5, \phi_{0}=0.4, \alpha=1, N=10^{9} \mathrm{~Pa}, c_{\mathrm{w}}=10^{-10} \mathrm{~Pa}^{-1}$. We plot the right hand side expression of (App. A.2) for different regularization parameters, $s_{\varepsilon} \in\{0.01,0.05,0.1\}$, and residual saturations, $s_{\mathrm{w}, \text { res }} \in\left\{10^{-6}, 10^{-2}\right\}, c f$., Figure A.1. We observe, that for each scenario, the right hand side expression of (App. A.2) is uniformly bounded. Hence, if $K_{\mathrm{dr}}$ is larger than this bound, (ND3) holds. Comparing with the realistic value $K_{\mathrm{dr}} \sim 10^{8} \mathrm{~Pa}$, we conclude that the condition (ND3) can be indeed expected to be satisfied for certain materials in geotechnical applications.

Discussion of (W1). Assume $\chi \in L^{2}\left(Q_{T}\right)$. By the definition of $s_{\mathrm{w}}, \hat{s}_{\mathrm{W}}(\chi)$ has values in [0,1] a.e., and therefore $\hat{s}_{\mathrm{w}}(\chi) \in L^{\infty}\left(Q_{T}\right)$. Furthermore, one has $\hat{p}_{\text {pore }}(\chi)=\int_{0}^{\hat{p}_{\mathrm{w}}(\chi)} s_{\mathrm{w}}(q) d q=\int_{0}^{\chi} \frac{\hat{s}_{\mathrm{w}}(x)}{\hat{\kappa}_{\mathrm{rel}}(x)} \mathrm{d} x$. Hence, assuming $s_{\varepsilon}>0$, it holds that $\kappa_{\text {rel }}$ is uniformly bounded from below, and $\left\|\hat{p}_{\text {pore }}(\chi)\right\|_{L^{2}\left(Q_{T}\right)} \leq\left\|\frac{s_{\mathrm{W}}}{\kappa_{\mathrm{rel}}}\right\|_{L^{\infty}\left(Q_{T}\right)}\|\chi\|_{L^{2}\left(Q_{T}\right)}<\infty$. This gives $\hat{p}_{\text {pore }}(\chi) \in L^{2}\left(Q_{T}\right)$.

\section{Appendix B. Useful Results From literature}

Lemma B.1 (Discrete Poincaré inequality [19]). Let $\mathcal{T}$ be an admissible mesh, cf., Definition 5.1, and u a piecewise constant, scalar function. Then there exists a constant $C_{\Omega, \mathrm{DP}} \in(0, \operatorname{diam}(\Omega)]$ such that

$$
\|u\|_{L^{2}(\Omega)} \leq C_{\Omega, \mathrm{DP}}\|u\|_{1, \mathcal{T}},
$$

where $\|\cdot\|_{1, \mathcal{T}}$ denotes the discrete $H_{0}^{1}(\Omega)$ norm, introduced in Definition 5.3. 


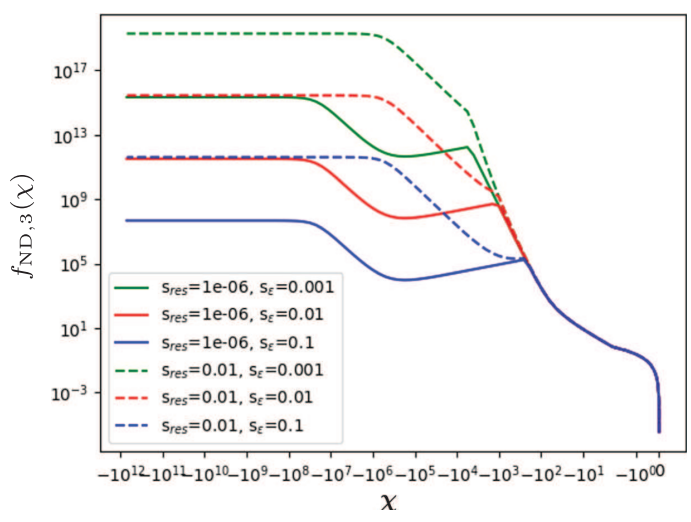

Figure A.1. The dependence of $f_{\mathrm{ND}, 3}$ from (App. A.2), for various values of $s_{\mathrm{w}, \text { res }}$ and $s_{\varepsilon}$.

Lemma B.2 (Discrete trace inequality [20]). Let $\mathcal{T}$ be an admissible mesh, cf., Definition 5.1, and u a piecewise constant function. Let $\gamma(u)$ denote the restriction of $u$ onto the boundary, defined by $\gamma(u)=u_{K}$ on $\sigma \in \mathcal{E}_{\text {ext }} \cap \mathcal{E}_{K}$, $K \in \mathcal{T}$. Then there exists a constant $C_{\mathrm{tr}}>0$ such that

$$
\|\gamma(u)\|_{L^{2}(\partial \Omega)} \leq C_{\operatorname{tr}}\left(\|u\|_{1, \mathcal{T}}+\|u\|_{L^{2}(\Omega)}\right),
$$

where $\|\cdot\|_{1, \mathcal{T}}$ denotes the discrete $H_{0}^{1}(\Omega)$ norm, introduced in Definition 5.3.

Lemma B.3 (Stability of discrete gradients [19]). Let $\mathcal{T}$ be an admissible mesh of some domain $\Omega$, cf., Definition 5.1, and $u \in H_{0}^{1}(\Omega)$. Define a piecewise constant function $\tilde{u}$ by

$$
\tilde{u}(x):=\frac{1}{|K|} \int_{K} u(x) \mathrm{d} x, \quad x \in K \in \mathcal{T} .
$$

Then there exists a constant $C>0$ (independent of $h$ for regular meshes) such that

$$
\|\tilde{u}\|_{1, \mathcal{T}} \leq C\|u\|_{H^{1}(\Omega)} .
$$

Lemma B.4 (Corollary of Brouwer's fixed point theorem [14]). Let $\langle\cdot, \cdot\rangle$ denote the standard $\mathbb{R}^{d}$ scalar product and let $\boldsymbol{F}: \mathbb{R}^{d} \rightarrow \mathbb{R}^{d}$ be a continuous function, satisfying

$$
\langle\boldsymbol{F}(\boldsymbol{x}), \boldsymbol{x}\rangle \geq 0
$$

for all $\boldsymbol{x} \in \mathbb{R}^{d}$ with $\langle\boldsymbol{x}, \boldsymbol{x}\rangle \geq M$ for some fixed $M \in \mathbb{R}_{+}$. Then there exists a $\boldsymbol{x}^{\star} \in \mathbb{R}^{d}$ with $\left\langle\boldsymbol{x}^{\star}, \boldsymbol{x}^{\star}\right\rangle \leq M$ and $\boldsymbol{F}\left(\boldsymbol{x}^{\star}\right)=0$.

Lemma B.5 (Binomial identity). For $a, b \in \mathbb{R}$ it holds that

$$
a(a-b)=\frac{1}{2}\left(a^{2}+(a-b)^{2}-b^{2}\right) .
$$

Lemma B.6 (Summation by parts). Given two sequences $\left(a_{k}\right)_{k \in \mathbb{N}_{0}},\left(b_{k}\right)_{k \in \mathbb{N}_{0}} \subset \mathbb{R}$, for all $N \in \mathbb{N}$ it holds that

$$
\sum_{n=1}^{N} a_{n}\left(b_{n}-b_{n-1}\right)=a_{N} b_{N}-a_{1} b_{0}-\sum_{n=1}^{N-1} b_{n}\left(a_{n+1}-a_{n}\right) .
$$


Lemma B.7 (Discrete Grönwall inequality [15]). Let $\left(a_{n}\right)_{n} \subset \mathbb{R}_{+},\left(\lambda_{n}\right)_{n} \subset \mathbb{R}_{+}, B \geq 0$. Assume for all $n \in \mathbb{N}$ it holds that $a_{n} \leq B+\sum_{k=0}^{n-1} \lambda_{k} a_{k}$. Then it follows $a_{n} \leq B \prod_{k=0}^{n-1}\left(1+\lambda_{k}\right)$. In particular, if $\lambda_{k}=\frac{\lambda T}{N}$ for all $k \in \mathbb{N}$ for some $\lambda, T \in \mathbb{R}_{+}$and $N \in \mathbb{N}$, it holds that $a_{N} \leq B \exp (\lambda T)$.

Lemma B.8 (Eberlein-Šmulian theorem [14]). Assume that $B$ is a reflexive Banach space and let $\left\{x_{n}\right\}_{n} \subset B$ be a bounded sequence in $B$. Then there exists a subsequence $\left\{x_{n_{k}}\right\}_{k}$ that converges weakly in $B$.

Lemma B.9 (Relaxed Aubin-Lions lemma [38]). Let $\left\{f_{n}\right\}_{n} \subset L^{p}(0, T ; B), 1 \leq p<\infty, B$ a Banach space. $\left\{f_{n}\right\}_{n}$ is relatively compact in $L^{p}(0, T ; B)$ if the following two are fulfilled:

- $\left\{f_{n}\right\}_{n}$ is uniformly bounded in $L^{p}(0, T ; X)$, for $X \subset B$ with compact embedding.

- $\int_{\tau}^{T}\left\|f_{n}(t)-f_{n}(t-\tau)\right\|_{B}^{p} \mathrm{~d} t \leq \mathcal{O}(\tau)$, as $\tau \rightarrow 0$.

For the second property it is sufficient that $\left\{\partial_{t} f_{n}\right\}_{n}$ is uniformly bounded in $L^{p}(0, T ; B)$.

Lemma B.10 (Riesz-Frechet-Kolmogorov compactness criterion [10]). Let $F$ be a bounded set in $L^{p}\left(\mathbb{R}^{N}\right)$ with $1 \leq p<\infty, N \in \mathbb{N}$. Assume that

$$
\lim _{|h| \rightarrow 0}\|f(\cdot+h)-f(\cdot)\|_{L^{p}\left(\mathbb{R}^{N}\right)}=0 \quad \text { uniformly in } f \in F .
$$

Then the closure of $\left.F\right|_{\Omega}:=\{f: \Omega \rightarrow \mathbb{R} \mid f \in F\}$ is compact for any measurable set $\Omega \subset \mathbb{R}^{N}$ with finite measure.

Lemma B.11 (Standard inf-sup argument [6]). Let $V$ and $Q$ be Hilbert spaces, and let $B$ be a linear continuous operator from $V$ to $Q^{\prime}$. Denote by $B^{t}$ the transposed operator of $B$. Then, the following two statements are equivalent:

- $B^{t}$ is bounding, i.e., there exists a $\gamma>0$ such that $\left\|B^{t} q\right\|_{V^{\prime}} \geq \gamma\|q\|_{Q}$ for all $q \in Q$.

- There exists a $L_{B} \in \mathcal{L}\left(Q^{\prime}, V\right)$ such that $B\left(L_{B}(\xi)\right)=\xi$ for all $\xi \in Q^{\prime}$ with $\left\|L_{b}\right\|=\frac{1}{\gamma}=: C_{\Omega \text {,is }}$.

Lemma B.12 (Properties of energies based on the Legendre transformation [1]). Given $b: \mathbb{R} \rightarrow \mathbb{R}$ continuous and non-decreasing, i.e., with a differentiable convex potential $\varphi: \mathbb{R} \rightarrow \mathbb{R}$, we define the energy $B$ via the Legendre transformation of $\varphi$ evaluated in $b$

It holds for all $x, y \in \mathbb{R}$ and for all $\mathcal{F}^{\mathbb{R}}>0$

$$
\begin{aligned}
& B(z):=\sup _{\text {and for } a \ell \mathcal{R}^{\mathbb{R}}>0}(b(z) \sigma-\varphi(\sigma)+\varphi(0))=\int_{0}^{z}(b(z)-b(s)) \mathrm{d} s \geq 0 . \\
& \text { and }
\end{aligned}
$$

$$
\begin{aligned}
0 & \leq B(x), \\
B(x)-B(y) & \leq(b(x)-b(y)) x, \\
|b(x)| & \leq \delta B(x)+\sup _{|y| \leq \delta^{-1}}|b(y)| .
\end{aligned}
$$

Acknowledgements. JWB was supported in part by the Meltzer Research Fund, Research Council of Norway (RCN) Project 250223, and the FracFlow project funded by Equinor, Norway, through Akademiaavtalen. ISP was supported by the Research Foundation-Flanders (FWO), Belgium through the Odysseus programme (project G0G1316N) and the Akademia grant of Equinor. IY was supported in part by NSF grant DMS 1818775, NSF grant DMS 2111129, and Oberwolfach Simons Visiting Professorship. In addition, the authors would like to thank Clément Cancès, Willi Jäger, Florin A. Radu, and Hamdi Tchelepi for their useful comments. Furthermore, the authors appreciate the comments of the reviewers which significantly improved the manuscript. 


\section{REFERENCES}

[1] H.W. Alt and S. Luckhaus, Quasilinear elliptic-parabolic differential equations. Math. Z. 183 (1983) 311-341.

[2] I. Ambartsumyan, V.J. Ervin, T. Nguyen and I. Yotov, A nonlinear Stokes-Biot model for the interaction of a non-Newtonian fluid with poroelastic media. ESAIM: M2AN 53 (2019) 1915-1955.

[3] J.-L. Auriault and E. Sanchez-Palencia, Etude de comportment macroscopique d'un milieu poreux sature deformable. J. de Méc. 16 (1977) 575-603.

[4] M. Biot, General theory of three-dimensional consolidation. J. Appl. Phys. 12 (1941) 155-164.

[5] L. Bociu, G. Guidoboni, R. Sacco and J.T. Webster, Analysis of nonlinear poro-elastic and poro-visco-elastic models. Arch. Ration. Mech. Anal. 222 (2016) 1445-1519.

[6] D. Boffi, F. Brezzi and M. Fortin, Mixed Finite Element Methods and Applications. Springer Series in Computational Mathematics. Springer (2013).

[7] F. Bonaldi, K. Brenner, J. Droniou and R. Masson, Gradient discretization of two-phase flows coupled with mechanical deformation in fractured porous media. Comput. Math. App. 98 (2021) 40-68.

[8] J.W. Both, K. Kumar, J.M. Nordbotten and F.A. Radu, Anderson accelerated fixed-stress splitting schemes for consolidation of unsaturated porous media. Comput. Math. App. 77 (2019) 1479-1502.

[9] J.W. Both, K. Kumar, J.M. Nordbotten and F.A. Radu, The gradient flow structures of thermo-poro-visco-elastic processes in porous media. Preprint: arXiv:1907.03134 (2019).

[10] H. Brezis, Functional Analysis, Sobolev Spaces and Partial Differential Equations. Springer Science \& Business Media (2010).

[11] M.K. Brun, E. Ahmed, J.M. Nordbotten and F.A. Radu, Well-posedness of the fully coupled quasi-static thermo-poroelastic equations with nonlinear convective transport. J. Math. Anal. App. 471 (2019) 239-266.

[12] Q.M. Bui, D. Osei-Kuffuor, N. Castelletto and J.A. White, A scalable multigrid reduction framework for multiphase poromechanics of heterogeneous media. SIAM J. Sci. Comput. 42 (2020) B379-B396.

[13] J.T. Camargo, J.A. White and R.I. Borja, A macroelement stabilization for multiphase poromechanics. Comput. Geosci. 25 (2021) 775-792.

[14] P.G. Ciarlet, Linear and Nonlinear Functional Analysis with Applications. Society for Industrial and Applied Mathematics, Philadelphia, PA, USA (2013).

[15] D.S. Clark, Short proof of a discrete Gronwall inequality. Discrete Appl. Math. 16 (1987) 279-281.

[16] O. Coussy, Poromechanics. Wiley (2004).

[17] R. de Boer, Theory of Porous Media: Highlights in Historical Development and Current State. Springer Science \& Business Media (2000).

[18] L. Evans, Partial Differential Equations: Graduate Studies in Mathematics. American Mathematical Society (2010).

[19] R. Eymard, T. Gallouët and R. Herbin, Convergence of finite volume schemes for semilinear convection diffusion equations. Numer. Math. 82 (1999) 91-116.

[20] R. Eymard, T. Gallouët and R. Herbin, Finite volume methods. Handb. Numer. Anal. 7 (2000) $713-1018$.

[21] R. Eymard, R. Herbin and A. Michel, Mathematical study of a petroleum-engineering scheme. ESAIM: M2AN 37 (2003) 937-972.

[22] F. Gaspar, J. Gracia, F. Lisbona and P. Vabishchevich, A stabilized method for a secondary consolidation Biot's model. Numer. Methods Part. Differ. Equ. 24 (2008) 60-78.

[23] R. Lewis and B. Schrefler, The finite element method in the static and dynamic deformation and consolidation of porous media. In: Numerical Methods in Engineering. John Wiley (1998).

[24] A. Mikelić and M.F. Wheeler, Theory of the dynamic Biot-Allard equations and their link to the quasi-static Biot system. J. Math. Phys. 53 (2012) 123702.

[25] A. Mikelić and M.F. Wheeler, Convergence of iterative coupling for coupled flow and geomechanics. Comput. Geosci. 17 (2013) $455-461$.

[26] A. Mikelić, M.F. Wheeler and T. Wick, Phase-field modeling of a fluid-driven fracture in a poroelastic medium. Comput. Geosci. 19 (2015) 1171-1195.

[27] Y. Mualem, A new model for predicting the hydraulic conductivity of unsaturated porous media. Water Res. Res. 12 (1976) $513-522$.

[28] M.A. Murad and J.H. Cushman, Multiscale flow and deformation in hydrophilic swelling porous media. Int. J. Eng. Sci. 34 (1996) 313-338.

[29] R.H. Nochetto and C. Verdi, Approximation of degenerate parabolic problems using numerical integration. SIAM J. Numer. Anal. 25 (1988) 784-814.

[30] J.M. Nordbotten and M.A. Celia, Geological Storage of $\mathrm{CO}_{2}$ : Modeling Approaches for Large-scale Simulation. John Wiley \& Sons (2011).

[31] I.S. Pop and B. Schweizer, Regularization schemes for degenerate Richards equations and outflow conditions. Math. Models Methods Appl. Sci. 21 (2011) 1685-1712.

[32] C. Rodrigo, X. Hu, P. Ohm, J.H. Adler, F.J. Gaspar and L.T. Zikatanov, New stabilized discretizations for poroelasticity and the Stokes' equations. Comput. Methods Appl. Mech. Eng. 341 (2018) 467-484.

[33] M.E. Rose, Numerical methods for flows through porous media. I. Math. Comp. 40 (1983) 435-467.

[34] B. Saad and M. Saad, Study of fully implicit petroleum engineering finite-volume scheme for compressible two-phase flow in porous media. SIAM J. Numer. Anal. 51 (2013) 716-741. 
[35] R. Showalter, Diffusion in poro-elastic media. J. Math. Anal. App. 251 (2000) 310-340.

[36] R.E. Showalter, Poroelastic filtration coupled to Stokes flow. Lecture Notes Pure Appl. Math. 242 (2005) 229.

[37] R. Showalter and N. Su, Partially saturated flow in a poroelastic medium. Discrete Continuous Dyn. Syst. Ser. B 1 (2001) 403-420.

[38] J. Simon, Compact sets in the space $L^{p}(0, T ; B)$. Ann. Mat. Pura App. 146 (1986) 65-96.

[39] A. Szymkiewicz, Modelling Water Flow in Unsaturated Porous Media: Accounting for Nonlinear Permeability and Material Heterogeneity. Springer Science \& Business Media (2012).

[40] A. Tavakoli and M. Ferronato, On existence-uniqueness of the solution in a nonlinear Biot's model. Appl. Math 7 (2013) $333-341$.

[41] K.V. Terzaghi, The shearing resistance of saturated soils and the angle between the planes of shear. In: First International Conference on Soil Mechanics. 1 (1936) 54-59.

[42] C.J. van Duijn and A. Mikelić, Mathematical Theory of Nonlinear Single-Phase Poroelasticity (2019) https://hal-univ-lyon1. archives-ouvertes.fr/hal-02144933.

[43] C. van Duijn, A. Mikelić, M.F. Wheeler and T. Wick, Thermoporoelasticity via homogenization: modeling and formal two-scale expansions. Int. J. Eng. Sci. 138 (2019) 1-25.

[44] M. van Genuchten, A closed-form equation for predicting the hydraulic conductivity of unsaturated soils. Soil Sci. Soc. Am. J. 44 (1980) 892-898.

[45] M. Wheeler, G. Xue and I. Yotov, Coupling multipoint flux mixed finite element methods with continuous Galerkin methods for poroelasticity. Comput. Geosci. 18 (2014) 57-75.

[46] J.A. White, N. Castelletto, S. Klevtsov, Q.M. Bui, D. Osei-Kuffuor and H.A. Tchelepi, A two-stage preconditioner for multiphase poromechanics in reservoir simulation. Comput. Methods Appl. Mech. Eng. 357 (2019) 112575.

[47] A. Zenisek, The existence and uniquencess theorem in Biot's consolidation theory. Aplikace Matematiky 29 (1984) $194-211$.

\section{Subscribe to Open (S2O)}

\section{A fair and sustainable open access model}

This journal is currently published in open access under a Subscribe-to-Open model (S2O). S2O is a transformative model that aims to move subscription journals to open access. Open access is the free, immediate, online availability of research articles combined with the rights to use these articles fully in the digital environment. We are thankful to our subscribers and sponsors for making it possible to publish this journal in open access, free of charge for authors.

\section{Please help to maintain this journal in open access!}

Check that your library subscribes to the journal, or make a personal donation to the S2O programme, by contacting subscribers@edpsciences.org

More information, including a list of sponsors and a financial transparency report, available at: https://www. edpsciences.org/en/maths-s2o-programme 